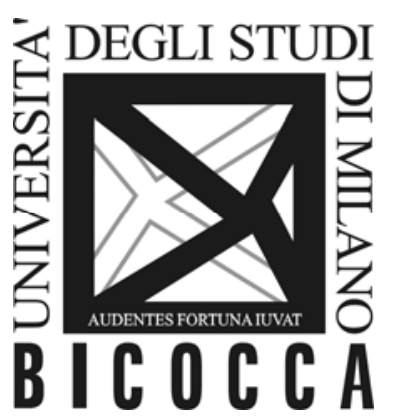

DEPARTMENT OF ECONOMICS,
MANAGEMENT AND STATISTICS
UNIVERSITY OF MILAN - BICOCCA

DEMS WORKING PAPER SERIES

\title{
Logrolling under Fragmented Authoritarianism: Theory and Evidence from China
}

Mario Gilli, Yuan Li, Jiwei Qian

No. 333 - April 2016

Dipartimento di Economia, Metodi Quantitativi e Strategie di Impresa Università degli Studi di Milano - Bicocca http://dems.unimib.it/ 


\title{
Logrolling under Fragmented Authoritarianism: Theory and Evidence from China
}

\author{
Mario Gilli \\ Department of Economics, Management and Statistics. \\ University of Milan-Bicocca. \\ Piazza dell'Ateneo Nuovo,1, Milan, Italy. \\ mario.gilli@unimib.it \\ Yuan Li \\ Mercator School of Management and Institute of East Asian Studies, \\ University of Duisburg-Essen. \\ Forsthausweg 2, Duisburg, Germany. \\ yuan.li@uni-due.de

\section{Jiwei Qian} \\ East Asian Institute, \\ National University of Singapore. \\ 469 Bukit Timah Road, Singapore. \\ jiwei.qian@nus.edu.sg
}

April 3, 2016

\begin{abstract}
Autocratic policy-making processes have been under studied both theoretically and empirically, while most literature on autocracies has assumed them to have a monolithic and top-down nature. This paper seeks to remedy this deficiency by focusing on logrolling among interest groups in fragmented autocracies, and tries to pick apart the black-box of decision making in autocracies. In particular, we focus on China where decision making exemplifies a process of logrolling among key actors in the political system. The key question in this paper is: what are the effects of the logrolling of parochial interest groups on state policies and social welfare in autocracies?
\end{abstract}


We address this question both theoretically and empirically. The theoretical model helps us to focus on a specific distortion in resource allocation because of logrolling, while the econometric results confirm our theoretical prediction in a very robust way. To find out the distinctive consequences of autocratic logrolling on state policy and social welfare, we compare the policy outcomes under logrolling with the policy outcomes under different decision-making rules. We find policy outcomes under logrolling are characterized by excessive spending on all the interest groups' preferred goods and insufficient spending on public goods. Finally, the paper provides empirical evidence on autocratic logrolling by studying the effects of the interaction between two vertical bureaucracies in China - the Ministry of Civil Affairs and the Ministry of Health. In particular, we test the predictions of our model and identify the effects of logrolling in autocracies. Building on the existing literature, we introduce new approaches, including natural experiment and placebo test, to empirically test the existence of logrolling and identify its effects. We show that the direction of inefficiency on resource allocation is in accordance with the prediction in our theoretical model.

Key Words: Authoritarianism, Policy Making, Logrolling, Fragmented Authoritarianism, China.

JEL Codes: D72, H11, H41, P48.

First draft September, 2014

This draft March, $2016^{1}$

"The Chinese government makes policy according to a decision rule of delegation by consensus ...... If the agents reach consensus, the decision is automatically ratified by the higher level; if the agents cannot agree, then the authorities step in to make the decision..."— Shirk 1993, p.116

\footnotetext{
${ }^{1}$ A first draft of this paper was written while Li Yuan was visiting the Department of Economics, Management, and Statistics at Bicocca Univeristy. The hospitality and stimulating working environment are gratefully acknowledged. It was completed a year and half later when Mario Gilli was visiting the Institute of East Asian Studies at the University of Duisburg, whose generous hospitality was crucial to complete the work. We would like to thank Susan Shirk, Chongen Bai,Loren Brandt,Martin Gregor,Peter Lorentzen, Gert Tinggaard Svendsen,Athar Hussain, Pinghan Liang for their comments and insights. We also thank the participants at the First USCD-Tsinghua Conference on China's Political Economy, European Public Choice Society Annual Meeting 2015, the 19th Annual Conference of the International Society for New Institutional Economics, and the 2nd Biennial Conference of China Development Studies, the 2015 Chinese Economic Association (Europe/UK) Annual Conference, the 30th annual congress of the European Economic Association, seminars at Fudan University, Nanjing University, Nanjing University of Science and Technology, Southwestern University of Finance and Economics for helpful discussions.
} 


\section{Introduction}

It might seem that policy-making processes in autocracies are simply monolithic and top-down, because policy decisions are taken according to a cabal of key leaders' wishes that is then implemented by a subservient bureaucracy. However, a closer scrutiny of the actual operation of policy-making in autocracies shows that different groups inside the autocratic political system pursue their own interests using their own strategic power, and it is their strategic interactions that give rise to state policies. But these complex strategic interactions and their consequences on policy-making in autocracies has not previously been taken seriously enough.

This paper seeks to remedy this deficiency by focusing on logrolling among these interest groups in fragmented autocracies, and tries to pick apart the blackbox of decision-making in autocracies. In fact, the logrolling of parochial interest groups is one of the most important features of decision-making in all autocratic polities. For instance, the policies in Germany before the First World War were influenced by the logrolling of two parochial interest groups — the "marriage of iron and rye" - in which aristocratic landowners supported a fleet-building program that industrial interests desired, and in exchange, big business supported high agriculture tariffs (Snyder 1991). In this paper, we focus on China, where bureaucratic interest groups are separate vertical organizations reaching down from Beijing to the provinces and cities that operate with little discipline or coordination from above. Each bureaucracy pushes for policies in their own interests - to increase its own budgets, staffing, etc. (Shirk, 2014). They make decisions following the rule, "I will go along with what you want to do in your domain, if you let me call the shots in my domain". ${ }^{2}$

This view leads to the key question of this paper: what are the effects of the logrolling of parochial interest groups on state policies and social welfare in autocracies? The issue of whether logrolling undermines social welfare or produces inefficient policies is a long-standing debate within in the literature (Buchanan and Tullock 1962, Riker and Brahms, 1973, Miller, 1977), but no one has addressed this problem in relation to autocratic systems. In this paper, we address this question both theoretically and empirically. The theoretical model helps us to understand the distortion in resource allocation due to logrolling, while the econometric results confirm our theoretical predictions in a very robust way.

We build a model of autocratic logrolling as a simplified variant of the legislative bargaining models in democracies (e.g. Austen-Smith and Banks 1988; Baron and Ferejohn 1989; Alesina and Rosenthal 1996). However, our model differs from the legislative bargaining models in democracies in the following aspects. First, in the legislative bargaining model, no agreement can be imposed on any individual

\footnotetext{
${ }^{2}$ Interview with Susan Shirk at 2012 .
} 
without his approval, but in our model a default outcome may be imposed by the dictator. Second, the legislative bargaining model is a game where players cannot trade favors with each other, but in our model strategic collaborations of the players are possible. Third, in the legislative bargaining model, all the proposal power is allocated to the proponent, while in our model the proposal power is symmetrically distributed among the players, even if there is a sequential structure. In order to find out the distinctive consequences of autocratic logrolling on state policy and social welfare, we compare the policy outcomes under logrolling with the policy outcomes under different decision-making rules, including decisions made by a utilitarian social planner, by only one interest group, and by a simplified version of legislative bargaining. Our most important finding from the theoretical analysis is that the policy outcome under autocratic logrolling is characterized by excessive spendings on all the interest groups' preferred goods, and in particular, the inefficiency of the resource allocation caused by autocratic logrolling is more serious than by other decision-making rules.

The empirical literature on logrolling is not that rich, probably because the proof of the existence of logrolling depends on the record of the trading of favors, which may not exist (Evans 1994) or may be difficult to acquire (Buchanan and Tullock 1962). The exceptional breakthroughs were made by Stratmann (1992, 1995) who found evidences of logrolling in US congressional voting in the 1960s and 1980s. Later, Irwin and Kroszner (1996), Crombez (2000) and Copelovitch (2010) provided indirect evidences of how interest groups logrolling with each other in determining the US tariff act, EU policies, and IMF lending. Given all the difficulties, our paper contributes to the literature by providing indirect empirical evidences of autocratic logrolling by studying the effects of the interaction between two vertical bureaucracies in China - the Ministry of Civil Affairs (MCA) and the Ministry of Health $(\mathrm{MOH})$. In particular, we test the predictions from our theoretical model and identify the effects of logrolling. Building on Stratmann (1995), we introduce new approaches, including natural experiment and placebo test, to identify the causality of logrolling. We show that resource allocation on the interest groups' most preferred goods tend to be inefficiently high and on public goods tends to be inefficiently low.

The paper proceeds as follows. Section 2 presents the structure of logrolling in China by a case study on logrolling between MCA and MOH. Section 3 provides the theoretical model of logrolling, and comparing its structure with a similar bargaining situation. Section 4 presents the empirical evidence on logrolling between $\mathrm{MCA}$ and $\mathrm{MOH}$. Conclusions are then drawn in the final section, while calculations are presented in the Appendix. 


\section{Some stylized facts on logrolling between MCA and MOH in China}

Both the Ministry of Civil Affairs (MCA) and the Ministry of Health (MOH) are ministries under the jurisdiction of the State Council of China ${ }^{3}$. The MCA is responsible for social and administrative affairs, while the $\mathrm{MOH}$ is responsible for ensuring the accessibility of health services. The two ministries have different priorities on many different issues, however, sometimes they need the other ministry's support to ensure the smooth implementation of their own priority issues, which makes logrolling between them possible.

A typical logrolling between MCA and MOH involves three policies: Dibao (or "Minimum Livelihood Guarantee Scheme"), Rural health insurance (or "New Cooperative Medical Scheme"), and Mental Health Care. Dibao was initiated in the 1990s to provide a safety net for the urban poor. By the end of 2002, over 19 million people were included in the "Dibao" program. A total of 9.8 million former SOE employees and 5.5 million of their family members accounted for about $80 \%$ of total beneficiaries ${ }^{4}$. Dibao is managed by MCA and hence is the core responsibility of MCA. It's objective is to provide as much financial and other assistance programs as possible for households tagged as "Dibao" households. Rural health insurance was initiated in 2004, and is the core responsibility of $\mathrm{MOH}^{5}$ It's objective is to expand the coverage of rural health insurance, and the ideal position for $\mathrm{MOH}$ is to achieve universal coverage. Both ministries are responsible for providing mental healthcare. However, Mental healthcare, although an important public health issue, is not the core responsibility for either MCA or MOH. Nonetheless, both ministries have duties to operate mental hospitals.

As stated by the quotation at the beginning of the article, the Chinese government makes policy according to a decision rule of "delegation by consensus" (Shirk, 1993). If both MCA and MOH stick to their own preferences, they cannot reach consensus. Then, the issues will be either "tabled" or referred to a higher level authority, e.g. the premier or vice premier of the State Council, for resolution $^{6}$. Alternatively, the two ministries might exchange favors regarding each other's prior policy at the expense of the policy that is not essential for either of

\footnotetext{
${ }^{3}$ In the reforms of 2013 the MOH has been dissolved and its functions integrated into the new agency called the National Health and Family Planning Commission.

${ }^{4}$ See China Association of Social Workers (2010).

${ }^{5}$ Both Dibao and Rural health insurance require that a household rather than an individual as a basic statistical unit.

${ }^{6}$ Note that the Ministry of Finance is not likely to be involved in managing these social programs, even though it makes decisions about the allocation of budget. This is because the function of each government department in the post-Mao era has been increasingly differentiated and professional knowledge is required for policy making (Huang 2013: 10).
} 


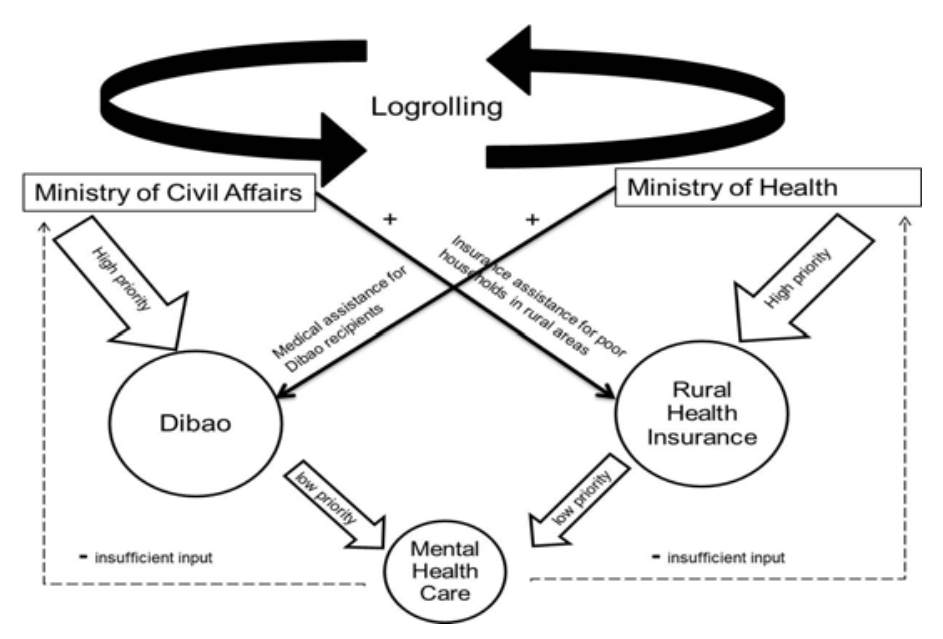

Figure 1: Logrolling between MCA and $\mathrm{MOH}$

them. In reality, MOH supports the Dibao program by allowing Dibao recipients to be automatically eligible for subsidies and sometimes free healthcare services in urban hospitals under the urban medical assistance programs ${ }^{7}$. In exchange, MCA pays the insurance premium for poor households 8 in rural areas from the medical assistance fund that it manages. However, in contrast, mental health care which is not a priority for either of the two ministries, receives insufficient input. Figure ?? shows how $\mathrm{MOH}$ and MCA exchange favors.

Logrolling agreements may be problematic if there is no external institution to guarantee the enforcement of the agreement (Shirk 1993, p.127). With regard to this strategic question, it is essential to know the institutional arrangements for ministries to exchange their support. Enelow (1986) argues that the expectation that logrolling will take place stabilizes the logrolling process. Weingast and Marshal (1988) demonstrate that the committee system in congress serves as a means of enforcement. In China the enforcing of logrolling deals takes the form of inter-ministerial joint conferences (Lianxi Huiyi) among representatives from different ministries. Joint conferences have been held regularly for Dibao, rural health insurance, and mental health care among $\mathrm{MOH}, \mathrm{MCA}$ and other different ministries since $2003^{9}$. Moreover, some of the future logrolling deals are institu-

\footnotetext{
${ }^{7}$ The free treatments Dibao recipients receive include a basic package of services and drugs according to the decision of $\mathrm{MOH}$.

${ }^{8}$ Note that these poor households are defined as poor households who have difficulties to afford medical fees and these poor households are not necessary to be dibao recipients.

${ }^{9}$ For rural health insurance, eleven ministries including $\mathrm{MOH}$ and $\mathrm{MCA}$ have been involved. Representatives from MCA and $\mathrm{MOH}$ are appointed as deputy coordinators of this joint conference. For medical assistance program, MOH, MCA and other ministries hold regular working
} 
tionalized through policy documents that are planned many years in advance. For example, in the guideline for social assistance released by the Chinese government in May 2014, the role and responsibility of different government departments are stated explicitly for the forthcoming expansion of social assistance programs.

What are the consequences in resource allocation due to logrolling between the two ministries? The following descriptive analysis shows the inefficient policy outcomes regarding Dibao, Rural health insurance, and Mental Health Care. The internal logic and causality of between the inefficiencies and logrolling will be studied by a theoretical model with the support of rigorous empirical evidences in later sections.

First, the resource allocation in Dibao is inefficiently too high such that it even crowds out unemployment insurance. After MCA and $\mathrm{MOH}$ exchanging their supports, a Dibao recipient is also entitled to claim benefits from the $\mathrm{MOH}$-supported complementary social assistance programs (e.g. urban medical assistance program). Thus, while the direct benefit level of Dibao itself is modest enough ${ }^{10}$ not to crowd out unemployment insurance, the aggregate benefit from Dibao (including the benefits from the urban medial assistance program and other related programs) may be higher than the benefit from unemployment insurance ${ }^{11}$. Therefore, many people opt out of the unemployment insurance but enroll with Dibao. One stylized fact is that there are much larger urban labor forces enrolled in another social insurance program - the Basic Pension Scheme (BPS) - than with the unemployment insurance. From Figure 2 we can see there are only $40 \%$ of urban labor forces registered for unemployment insurance while over $60 \%$ of urban labor forces registered with the Basic Pension Scheme. Both BPS and unemployment insurance are compulsory for urban labor forces and in principle the enrollment rate should be similar. Further, figure 3 shows that most of the people who enrolled in Dibao are actually unemployed or flexibly employed, so we can infer that a lot of those who choose to quit unemployment insurance choose to join Dibao instead.

Second, the resource allocation in rural health insurance is also inefficiently too high, which is shown by too high a level of enrollment in rural health insurance and too low utilization of health care services. It is estimated that there are over 100 million people covered by more than one social health insurance program

meetings to coordinate their policies for the recipients of medical assistance program. For mental healthcare, the joint conferences have been held regularly since 2006, where both MCA and MOH sit in the conference.

${ }^{10}$ The direct Dibao benefit, which is in the form of cash transfer, is allocated to urban households whose income is lower than a threshold value. The absolute value of the cash transfer is RMB 4,000 on average annually per recipient in 2012.

${ }^{11}$ The annual benefit level for the unemployment insurance was about RMB 8,800 on average annually per recipient in 2012. Also the enrollees have to pay a premium of the unemployment insurance ( about 1\% of their salary), but they do not need to pay for any premium for Dibao. 


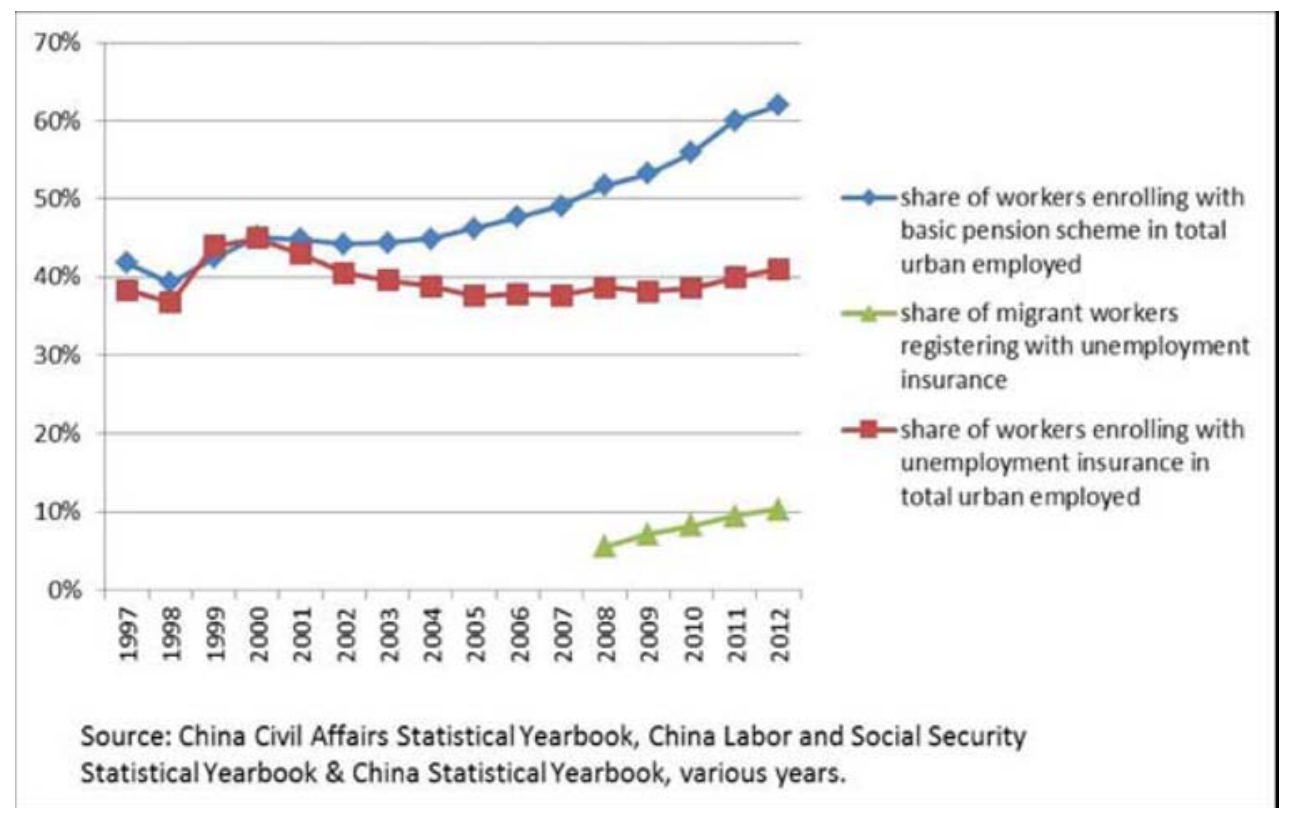

Figure 2: Figure 2. Share of enrollees of unemployment insurance and basic pension scheme in urban labor forces

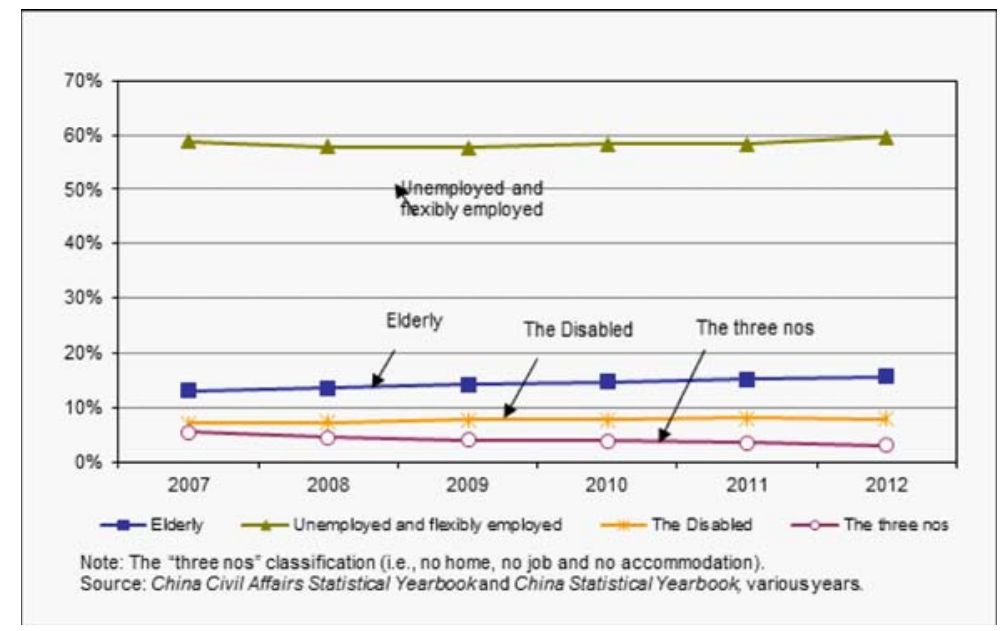

Figure 3: Figure 3. Composition of Beneficiaries under Dibao in urban areas 


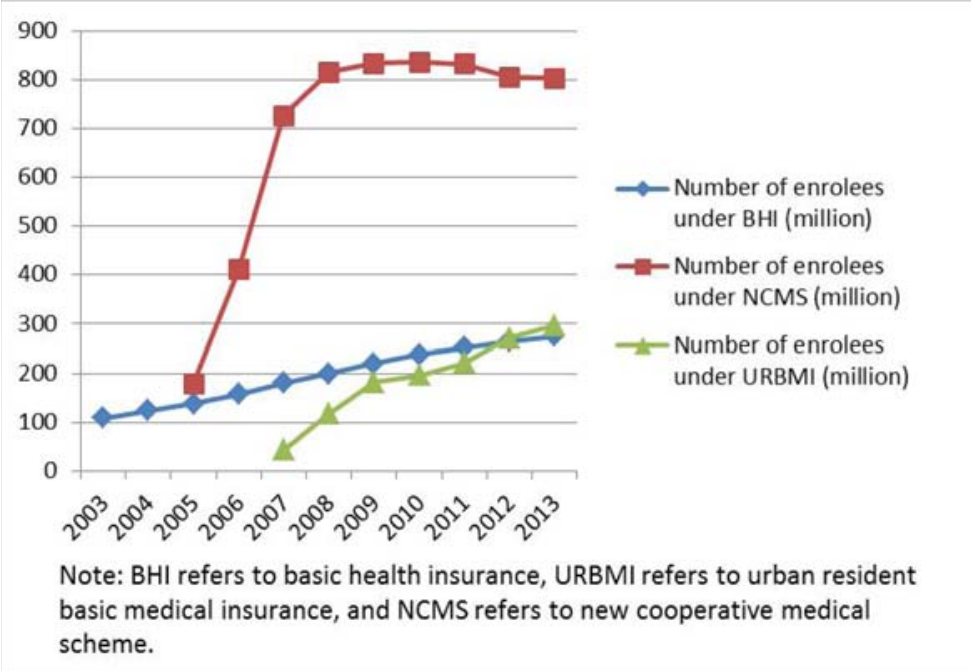

Figure 4: Figure 4. The number of enrollees under different social health insurances

in China ${ }^{12}$. But people can only claim benefit from one of these social health insurance programs if they register with more than one social health insurance. In 2013, the total number of enrollees under three major social health insurance plans is over 1.37 billion which even exceeds the total population in China (1.36 billion, National Bureau of Statistics 2013). However, poor families may still have difficulties paying for healthcare expenditure. The out-of-pocket expenditure is over RMB 1 trillion in 2013 (total health expenditure is about RMB 3 trillion). Figure 4 shows that the coverage of health insurance in China.

Third, the resource allocation in mental health care is inefficiently too low. Figure 5 shows that the government input in mental hospitals as a share of government health and civil affairs budgets is decreasing in both types of hospitals under the MOH and the MCA. The World Health Organization (WHO)'s threshold level is that at least $2 \%$ of total health expenditure should be allocated for mental health care, whereas the share of mental health care expenditure in China is less than this threshold.

\section{Modelling logrolling in autocracies}

The model aims to capture the main features of logrolling in autocracies in the simplest possible way, with a special focus on allocative inefficiency. It is a variant

\footnotetext{
${ }^{12}$ See, http://news.xinhuanet.com/politics/2014-08/15/c_126873413.htm, Accessed Nov 14, 2014
} 


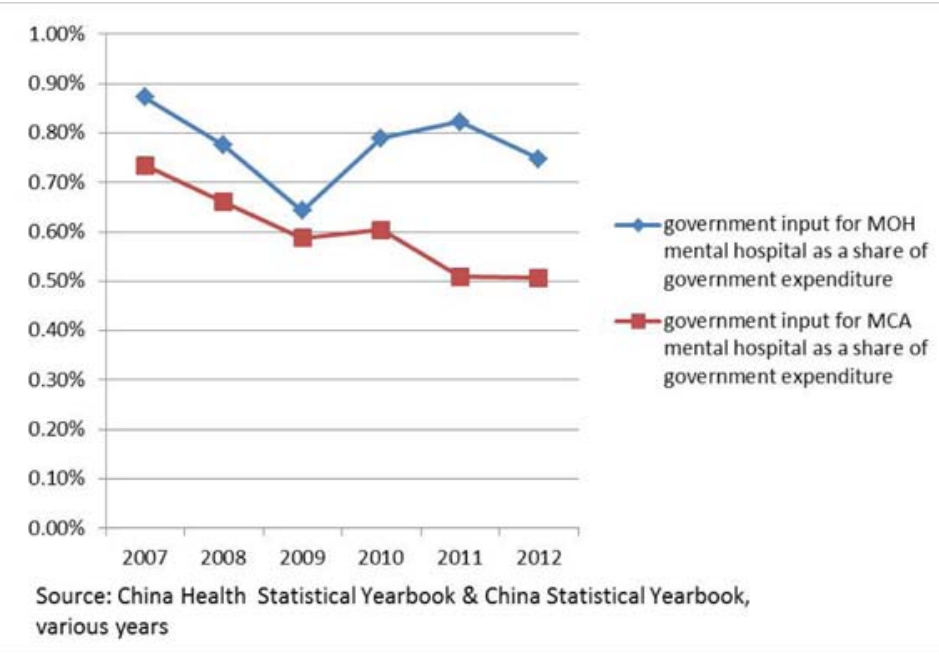

Figure 5: Figure 5. Government inputs in mental hospitals as a share of total government health expenditure

on the legislative bargaining framework initially proposed by Baron and Ferejohn (1989) where each interest group proposes a policy limited to its own sector, and the proposals are constrained by budget balance while the policy vector to be implemented must achieve consensus among all agents. If there is no agreement, a default outcome is implemented by an autocratic leader.

To obtain a simple but effective model, we assume that there is complete information and that the groups' policy proposals are perfectly observable. The situation involves two distinct and homogeneous interest groups $\alpha$ and $\beta$, so that each interest group can be seen as a single player. The interest groups are distinguished by their payoff functions, $U^{i}(h, x, y), \quad i \in\{\alpha, \beta\}$, where $h$ is the public good, $x$ is the private good relevant for group $\alpha$, and $y$ is the private good relevant for group $\beta$. Without loss of generality, we consider $(h, x, y)$ in percentage, so that $(h, x, y) \in[0,1] \times[0,1] \times[0,1]$ and $x+y+h \leq 1$ because of the budget constraint. The payoff functions, $U^{i}(h, x, y)$, are supposed to be smooth and concave such that $\frac{\partial U^{i}(h, x, y)}{\partial h}>0, \frac{\partial U^{\alpha}(h, x, y)}{\partial x}>0, \quad \frac{\partial U^{\alpha}(h, x, y)}{\partial y}=0, \quad \frac{\partial U^{\beta}(h, x, y)}{\partial y}>0$, $\frac{\partial U^{\beta}(h, x, y)}{\partial x}=0$, so that each group is interested in the public good and its own private good but not in the other player's private good. Moreover, $\forall \varepsilon \rightarrow 0^{+}$, $U^{\alpha}(h-\varepsilon, x+\varepsilon, y)>U^{\alpha}(h, x, y)$, and $U^{\beta}(h-\varepsilon, x, y+\varepsilon)>U^{\beta}(h, x, y)$, so that any small redistribution from the public good to the group's private good increases that player's utility. Clearly this is a quite strong assumption, because it is required for any $(x, y) \in[0,1] \times[0,1]$. For our qualitative results it would be enough a weaker local assumption: $\frac{\partial U^{\alpha}(h, x, y)}{\partial x}>\frac{\partial U^{\alpha}(h, x, y)}{\partial h}$ and $\frac{\partial U^{\beta}(h, x, y)}{\partial y}>\frac{\partial U^{\beta}(h, x, y)}{\partial h}$. Both assump- 
tions are ways of modelling the idea that players care more for private than for public good. In the appendix, we will derive closed form solutions for two specific payoff functions, namely quasi-linear and Stone Geary, which satisfy both assumptions. Finally, we assume a form of local symmetry, i.e. $\frac{\partial U^{\alpha}(h, x, y)}{\partial h}=\frac{\partial U^{\beta}(h, x, y)}{\partial h}$, $\frac{\partial U^{\alpha}(h, x, y)}{\partial x}=\frac{\partial U^{\beta}(h, x, y)}{\partial y}$. Using the budget constraint $x+y+h \leq 1$, the payoff functions can be written, with the obvious abuse of notation, as $U^{\alpha}(1-x-y, x)$ or $U^{\beta}(1-y-x, y)$. As a result, our assumptions guarantee that the best reply functions $B R^{\alpha}(y)$ and $B R^{\beta}(x)$ are well defined. The timing of logrolling is as follows:

1. one of the interest groups, say $\alpha^{13}$, is chosen to make its policy proposal $x^{\alpha L} \in[0,1]$

2. the other player, $\beta$, chooses whether to support $\alpha$ 's proposal, i.e. $c^{\beta} \in$ $\{Y, N\}$

3. $\beta$ makes its policy proposal, $y^{\beta L} \in\left[0,1-x^{\alpha L}\right]$;

4. $\alpha$ chooses whether to support or not $\beta$ 's proposal, i.e. it chooses an action $c^{\alpha} \in\{Y, N\}$;

5. if the two players supported each other's proposal, i.e. if $c^{\beta}=c^{\alpha}=Y$, we say a logroll $\left(h^{\alpha L}, x^{\alpha L}, y^{\beta L}\right)$ is forged, where $h^{\alpha L}=1-x^{\alpha L}-y^{\beta L}$, and the proposals in the logroll are implemented;

6. otherwise, if any player rejected the other's policy, we say the logroll failed. Then a default outcome $\left(h^{D}, x^{D}, y^{D}\right)$ is implemented and both players receive a reserve utility, $U^{D}=\theta$, such that $\theta \leq U^{\alpha}(0,1,0)=U^{\beta}(0,0,1)$.

Note that there is only one round of proposals, and amendments are not allowed. ${ }^{14}$ Such a simple frame allows a direct connection between the model's forecasting and econometric analysis. The following picture represents the game tree:

The subgame perfect policy outcomes can be calculated by backward induction. Unfortunately, for the generic payoff functions the conditions are too complex to derive precise equilibrium properties, even if we are able to identify some interesting qualitative properties of the equilibrium outcomes. Thus, we consider two specific payoff functions in order to derive closed form solutions for resource allocation. In this way, we are able to check the implications of autocratic logrolling

\footnotetext{
${ }^{13}$ Using $\beta$ would be no change because of symmetry.

${ }^{14}$ In the jargon of the legislative bargaining literature, we are thus considering a closed rule.
} 


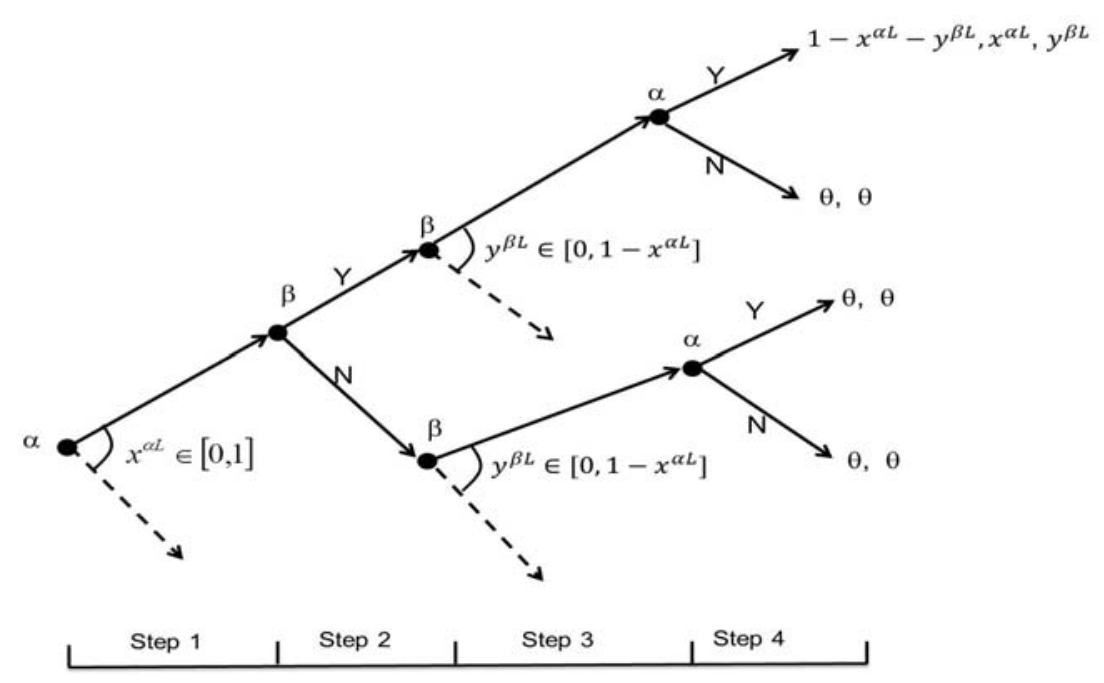

Figure 6: The game of logrolling.

on equilibrium resource allocations. We also derive the general qualitative properties as well as the specific closed form solutions for different decision-making settings. In particular, we study allocations under a utilitarian social planner, by one interest group, and by a simplified version of legislative bargaining.

The two specific payoff functions we consider in order to derive the closed form solutions are:

1. quasi linear payoff functions:

$$
\begin{aligned}
& U^{\alpha}(h, x)=\frac{1}{2} h+\sqrt{x}, \\
& U^{\beta}(h, y)=\frac{1}{2} h+\sqrt{y} .
\end{aligned}
$$

2. Stone Geary payoff functions:

$$
\begin{gathered}
U^{\alpha}(h, x)=\ln (h+1)+2 \ln (x+1) \\
U^{\beta}(h, y)=\ln (h+1)+2 \ln (y+1) .
\end{gathered}
$$

All the calculations and formal details are shown in the Appendix. 


\subsection{Decisions made by autocratic logrolling}

In the general model the conditions on the sequential best replies are too complex to derive precise properties, even if we are able to show that the amount of public good provided through autocratic logrolling can be zero, and in general is suboptimal. The closed form solutions derived for the two examples confirm that the public good allocation under logrolling commonly is null, because the resources allocated to the private goods are inefficiently high.

Solution 1 Suppose the decision is made by autocratic logrolling, then in equilibrium

$$
h^{\alpha A L} \leq h^{E}, \quad x^{\alpha A L} \geq x^{E}, y^{\alpha A L} \lessgtr y^{E}
$$

where $\left(h^{\alpha A L}, x^{\alpha A L}, y^{\alpha A L}\right)$ and $\left(h^{E}, x^{E}, y^{E}\right)$ are respectively the equilibrium allocation under autocratic logrolling and the efficient allocation. In particular

1. if the groups have quasi-linear payoff functions, $\theta \in[0,1]$ and $\alpha$ makes the first proposal, then in equilibrium the allocation of resources is

$$
\left(h^{\alpha A L}, x^{\alpha A L}, y^{\alpha A L}\right)=\left\{\begin{array}{cl}
\left(0,1-\theta^{2}, \theta^{2}\right) & \text { if } \theta \in\left[0, \frac{1}{\sqrt{2}}\right] \\
\left(h^{D}, x^{D}, y^{D}\right) & \text { if } \theta \in\left[\frac{1}{\sqrt{2}}, 1\right] .
\end{array}\right.
$$

2. if the groups have Stone Geary payoff functions, $\theta \in[0, \ln 4]$ and $\alpha$ makes the first proposal, then in equilibrium the allocation of resource is

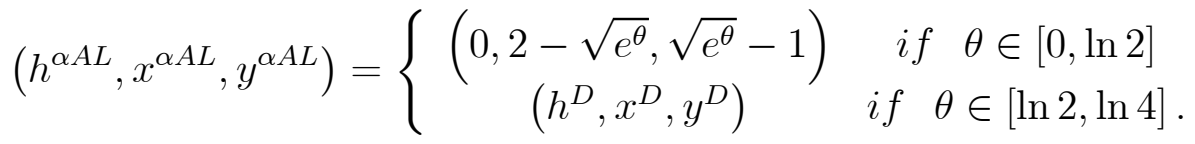

In order to find out the distinctive consequences of autocratic logrolling on policy outcomes, we also derive the policy outcomes under different types of decisionmaking processes as benchmarks, including utilitarianism, dictatorship, and legislative bargaining.

\subsection{The utilitarian efficient benchmark}

Suppose the resource allocation is decided by an utilitarian social planner whose goal is to maximize the sum of groups' payoffs subject to the resource constraint $x+y+h \leq 1$ :

$\max _{(h, x, y)} W(h, x, y)=U^{\alpha}(h, x)+U^{\beta}(h, y) \quad$ s.t. $\quad x+y+h \leq 1 ; x \geq 0 ; y \geq 0 ; h \geq 0$. 
Solution 2 The efficient allocation of public and private goods implemented by an utilitarian social planner satisfies the following inequality

$$
h^{E} \geq x^{E}=y^{E} .
$$

In particular,

1. when the groups quasi-linear payoff functions, then the utilitarian efficient allocation is

$$
\left(h^{E}, x^{E}, y^{E}\right)=\left(\frac{1}{2}, \frac{1}{4}, \frac{1}{4}\right) .
$$

2. when the groups have Stone Geary payoff functions, then the utilitarian efficient allocation is

$$
\left(h^{E}, x^{E}, y^{E}\right)=\left(\frac{1}{3}, \frac{1}{3}, \frac{1}{3}\right) \text {. }
$$

\subsection{An interest group as dictator}

Suppose the policy decision is made by one of the interest groups. Without loss of generality, suppose group $\alpha$ has the decision-making power, then it will simply maximize its group welfare,

$$
\max _{(h, x, y)} U^{\alpha}(h, x) \quad \text { s.t. } h+x+y \leq 1 ; h \geq 0, y \geq 0, x \geq 0 .
$$

As $\alpha$ 's objective function does not depend on $y$, then $y^{\alpha}=0$. However, without further information on the groups' payoff functions it is not possible to say whether the optimal $x^{\alpha}$ is at the boundary, i.e. $x^{\alpha}=1$, or in the interior, i.e. $x^{\alpha} \in(0,1)$. Our examples shows that both cases are possible.

Solution 3 The allocation implemented by $\alpha$ acting as dictator is such that

$$
x^{\alpha}>x^{E} ; \quad h^{\alpha}<h^{E} ; \quad y^{\alpha}<y^{E}
$$

where $\left(h^{E}, x^{E}, y^{E}\right)$ is the efficient allocation. In particular

1. when the groups have quasi-linear payoff functions, the equilibrium allocation implemented by $\alpha$ acting as dictator is

$$
\left(h^{\alpha}, x^{\alpha}, y^{\alpha}\right)=(0,1,0) \text {. }
$$

2. when the groups have Stone Geary payoff functions, the equilibrium allocation implemented by $\alpha$ acting as dictator is

$$
\left(h^{\alpha}, x^{\alpha}, y^{\alpha}\right)=(0,1,0) .
$$

Hence, when a group has full dictator power, there is suboptimal amount of public good because of the excessive spending on its preferred issue. 


\section{Legislative bargaining}

Finally, we consider a simple legislative bargaining process, adapting to this setting the models discussed in the seminal works by Baron and Ferejohn (1989), Persson (1998) and Persson and Tabellini (2000). The timing of this simple legislative bargaining is as follows:

1. one of the interest groups, say $\alpha$, makes a policy proposal $(h, x, y)$;

2. the other group $\beta$ chooses whether to accept or to reject the proposal.

3. If accepted, the proposal is implemented; if not, a default outcome $\left(h^{D}, x^{D}, y^{D}\right)$ is implemented, such that both players will get a reserve utility

$$
U^{D}=\theta<U^{\alpha}(0,1,0)=U^{\beta}(0,0,1) .
$$

$>$ From a formal point of view, this is just an ultimatum game, with the usual standard properties, however the strategic variable is a bundle of public and private goods that makes the strategic interaction slightly more complex since $\alpha$ can use a mix of public and private good to induce $\beta$ to accept the proposal.

Solution 4 The allocation implemented by Legislative Bargaining when $\alpha$ is the first mover is such that

$$
x^{\alpha L B} \geq x^{E} \quad \text { and } \quad y^{\alpha L B} \leq y^{E}
$$

where $\left(h^{E}, x^{E}, y^{E}\right)$ is the efficient allocation. In particular

1. when the groups have quasi-linear payoff functions and $\theta \in[0,1]$, then the equilibrium allocation would depend on the value of reservation utility, $\theta$ :

$$
\left(h^{\alpha L B}, x^{\alpha L B}, y^{\alpha L B}\right)=\left\{\begin{array}{cc}
(2[\sqrt{1-\theta}-(1-\theta)], 1-\theta, 2-\theta-2 \sqrt{1-\theta}) & \text { if } \theta \in\left[0, \frac{3}{4}\right] \\
\left(h^{D}, x^{D}, y^{D}\right) & \text { if } \theta \in\left[\frac{3}{4}, 1\right] .
\end{array}\right.
$$

2. when the groups have Stone Geary payoff functions, then the equilibrium allocation would depend on the value of reservation utility,

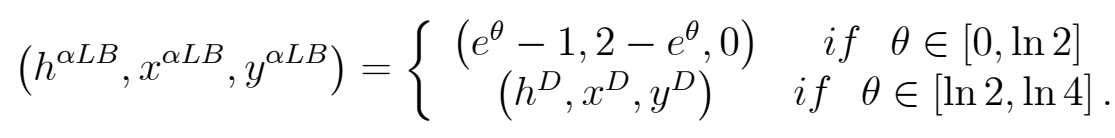

Hence, with Legislative Bargaining there is a first-mover advantage, and in general there is a suboptimal allocation on $h$, because of an excessive spending on its preferred issue by the first mover group. However, note that the distortions are significantly reduced w.r.t. autocratic logrolling. 


\subsection{The distinctive effect of logrolling on policy outcomes}

Table 1 and (7) summarize the equilibrium outcomes under different kinds of decision making rules.

Table 1: Summary of resource allocation under different decision making rules

\begin{tabular}{|c|c|c|}
\hline & \multicolumn{2}{|l|}{ DECISION MAKING RULES } \\
\hline $\begin{array}{l}\text { payoff } \\
\text { functions }\end{array}$ & Autocratic Logrolling & Efficient \\
\hline $\begin{array}{l}\text { quasi- } \\
\text { linear }\end{array}$ & $\left\{\begin{array}{cl}\left(0,1-\theta^{2}, \theta^{2}\right) & \text { if } \theta \in\left[0, \frac{1}{\sqrt{2}}\right] \\
\left(h^{D}, x^{D}, y^{D}\right) & \text { if } \theta \in\left[\frac{1}{\sqrt{2}}, 1\right]\end{array}\right.$ & $\left(\frac{1}{2}, \frac{1}{4}, \frac{1}{4}\right)$ \\
\hline $\begin{array}{l}\text { Stone } \\
\text { Geary }\end{array}$ & $\begin{array}{c}\left(0,2 \sqrt{e^{\theta}}, \sqrt{e^{\theta}}-1\right) \quad \text { if } \quad \theta \in[0, \ln 2] \\
\left(h^{D}, x^{D}, y^{D}\right) \quad \text { if } \theta \in[\ln 2, \ln 4]\end{array}$ & $\left(\frac{1}{3}, \frac{1}{3}, \frac{1}{3}\right)$ \\
\hline & DECISION MAKING RULES & \\
\hline $\begin{array}{l}\text { payoff } \\
\text { functions }\end{array}$ & Legislative Bargaining & $\alpha$ Dictator \\
\hline $\begin{array}{l}\text { quasi- } \\
\text { linear }\end{array}$ & $\begin{array}{cl}(2[\sqrt{1-\theta}-(1-\theta)], 1-\theta, 2-\theta-2 \sqrt{1-\theta}) & \text { if } \theta \in\left[0, \frac{3}{4}\right] \\
\left(h^{D}, x^{D}, y^{D}\right) & \text { if } \theta \in\left[\frac{3}{4}, 1\right]\end{array}$ & $(0,1,0)$ \\
\hline $\begin{array}{l}\text { Stone } \\
\text { Geary }\end{array}$ & $\begin{cases}\left(e^{\theta}-1,2-e^{\theta}, 0\right) & \text { if } \theta \in[0, \ln 2] \\
\left(h^{D}, x^{D}, y^{D}\right) & \text { if } \theta \in[\ln 2, \ln 4]\end{cases}$ & $(0,1,0)$ \\
\hline
\end{tabular}

$$
\begin{aligned}
h^{\alpha} & \leq h^{\alpha A L} \leq h^{\alpha L B} \lessgtr h^{E}, \\
x^{\alpha} & \geq x^{\alpha A L} \geq x^{\alpha L B} \lessgtr x^{E}, \\
y^{\alpha A L} & \lessgtr y^{E} \geq y^{\alpha L B} \geq y^{\alpha} .
\end{aligned}
$$

There are several interesting points worth noting. First, the allocation is generally suboptimal in all situations. But the distortion caused by logrolling is the most serious and systematic, as public good provision is by far the smallest. Under autocratic logrolling, there is excessive spending on both $x$ and $y$, the two issues through which the interest groups trade favors with each other. On the other hand, there exists excessive spending on private issues and consequently an inefficient amount of public good under legislative bargaining, but the inefficiency is not as significant as with autocratic logrolling. Second, the first-mover advantage we observed in the case of legislative bargaining is less important in the case of autocratic logrolling. The first-mover advantage in the existing legislative bargaining literature is treated as the agenda setting power (Persson, 1998). These findings reveal the fundamental difference between autocratic logrolling and legislative bargaining. With legislative bargaining, one player's private gain is at the 
cost of the other player's welfare, hence public good still has a crucial role to induce an agreement. On the other hand, with autocratic logrolling an effective coordination between the interest groups can increase their joint benefit at the cost of society's welfare, and the public good is less relevant to induce agreement. Thus, there is the most severe under-provision of public goods with autocratic logrolling.

In summary, according to our model, autocratic logrolling between $\mathrm{MOH}$ and MCA should result in inefficient policy outcomes — overreaching in both Dibao and rural health insurance programs, and insufficient input in mental health care. The following section will provide empirical tests concerning these issues.

\section{The empirical evidence}

In this section, we provide the rigorous test of logrolling between MCA and $\mathrm{MOH}$. The following subsections will first test the existence of logrolling and then test the consequent inefficiencies.

\subsection{Existence of logrolling among ministries}

In this subsection, we test the existence of logrolling between MCA and $\mathrm{MOH}$ by examing the provincial level budget allocation. Following the literature of logrolling (Stratmann 1992), the following empirical model is used to verify whether there is logrolling between ministries:

$$
\begin{aligned}
& Y_{D, i, t}=\gamma_{D, R} Y_{R, i, t}+\beta_{D} X_{D, i, t-1}+\mu_{i}+\varepsilon_{D, i, t} \\
& Y_{R, i, t}=\gamma_{R, D} Y_{D, i, t}+\beta_{R} X_{R, i, t-1}+\mu_{i}+\varepsilon_{R, i, t}
\end{aligned}
$$

where $Y_{D, i, t}$ is the allocation of resource in MOH's support for Dibao, $Y_{R, i, t}$ is MCA's support for rural health insurance. If logrolling exists between the two ministries, then $\gamma_{D, R}, \gamma_{R, D}$ are expected to be positive and significant, as it indicates that the supports for rural health insurance can explain the supports for Dibao and vice versa. $X_{D, i, t-1}$ and $X_{R, i, t-1}$ are the control variables to capture the factors that can influence resource allocation on Dibao and on rural health insurance. The resource allocation for mental healthcare are also included. Lagged values for these control variables are used since these variables are predetermined. $\mu_{i}$ is the provincial dummy. Note that even if we do not mention the central government in the model, but its role may still significant in influencing the local departments' budgets. We use several empirical methods to take care of the endogeneity issues in the following subsections. 


\subsubsection{Identification strategy}

The biggest endogeneity issue is that some unobserved factors that change over time and affect both inputs from ministries simultaneously (such as the central government's influence, changes of budget policies or new personnel appointment in a ministry) can result in the positive correlation between expenditures on the supportive programs managed by the two ministries. We adopt two identification strategies to find causality. First, we use natural experiment in the form of large scale natural disasters. If the exogenous shocks caused by natural disasters are strong enough, the logrolling process between the two ministries may break down, since the central government will intervene in disaster relief efforts and change the fund allocation pattern for the programs managed by the two ministries. Second, we use a placebo test to see whether the support on one ministry's program from the other ministry is positive related to the placebo policies. If there is no positive correlation between the support on one ministry's program from the other ministry and the placebo policies, our proposition about logrolling are relatively immune to the unobserved factors that change over time.

\subsubsection{Data and estimation methods}

The time span for the data is between 2007 and 2013 (i.e. 7 years). Our data is collected from China Civil Affairs statistical yearbook and China Health statistical yearbook, from various years. A detailed description of variables and descriptive statistics can be found in the appendix. In short, we use the spending to support Dibao as share of the total government health budget to measure MOH's support for Dibao. Similarly, we use the spending to support rural health insurance as a share of the total government civil affairs budget to measure MCA's support for rural health insurance. The spending on mental health care as a share of the total government health and civil affairs expenditure is used to measure the resource allocation on mental health care. The control variables include fiscal expenditure per capita, expenditure for rural health insurance as a share of the budget, the density of hospital doctors, and the urbanization rate. We use the disaster relief expenditure as the placebo for MOH's support for Dibao, and the urban community healthcare expenditure as the placebo for MCA's support for rural health insurance. This is because disaster relief is another social assistance programs managed by MCA and urban community healthcare is another policy area of the $\mathrm{MOH}$, both of which are not likely to be used to exchange favors for the support on rural health insurance and Dibao. Disaster relief expenditure is measured by the share of disaster relief expenditure as a share of the total budget for MCA. Urban community healthcare is measured as the share of beds in urban community healthcare against the total number of hospital beds. 
The regression results in table 2 support the logrolling hypothesis. Column 1 and 2 are the results from the pooled model. MOH's support for Dibao and MCA's support for rural health insurance are positively and significantly associated with each other. A 1\% increase of MCA support for rural health insurance is associated with $2 \%$ increase in MOH's support for Dibao. A $1 \%$ increase of MOH support for Dibao is associated with a $0.04 \%$ increase in MCA's support for rural health insurance. It is likely that these two ministries mutually support the programs managed by their counterpart. The regression table also shows that the fiscal inputs for mental health care are not increased with the MOH and MCA's mutual support for Dibao and rural health insurance.

The incidences of large scale natural disasters are used as exogenous shocks to identify the existence of a logrolling effect. As discussed, when the magnitude of a natural disaster is big enough, logrolling will break down. To test this, we define the magnitude of natural disasters in a year in a province as the ratio of economic losses at a provincial level of local fiscal revenue. The data of the amounts of economic losses are available from the China Civil Affairs Statistical Yearbook. We use $20 \%$ as threshold ratio of economic losses in our data analysis ${ }^{15}$. Column 3 and 4 of table 2 are the regression results for the observations in which there are no large-scale natural disasters. The results show that MOH's support for Dibao and MCA's support for rural health insurance are positively and significantly associated with each other. Column 5 and 6 are the regression results for the observations for which there are large-scale natural disasters. The results show that MOH's support for Dibao and MCA's support for rural health insurance are no longer significantly associated with each other, which implies that with strong external shocks, the logrolling process may breakdown.

Table 3 reports the results when regressing on placebo inputs from these ministries. MOH's support for Dibao and MCA's support for rural health insurance are not positively and significantly associated with each other. The expenditure on disaster relief is negatively associated with the MOH's support for health insurance. Urban community healthcare expenditure is not associated with the MCA's expenditure on rural health insurance. In this case, it is not likely that logrolling is a result of policy shocks from the central government since the expenditure for these supportive programs are not positively associated with disaster relief expenditure and expenditure for urban community health facilities.

\footnotetext{
${ }^{15} 20 \%$ is chosen as threshold value for two reasons. First, the mean of economic losses as the share of fiscal revenue is $20 \%$. Second, the number of observations exceeding this threshold value is not very small.
} 
Table 2: Regression results showing the logrolling between MOH and MCA

\begin{tabular}{|c|c|c|c|c|c|c|}
\hline & (1) & (2) & (3) & (4) & (5) & (6) \\
\hline & $\begin{array}{lr}\text { MOH } & \text { sup- } \\
\text { port } & \text { for } \\
\text { Dibao } & \\
\end{array}$ & $\begin{array}{l}\text { MCA } \\
\text { support } \\
\text { for rural } \\
\text { insurance }\end{array}$ & $\begin{array}{lr}\text { MOH } & \text { sup- } \\
\text { port } & \text { for } \\
\text { Dibao } & \end{array}$ & $\begin{array}{l}\text { MCA sup- } \\
\text { port for RI }\end{array}$ & $\begin{array}{lr}\text { MOH } & \text { sup- } \\
\text { port } & \text { for } \\
\text { Dibao } & \end{array}$ & $\begin{array}{l}\text { MCA sup- } \\
\text { port for RI }\end{array}$ \\
\hline \multirow{2}{*}{$\begin{array}{l}\text { MCA support } \\
\text { for RI }\end{array}$} & $-2.108^{*}$ & & $4.038^{*}$ & & -3.608 & \\
\hline & $(0.889)$ & & $(2.034)$ & & $(2.582)$ & \\
\hline \multirow[t]{2}{*}{$\begin{array}{l}\text { MOH support } \\
\text { for Dibao }\end{array}$} & & -0.0245 & & $0.0537^{* * *}$ & & -0.0185 \\
\hline & & $(0.0167)$ & & $(0.00846)$ & & $(0.0226)$ \\
\hline \multirow{2}{*}{$\begin{array}{l}\mathrm{MOH} \text { input } \\
\text { for mental } \\
\text { care(lagged) }\end{array}$} & 0.362 & & 0.0525 & & $-0.894^{* * *}$ & \\
\hline & $(0.234)$ & & $(0.120)$ & & $(0.265)$ & \\
\hline \multirow{2}{*}{$\begin{array}{l}\text { MCA input } \\
\text { for } \quad \text { mental } \\
\text { care(lagged) }\end{array}$} & & -.0322 & & 0.00584 & & 0.365 \\
\hline & & $(0.0675)$ & & $(0.307)$ & & $(0.186)$ \\
\hline \multirow{2}{*}{$\begin{array}{l}\text { Rural health } \\
\text { insur- } \\
\text { ance(lagged) }\end{array}$} & $-0.0416^{* * *}$ & & $0.0387^{* * *}$ & & $0.0745^{* * *}$ & \\
\hline & $(0.00469)$ & & $(0.00838)$ & & $(0.0119)$ & \\
\hline \multirow{2}{*}{$\begin{array}{l}\text { Doctors } \\
\text { (lagged) }\end{array}$} & $-1.20^{* *}$ & & -0.290 & & -0.509 & \\
\hline & $(0.445)$ & & $(0.582)$ & & $(1.68)$ & \\
\hline \multirow[t]{2}{*}{ Dibao(lagged) } & & $-1.005^{* *}$ & & -0.129 & & $-1.288^{*}$ \\
\hline & & $(0.370)$ & & $(0.137)$ & & $(0.578)$ \\
\hline \multirow{2}{*}{$\begin{array}{l}\text { Urbanization } \\
\text { (lagged) }\end{array}$} & & $4.063^{* * *}$ & & $-1.342^{* * *}$ & & $-2.283^{*}$ \\
\hline & & $(0.983)$ & & $(0.241)$ & & $(0.905)$ \\
\hline \multirow{2}{*}{$\begin{array}{l}\text { Fiscal expendi- } \\
\text { ture }\end{array}$} & $0.102^{*}$ & -0.00289 & 0.115 & -0.0137 & $0.696^{*}$ & $0.0970^{* *}$ \\
\hline & $(0.0430)$ & $(0.0132)$ & $(0.0826)$ & $(0.00711)$ & $(0.273)$ & $(0.0348)$ \\
\hline $\begin{array}{l}\text { Provincial } \\
\text { fixed effect }\end{array}$ & Yes & Yes & Yes & Yes & No & No \\
\hline$N$ & 155 & 155 & 128 & 128 & 27 & 27 \\
\hline$R^{2}$ & 0.9447 & 0.7903 & 0.6083 & 0.5256 & 0.7521 & 0.4321 \\
\hline
\end{tabular}

Standard errors in parentheses

$* \mathrm{p}<0.05,{ }^{* *} \mathrm{p}<0.01,{ }^{* * *} \mathrm{p}<0.001$ 
Table 3: A placebo regression results showing the logrolling between $\mathrm{MOH}$ and MCA

\begin{tabular}{|c|c|c|}
\hline & $\begin{array}{c}\text { (1) } \\
\text { MOH support for Dibao }\end{array}$ & $\begin{array}{l}(2) \\
\text { MCA support for rural } \\
\text { insurance }\end{array}$ \\
\hline Disaster relief expenditure & $\begin{array}{c}-2.108^{*} \\
(0.889)\end{array}$ & \\
\hline Urban community healthcare & & $\begin{array}{l}-0.0245 \\
(0.0167)\end{array}$ \\
\hline $\mathrm{MOH}$ input for mental care(lagged) & $\begin{array}{c}0.362 \\
(0.234)\end{array}$ & \\
\hline MCA input for mental care (lagged) & & $\begin{array}{l}-.0322 \\
(0.0675)\end{array}$ \\
\hline Rural health insurance (lagged) & $\begin{array}{c}-0.0416^{* * *} \\
(0.00469)\end{array}$ & \\
\hline Doctors(lagged) & $\begin{array}{c}-1.20^{* *} \\
(0.445)\end{array}$ & \\
\hline Dibao(lagged) & & $\begin{array}{l}-1.005^{* *} \\
(0.370)\end{array}$ \\
\hline Urbanization (lagged) & & $\begin{array}{l}4.063^{* * *} \\
(0.983)\end{array}$ \\
\hline Fiscal expenditure & $\begin{array}{c}0.102^{*} \\
(0.0430)\end{array}$ & $\begin{array}{c}-0.00289 \\
(0.0132)\end{array}$ \\
\hline Provincial fixed effect & Yes & Yes \\
\hline$N$ & 155 & 155 \\
\hline$R^{2}$ & 0.9466 & 0.7798 \\
\hline
\end{tabular}

Standard errors in parentheses

${ }^{*} \mathrm{p}<0.05,{ }^{* *} \mathrm{p}<0.01,{ }^{* * *} \mathrm{p}<0.001$ 


\subsection{Inefficiency in the high benefit associated with "Dibao"}

In this subsection, we show that, as a result of logrolling among the ministries, the benefit associated with urban medical assistance even crowds out unemployment insurance.

\subsubsection{Data and estimation methods}

In order to test whether the benefit from urban medical assistance program would crowd out unemployment insurance, we estimate the following model:

$$
U I_{i, t}=\beta D_{i b a o_{i, t}}+\gamma M e d_{-} A s s_{i, t}+\delta X_{i, t}+\omega_{t}+e_{i, t} .
$$

where the dependent variable $U I_{i, t}$ is the ratio between the number of enrollees of unemployment insurance and the number of employees in city $i$ during year $t$. $D_{i b a o_{i, t}}$ is the amount of cash benefit per recipient in the city $i$ during year $t$, which measures the direct benefit of Dibao. Our aim of including this variable is to test whether the increasing direct benefit of Dibao crowds out unemployment insurance. Med_Assi,t is the amount of government health expenditure per person in the city $i$ during year $t$, which is the proxy to measure the complementary benefit of Dibao from urban medical assistance programs. Medical assistance expenditure has been counted as a sub-category of government health expenditure since 2007. $X_{i, t}$ are the covariates including average income, fiscal expenditure per capita, unemployment rate and gross product per capita of the city and $\omega_{t}$ yearly dummy variables. If there is a crowding-out effect between urban medical assistance programs and UI, the number of enrollees in UI should decrease with the benefit level of urban medical assistance.

Two data sources are used in this section. The first data source is China City Statistical Yearbook (NBS: various years). This dataset includes observations of 282 cities (prefecture level) in 26 out of 31 provinces (i.e. 4 provincial level city and Tibet are not included). The data for enrollees for unemployment insurance is only available for 2011, 2012 and 2013. The second dataset is data reported by MCA about Dibao at the prefecture level, which was collected from the website of MCA. Both of our dataset include data for 282 out of 332 prefecture cities in total in China. The city statistical yearbook only reports data in 282 cities. Excluding the observations with missing data, we end up with 789 observations covering three years. A prefecture city usually has both urban (i.e. city district) and rural areas (i.e. county), and since we are interested in urban social programs, data in this study are at city district level in these prefecture cities. Since the city level UI enrollment data is only available for the last three years, we pool all the observations together. A detailed description of variables and descriptive statistics can be found in the appendix. 
Omitted variable bias and simultaneity need to be taken into consideration in the estimation. We use two ways to cross-check our results. First, we use government education expenditure level as a placebo to check whether the urban education assistance, which is managed by the ministry of education and also targets poor urban households, can have a similar effect as urban medical assistance. The ministry of education, unlike the ministry of health, has little overlapping business with the ministry of civil affairs. Our model, in this case, will predict that the level of education assistance, which is measured by the proxy variable government education expenditure, will not crowd out the enrollees in unemployment insurance. Second, we use the enrollment rate of BPS as a reference point to measure the crowding out effect. BPS, the same as unemployment insurance, is compulsory for employees in urban formal sectors. Urban employees working in the informal sector, similar to the case of unemployment insurance, can opt out of the BPS. However, unlike unemployment insurance in which the benefit level is flat and not earning related, as a pension program, the benefit level of BPS is earning-related. In other words, the more people contribute to BPS, the benefit level of BPS will be higher. For urban informal workers with a reasonable income, the benefit from BPS may be much larger than the benefit from Dibao and urban medical assistance. In this case, an increasing benefit level from urban medial assistance is less likely to crowd out enrollees in BPS. The enrollment rate of BPS can also serve as a reference point to check the scale of the crowding-out effect between urban medical assistance and unemployment insurance.

Another caveat in interpreting the result is that we use the proxy variable of government health expenditure to measure the benefit level of urban medical assistance. The main reason for using the proxy is that there is no public data available for the city level urban medical assistance data. Besides this, government expenditure for urban medical assistance is an important component of government health expenditure in China, since the main goal of government health expenditure is to provide basic healthcare for all households ${ }^{16}$. Therefore, the benefit level of urban medical assistance must be positively correlated with government health expenditure. However, when an imprecise measure of a variable is used in a regression model, the model inevitably contains a measurement error. Under the classical errors-in-variables (CEV) assumption, the estimated effect will be attenuated. This kind of attenuation bias is not likely to cause serious problems to the result, because if a significant effect of government health expenditure is observed, it means the real effect of urban medical assistance is of an even greater magnitude. However, if the CEV assumption is violated, for example, in the case

\footnotetext{
${ }^{16}$ For example, a recent report about the government plan for health reform between 2011 and 2015 explicitly states that the role of government is to provide basic health care service. http://finance.china.com.cn/industry/medicine/20120726/904196.shtml, accessed Jan 28, 2015.
} 
where the measurement error is negatively correlated with government health expenditure and the weight is larger than for government health expenditure, it is possible to observe a negative effect of government health expenditure even when the true effect of urban medial assistance is positive. Due to the data limitations, it is not possible to test the validity of the CEV assumption.

\subsubsection{Results}

We find that the higher direct benefit of Dibao is not associated with less enrollment in unemployment insurance, but the higher benefits of urban medical assistance is associated with less enrollment in unemployment insurance. This implies that many urban residents have avoided unemployment insurance because the aggregate benefit from Dibao (but not the direct benefit from Dibao) is higher than the benefit from unemployment insurance. Our finding also suggests that the crowding-out effect is a result of logrolling, since the aggregate benefit from Dibao has been pulled up by the complementary social assistance programs from other ministries such as urban medical assistance (i.e. MOH-supported social assistance). The regression results are shown in the Table 4 .

Column (1) shows the results where there is a regression in the number of enrollees in UI on the benefit level of Dibao. The coefficient for direct Dibao benefit is not statistically significant, which shows that the direct benefit level of Dibao is not correlated with the number of enrollees covered by UI. The coefficient for the level of GDP is significant and positive. The coefficient for the size of the informal sector is negative and significant. These results are consistent with our expectation that a more developed and formal economy will have more people enrolled in UI.

In column (2), MOH supported social assistance (i.e. medical assistance) is included as an independent variable. The coefficient for Dibao benefit remains statistically insignificant. It confirms the earlier result that Dibao is not crowding out enrollees in UI. The coefficient of MOH-supported social assistance is negative and significant. One standard deviation above the mean of $\mathrm{MOH}$-supported social assistance means that the number of enrollees under UI will be decreased by 3.3 percent of the labor force. This result suggests that the urban medical social assistance may actually crowd out the enrollees in UI. Unemployment rate, gross product and fiscal expenditure are positively associated with enrollment in UI, and the size of the informal sector is negatively associated with the enrollment rate of enrollment rate in UI.

To check the robustness of our result, in column (3), we add government education expenditure as a regressor measuring the level of education assistance. The coefficient for direct Dibao benefit is still not significant, which suggests there is no crowding-out effect from Dibao. Education expenditure, as a proxy measurement 
Table 4: Unemployment Insurance and Medical assisitance Regression result

\begin{tabular}{lcccc}
\hline \hline & $(1)$ & $(2)$ & $(3)$ & $(4)$ \\
& ui & ui & ui & bps/ui \\
\hline direct benefit of Dibao & -1.987 & -1.683 & -2.079 & -0.0250 \\
& $(1.392)$ & $(1.385)$ & $(1.400)$ & $(0.0773)$ \\
MOH-supported social assistance & & $-20.65^{* *}$ & & $1.027^{*}$ \\
education assistance & & $(6.804)$ & & $(0.445)$ \\
fiscal expenditure & & & 1.935 & -0.286 \\
& 0.431 & $0.543^{*}$ & 0.424 & -0.0184 \\
average income & $(0.250)$ & $(0.250)$ & $(0.250)$ & $(0.0138)$ \\
& & & & \\
informal sector size & $0.428^{* * *}$ & $0.491^{* * *}$ & $0.413^{* *}$ & -0.00777 \\
& $(0.124)$ & $(0.125)$ & $(0.126)$ & $(0.00707)$ \\
gross product & 0.309 & -1.335 & 0.476 & 0.0542 \\
& $(5.802)$ & $(5.752)$ & $(5.809)$ & $(0.309)$ \\
unemployment & $0.0872^{*}$ & $0.0912^{*}$ & $0.0809^{*}$ & -0.00016 \\
& $(0.0369)$ & $(0.0363)$ & $(0.0381)$ & $(0.00188)$ \\
\hline Year Dummy & & & & \\
\hline$N$ & 31.38 & 28.32 & 32.01 & $6.273^{* *}$ \\
overall. $R^{2}$ & $(38.49)$ & $(38.50)$ & $(38.51)$ & $(2.309)$ \\
\hline \hline
\end{tabular}

Standard errors in parentheses

${ }^{*} \mathrm{p}<.05,{ }^{* *} \mathrm{p}<0.01,{ }^{* * *} \mathrm{p}<0.001$ 
Table 5: Unemployment Insurance and Medical assisitance Regression result when there were no large scale natrual disasters

\begin{tabular}{lcccc}
\hline \hline & $(1)$ & $(2)$ & $(3)$ & $(4)$ \\
& ui & ui & ui & bps/ui \\
\hline direct benefit of Dibao & -2.643 & -2.230 & -2.778 & 0.0182 \\
& $(1.487)$ & $(1.478)$ & $(1.498)$ & $(0.0795)$ \\
MOH-supported social assistance & & $-21.92^{* *}$ & & $1.028^{*}$ \\
education assistance & & $(7.005)$ & & $(0.444)$ \\
fiscal expenditure & & & 2.201 & -0.273 \\
& & & $(2.961)$ & $(0.184)$ \\
average income & 0.356 & 0.475 & 0.348 & -0.0214 \\
& $(0.262)$ & $(0.262)$ & $(0.262)$ & $(0.0141)$ \\
informal sector size & $0.492^{* * *}$ & $0.562^{* * *}$ & $0.475^{* * *}$ & -0.00983 \\
& $(0.131)$ & $(0.132)$ & $(0.133)$ & $(0.00720)$ \\
gross product & -1.412 & -3.306 & -1.158 & 0.142 \\
& $(6.191)$ & $(6.125)$ & $(6.202)$ & $(0.315)$ \\
unemployment & $0.0961^{*}$ & $0.0990^{* *}$ & $0.0892^{*}$ & -0.00021 \\
Year Dummy & $(0.0382)$ & $(0.0375)$ & $(0.0394)$ & $(0.00191)$ \\
\hline$N$ & & & & \\
$R^{2}$ & 25.44 & 22.85 & 26.18 & $6.184^{* *}$ \\
\hline \hline
\end{tabular}

Standard errors in parentheses

${ }^{*} \mathrm{p}<.05,{ }^{* *} \mathrm{p}<0.01,{ }^{* * *} \mathrm{p}<0.001$ 
Table 6: Unemployment Insurance and Medical assisitance Regression result when there were large scale natural disasters

\begin{tabular}{lcccc}
\hline \hline & $(1)$ & $(2)$ & $(3)$ & $(4)$ \\
& ui & ui & ui & bps/ui \\
\hline direct benefit of Dibao & $13.97^{* *}$ & $14.37^{* *}$ & $14.68^{* *}$ & $-0.697^{*}$ \\
& $(4.577)$ & $(4.780)$ & $(4.654)$ & $(0.278)$ \\
MOH-supported social assistance & & 2.342 & & -0.945 \\
education assistance & & $(34.20)$ & & $(2.185)$ \\
fiscal expenditure & & & 5.041 & -0.525 \\
& & & $(10.23)$ & $(0.546)$ \\
average income & 1.564 & 1.512 & 1.342 & -0.118 \\
& $(1.073)$ & $(1.083)$ & $(1.111)$ & $(0.0804)$ \\
informal sector size & 0.0470 & 0.0701 & 0.0915 & 0.0129 \\
& $(0.401)$ & $(0.411)$ & $(0.414)$ & $(0.0200)$ \\
gross product & & & & \\
& -14.02 & -14.34 & -14.93 & 0.815 \\
unemployment & $(14.75)$ & $(15.14)$ & $(15.28)$ & $(0.738)$ \\
& & & & \\
\hline Year Dummy & -0.0575 & -0.0623 & -0.0716 & 0.00066 \\
\hline$N$ & $(0.0920)$ & $(0.0940)$ & $(0.0941)$ & $(0.00674)$ \\
$R^{2}$ & 188.7 & 185.9 & 188.1 & 6.047 \\
\hline \hline
\end{tabular}

Standard errors in parentheses

${ }^{*} \mathrm{p}<.05,{ }^{* *} \mathrm{p}<0.01,{ }^{* * *} \mathrm{p}<0.001$ 
for the benefit of education assistance, is not significant in column (3). To cross check the validity of our results, we change the dependent variable as the ratio of the enrollees in the BPS and UI. The result is shown in column (4). As in previous models, the direct benefit of Dibao is not statistically significant. The result in column (4) also shows that the level of MOH-supported social assistance is positively and significantly correlated with the ratio between the enrollment number in the BPS and UI, which implies that a higher benefit level of MOH-supported social assistance is associated with a higher ratio of the number of enrollees covered by BPS and UI. In other words, the gap of enrollee number between BPS and UI is increasing with the level of MOH-supported social assistance. This result implies that many workers in the informal sector enrolled with BPS may opt out of UI.

Table 5 and 6 use exogenous shocks to identify the causal relation between the logrolling process and inefficiency in Dibao. As in the earlier discussion, if the economic losses of a natural disaster as a share of fiscal revenue in a province exceeds 20\%, we consider it an exogenous shock for the logrolling process. For a province with large-scale natural disasters, central governments may intervene and the logrolling process is likely to break down. Table 5 reports the regressing results based on observations for which the scale of the natural disaster is not very big (729 observations). Similar to the results reported earlier, in table 5, Dibao itself is not a significant determinant for crowded out enrollees in UI. Also, MOHsupported social assistance is negatively associated with the number of enrollees in UI. Table 6 reports the results for those cities where local provinces suffered a large scale natural disaster (60 observations). Interestingly, Dibao's direct benefit level now moves in the same direction as the number of enrollees in UI. Also, MOHsupported social assistance is no longer a significant determinant for the number of enrollees in UI. It implies that there was no crowding-out effect when there were large-scale natural disasters. These results provide robust support for the causal link between the logrolling process and inefficiency in implementing Dibao and UI programs.

\subsection{Inefficiency in enrollment for rural health insurance}

Since only logrolling produces distortion towards excessive expenditure on both the private issues of the two interest groups, we argue that, as a result of logrolling among the ministries, there must be an overreaching in the policy area of rural health insurance. Rural health insurance is recognized as the priority policy area for $\mathrm{MOH}$. Under logrolling, MCA supports $\mathrm{MOH}$ by subsidizing enrollees in rural health insurance using its own medical assistance fund. In this subsection, we will test the inefficiency (overreaching) in rural health insurance. 


\subsubsection{Data and estimation methods}

Our hypothesis is that rural health insurance is managed in an inefficient way when the MCA subsidizes some rural residents to enroll in the rural health insurance. With an increasing number of enrollees in the rural health insurance, if utilization of healthcare services are not increased, it indicates some inefficiency since access to healthcare is not improved with better financial coverage. We also use the number of those enrollees covered by urban health insurance, whose premiums are financed by urban medical assistance programs, as a control group. Since health insurance in urban areas is managed by the ministry of human resources and social security, if there is an exchange of interests between MCA and $\mathrm{MOH}$ in the context of rural health insurance, urban medical assistance will have a different impact on health service utilization compared to rural medical assistance. In order to test the above hypothesis. We estimate the following model:

$$
\text { Utilization }_{i, t}=\beta \text { Asstnce }_{i, t}+\delta X_{i, t}+\mu_{i}+\omega_{t}+e_{i, t} .
$$

We use provincial level data to explore the relation between the medical assistance fund and the rural health insurance plan. The data is collected from the China Health statistical yearbook as well as the China Civil Affairs statistical yearbook, over various years. The time span of our data is between 2009 and 2013 . We study this time period for two reasons. First, the recent round of health reforms started in 2009. Second, the urban medical assistance data has only been available since 2009 . Utilization $_{i, t}$ denotes outcomes for health service utilization including inpatient and outpatient service volume as well as the revenue of service providers in province $i$ in year $t$. Asstnce $_{i, t}$ refers to the number of recipients of medical assistance which covers their insurance premium in rural and urban areas in province $i$ in year $t$. Control variables $X_{i, t}$ include the supply side determinants: the number of beds in the hospitals and the number of doctors. Demand side determinants are also included as regressors: coverage of major health insurances, average income level and the proportion of the population which is 65 years or older in province $i$ in year t. $\omega_{t}$ and $\mu_{i}$ corresponds to year and provincial dummy variables. All variables are weighted by local population. Supply side determinants are important for health service utilization since the number of doctors and hospital beds are major inputs for health care service production. Health insurance and average level of income determine the demand for health services. People with insurance coverage or with a higher income are more likely to utilize health care service compared to people without health insurance or lower incomes. Also, the share of the population which is 65 years or older is also important for healthcare service demand since people over 65 years old utilize health services much more compared to other groups of the population. 


\subsubsection{Results}

The following table 7 show that the number of people subsidized for paying premiums for rural health insurance is not positively associated with the revenue, volume of inpatient services in hospitals as well as outpatient services. Column (1) shows that the number of those enrollees from poor households under rural health insurance, whose insurance premium is covered by medical assistance fund, is not associated with the utilization of inpatient services. Column (2) shows that the number of enrollees covered by medical assistance funds is not associated with the revenue the service provides. From column (3) and (4), it shows that the number of enrollees financed by rural medical assistance is also not significantly associated with the volume of outpatient services provided by hospitals and primary care clinics. However, these regression results suggest that the effectiveness of rural medical assistance is in question. The rural health insurance plan is managed by the Minister of Health and the number of rural health insurance enrollees financed by rural medical assistance programs is not associated with the utilization of services as well as revenue generated by providing services. Therefore, our hypothesis is supported and there is inefficiency in the policy area of rural health insurance.

\subsection{Inefficiency in the supply of infrastructure in mental health care}

$>$ From the logrolling model, we predict that there will be insufficient government input in mental health care infrastructure, which can be seen as the public good in the model. While mental healthcare services are provided by hospitals under $\mathrm{MOH}$ and MCA, mental health care is not the priority issue for both MCA and $\mathrm{MOH}$ to invest in. In this subsection, we will test the inefficiency (insufficient input) in mental health care infrastructure. If we can show that under-supply of infrastructure rather than the demand side reasons (e.g. income, education, insurance status) is the major constraint for mental health care, then the existence of such inefficiency is proven.

The supply of infrastructure includes both physical inputs, such as the number of beds in the hospitals, and nonphysical inputs, such as the number of medical professionals working in the hospitals. The beds occupancy rates were $96.5 \%$ and $80 \%$ in $\mathrm{MOH}$ and MCA mental hospitals in 2013.

\subsubsection{Data and estimation methods}

Our hypothesis is that the level of mental healthcare infrastructure is positively associated with the utilization rate of mental healthcare services. We use two 
Table 7: Volume of inpatient services and outpatients service hospital revenue

\begin{tabular}{|c|c|c|c|c|}
\hline & $\begin{array}{c}(1) \\
\text { inpatients }\end{array}$ & $\begin{array}{c}(2) \\
\text { revenue }\end{array}$ & $\begin{array}{c}(3) \\
\text { hospital outpatient }\end{array}$ & $\begin{array}{c}(4) \\
\text { clinic outpatient }\end{array}$ \\
\hline rural insurance (assisted) & $\begin{array}{l}0.0269 \\
(0.293)\end{array}$ & $\begin{array}{c}0.472 \\
(0.894)\end{array}$ & $\begin{array}{c}6.334 \\
(25.60)\end{array}$ & $\begin{array}{c}38.08 \\
(32.64)\end{array}$ \\
\hline urban insurance (assisted) & $\begin{array}{c}1.124 \\
(0.612)\end{array}$ & $\begin{array}{c}1.043 \\
(1.866)\end{array}$ & $\begin{array}{l}-55.87 \\
(53.74)\end{array}$ & $\begin{array}{l}-42.77 \\
(68.53)\end{array}$ \\
\hline doctors & $\begin{array}{c}0.138 \\
(1.334)\end{array}$ & $\begin{array}{c}17.40^{* * *} \\
(4.066)\end{array}$ & $\begin{array}{c}127.0 \\
(116.1)\end{array}$ & $\begin{array}{l}83.19 \\
(148.1)\end{array}$ \\
\hline beds & $\begin{array}{c}19.38^{* * *} \\
(1.469)\end{array}$ & $\begin{array}{l}-0.386 \\
(4.477)\end{array}$ & & \\
\hline age65 & $\begin{array}{l}-28.87 \\
(56.32)\end{array}$ & $\begin{array}{l}-53.58 \\
(171.7)\end{array}$ & $\begin{array}{c}3762.6 \\
(4749.9)\end{array}$ & $\begin{array}{l}-2406.2 \\
(6057.1)\end{array}$ \\
\hline bhi & $\begin{array}{c}0.329 \\
(0.262)\end{array}$ & $\begin{array}{c}3.423^{* * *} \\
(0.800)\end{array}$ & $\begin{array}{c}134.2^{* * * *} \\
(23.03)\end{array}$ & $\begin{array}{c}-77.27^{* *} \\
(29.37)\end{array}$ \\
\hline $\mathrm{cms}$ & $\begin{array}{c}0.141 \\
(0.0750)\end{array}$ & $\begin{array}{c}0.639^{* *} \\
(0.229)\end{array}$ & $\begin{array}{l}1.942 \\
(6.532)\end{array}$ & $\begin{array}{l}-0.363 \\
(8.330)\end{array}$ \\
\hline urbmi & $\begin{array}{c}0.0213 \\
(0.0315)\end{array}$ & $\begin{array}{c}0.0874 \\
(0.0959)\end{array}$ & $\begin{array}{l}-3.179 \\
(2.751)\end{array}$ & $\begin{array}{c}2.249 \\
(3.509)\end{array}$ \\
\hline urban disposable income & $\begin{array}{l}1.622^{*} \\
(0.697)\end{array}$ & $\begin{array}{c}3.307 \\
(2.123)\end{array}$ & $\begin{array}{l}-51.80 \\
(60.26)\end{array}$ & $\begin{array}{c}118.6 \\
(76.85)\end{array}$ \\
\hline rural disposable income & $\begin{array}{l}-1.627 \\
(1.207)\end{array}$ & $\begin{array}{c}5.844 \\
(3.679)\end{array}$ & $\begin{array}{l}-111.4 \\
(101.4)\end{array}$ & $\begin{array}{c}126.6 \\
(129.2)\end{array}$ \\
\hline Provincial Dummy & Yes & Yes & Yes & Yes \\
\hline Year Dummy & Yes & Yes & Yes & Yes \\
\hline $\begin{array}{c}N \\
\text { adj. } R^{2}\end{array}$ & $\begin{array}{c}149 \\
0.966\end{array}$ & $\begin{array}{c}149 \\
0.890\end{array}$ & $\begin{array}{c}149 \\
0.638\end{array}$ & $\begin{array}{c}149 \\
0.704\end{array}$ \\
\hline
\end{tabular}

Standard errors in parentheses

$* \mathrm{p}<.05,{ }^{* *} \mathrm{p}<0.01, * * * \mathrm{p}<0.001$ 
groups of hospitals to test this hypothesis. One group consists of those mental hospitals managed by $\mathrm{MOH}$ and the other group consists of mental hospitals managed by MCA. This is a provincial level data analysis. The time span for the data is between 2007 and 2013 (i.e. 7 years). We collect the data from the China Health Statistical Yearbook and China Civil Affairs Statistical Yearbook, for various years. If there is under-investment on the infrastructure, it will show that the marginal effect of infrastructure is positive and the magnitude of this effect will be large. The limitation of the data is that we do not know the number of psychiatrists at the provincial level. However, we can use the number of doctors in $\mathrm{MOH}$ hospitals and the number of medical staffs in MCA mental hospitals as a proxy measurement for the number of psychiatrists in $\mathrm{MOH}$ and $\mathrm{MCA}$ mental hospitals.

$$
\text { Mentalcare_utilization }_{i, t}=\beta \text { Infrastructure }_{i, t}+\delta X_{i, t}+\mu_{i}+\omega_{t}+e_{i, t}
$$

Mentalcare_utilization ${ }_{i, t}$ denotes outcomes for the utilization of mental healthcare services (inpatient) in MOH and MCA hospitals in province $i$ in year $t$. Infrastructure $_{i, t}$ denotes the number of beds as well as the number of medical staffs in the MOH and MCA hospitals in province $i$ in year $t . \omega_{t}$ and $\mu_{i}$ are year and provincial dummy variables. The control variables include education level, insurance coverage, local average income level and the share of industry in the local economy. All of these variables are demand-side determinants for mental healthcare services. All variables are weighted by population size. As in the previous section, a higher level of income and better insurance, which are included as regressors, may imply a higher demand for mental healthcare. The control variable "education ratio" denotes the share of tertiary education in population, which in the literature implies a higher demand for mental healthcare since educated people are more informed. The share of industry in the local economy, denoted by "industry ratio", is also positively associated with the utilization of mental healthcare. according to the literature (Chen, et al 2014).

\subsubsection{Results}

Table 8 shows the regression results.

Column (1) and (2) are the benchmark results for the level of infrastructure and utilization of health services. In column (1), the coefficients for the number of $\mathrm{MOH}$ hospital beds is positive and statistically significant at the $1 \%$ level. According to column (1), one more bed per million people will increase utilization of mental healthcare inpatient services by about 4.7 per million people in $\mathrm{MOH}$ hospitals. Column (2) shows that the number of beds in MCA hospitals is not 
Table 8: Regression results showing the determinants of utlization of mental healthcare

\begin{tabular}{|c|c|c|c|c|}
\hline & $\begin{array}{c}\text { (1) } \\
\mathrm{MOH} \text { inpatients }\end{array}$ & $\begin{array}{c}(2) \\
\text { MCA inpatients }\end{array}$ & $\begin{array}{c}(3) \\
\mathrm{MOH} \text { inpatients }\end{array}$ & $\begin{array}{c}\text { (4) } \\
\text { MCA inpatients }\end{array}$ \\
\hline doctors & $\begin{array}{l}279.38^{*} \\
(116.21)\end{array}$ & $\begin{array}{l}4.474^{*} \\
(2.124)\end{array}$ & $\begin{array}{l}87.216 \\
(88.64)\end{array}$ & $\begin{array}{c}5.279^{* *} \\
(1.789)\end{array}$ \\
\hline beds & $\begin{array}{c}4.737^{* * *} \\
(1.137)\end{array}$ & $\begin{array}{c}1.655 \\
(1.083)\end{array}$ & $\begin{array}{c}5.097^{* * *} \\
(0.847)\end{array}$ & $\begin{array}{c}1.280 \\
(1.043)\end{array}$ \\
\hline education ratio & & & $\begin{array}{l}-5.262 \\
(3.287)\end{array}$ & $\begin{array}{c}1.931 \\
(1.433)\end{array}$ \\
\hline urban insurance & & & $\begin{array}{c}0.601 \\
(1.215)\end{array}$ & $\begin{array}{c}0.779 \\
(0.537)\end{array}$ \\
\hline industry ratio & & & $\begin{array}{c}3.227 \\
(2.199)\end{array}$ & $\begin{array}{l}-0.571 \\
(1.819)\end{array}$ \\
\hline disposable income & & & $\begin{array}{l}15.29 \\
(9.531)\end{array}$ & $\begin{array}{c}0.542 \\
(4.653)\end{array}$ \\
\hline Year Dummy & Yes & Yes & Yes & Yes \\
\hline Provincial Dummy & Yes & Yes & Yes & Yes \\
\hline$N$ & 212 & 200 & 212 & 200 \\
\hline $\operatorname{adj.} R^{2}$ & 0.794 & 0.129 & 0.811 & 0.122 \\
\hline
\end{tabular}

Standard errors in parentheses

${ }^{*} \mathrm{p}<.05,{ }^{* *} \mathrm{p}<0.01,{ }^{* * *} \mathrm{p}<0.001$ 
statistically significant. However, the number of medical doctors is another significant determinant. In column (1) and (2), the coefficients for the number of medical staff in both $\mathrm{MCA}$ and $\mathrm{MOH}$ is positive and statistically significant at the $5 \%$ level. One more medical staff per thousand people will increase the utilization of mental healthcare inpatient services by about 279 and 4,595 per million people in $\mathrm{MOH}$ and MCA hospitals. Demand-side determinants are added in column (3) and (4). It turns out that in column (3) and (4), none of these demand-side determinants such as income, urbanization, the size of manufacture sector, education level is statistically significant. The number of beds and the number of medical staffs remain significant determinants for $\mathrm{MOH}$ and MCA mental hospitals. $\mathrm{MOH}$ hospitals are more likely to be constrained by the number of beds and MCA hospitals are more likely to be constrained by the number of staffs. It implies that in $\mathrm{MOH}$ and MCA mental hospitals, with under-supplied physical and nonphysical infrastructures, demand-side reasons are not significant. Therefore, our hypothesis is supported. The regression results in this table, therefore, show that the supplyside constraint is a major reason for the under-treatment of patients with mental diseases in both MCA and MOH hospitals.

\section{Conclusion}

This paper focused on logrolling in autocracies among vertically fragmented interest groups, to pick apart the black-box of decision making in dictatorships. It showed that some generalized patterns of decision-making in autocracies can be revealed by a close scrutiny of the embedding complex institutional environment and of the actual operation of policy-making. In particular, it focused on China where bureaucratic interest groups are separate vertical organizations reaching down from Beijing to the provinces and cities that operate with little discipline or coordination from above. Because of this, the decision making in contemporary China exemplifies a process of autocratic logrolling. The paper studied the effects of the logrolling of parochial interest groups on state policies and social welfare in China, both theoretically and empirically. The theoretical model, although very simple, helped to focus on a specific distortion in resource allocation because of logrolling. The theoretical predictions helped to identify the regressions to test the effects of logrolling in autocracies, while the econometric results confirmed the theoretic predictions in a very robust way. In particular, the paper provides empirical evidence by studying the logrolling between the Ministry of Civil Affairs and the Ministry of Health, showing that there is inefficiency due to excessive spending on "Dibao" and on rural health insurance, and insufficient input in infrastructure building in mental health care. These results confirm that due to logrolling, private policies tend to be inefficiently high and policies excluded from logrolling tend to 
be inefficiently low.

\section{References}

[1] Alesina, A. and Rosenthal, H. (1996). A Theory of Divided Government, Econometrica, 64: 1311-1341.

[2] Austen-Smith, D., and Banks, J. (1988). Elections, Coalitions, and Legislative Outcomes. The American Political Science Review, 82: 405-422.

[3] Baron D. and Ferejohn J. (1989). Bargaining in Legislatures. American Political Science Review, 83: 1181-1206.

[4] Buchanan J. and Tullock G. (1962). The Calculus of Consent: Logical Foundations of Constitutional Democracy. University of Michigan Press.

[5] China Association of Social Workers (2010). Reports on Development of Social Work in China. Beijing: Social Sciences Academic Press.

[6] Copelovitch M. (2010). Master or Servant? Common Agency and the Political Economy of IMF Lending. International Studies Quarterly, 54(1): 49-77.

[7] Crombez C. (2000). Spatial models of logrolling in the European Union. European Journal of Political Economy, 16: 707-737.

[8] Enelow, James (1986). The Stability of Logrolling: An Expectations Approach. Public Choice, 51(3): 285-294.

[9] Evans, Diana (1994). Policy and Pork: The Use of Pork Barrel Projects to Build Policy Coalitions in the House of Representatives. American Journal of Political Science, 38 (4): 894-917.

[10] Huang, Yanzhong (2013). Governing Health in Contemporary China, Abingdon and New York: Routledge.

[11] Irwin, Douglas and Kroszner, Randall (1996). Log-rolling and Economic Interests in the Passage of the Smoot-Hawley Tariff. Carnegie-Rochester Conference Series on Public Policy, 45: 173-200.

[12] Ma, N, et, al (2014) Allocation of mental health facilities and psychiatric beds in China in 2010, China Mental Health Journal, 26(12): 885-9.

[13] Miller, Nicholas (1977). Logrolling, vote trading, and the paradox of voting: A game-theoretical overview. Public Choice, 30(1): 51-75 
[14] Ministry of Civil Affairs (2011). Civil Affairs Statistical Yearbook. Beijing.

[15] National Bureau of Statistics(NBS) (Various Years). China City Statistical Yearbook: various years. Beijing: China Statistical Press.

[16] Persson T. and Tabellini G. (2000). Political Economics: Explaining Economic Policy. London: MIT Press.

[17] Persson, T. (1998), Economic Policy and Special Interest Politics. The Economic Journal, 108: 310-327.

[18] Riker W. and Brahms S. (1973). The Paradox of Vote Trading. American Political Science Review, 67: 1235-1247.

[19] Snyder J. (1991). Myths of Empire: Domestic Politics and International Ambition. New York: Cornell University Press.

[20] Stratmann T. (1992). The Effects of Logrolling on Congressional Voting. The American Economic Review, 82(5): 1162-1176

[21] Stratmann T. (1995). Logrolling in the U.S. Congress. Economic Inquiry, 33(3): 441-456.

[22] Shirk S. (1993). The Political Logic of Economic Reform in China. California: University of California Press.

[23] Shirk S. (2014). The Domestic Context of Chinese Foreign Security Policies. In Pekkanen S., Ravenhill C., and Foot R. eds. The Oxford Handbook of the International Relations of Asia. New York: Oxford University Press. pp. 391-410. DOI: 10.1093/oxfordhb/9780199916245.013.0020

[24] Weingast B. and Marshall W. (1988). The Industrial Organization of Congress; or, Why Legislatures, Like Firms, Are Not Organized as Markets. Journal of Political Economy, 96(1): 132-163. 


\section{Part I}

\section{Appendix}

\section{The Subgame Perfect Equilibria of the Logrolling Model}

In this section, we calculate the subgame perfect equilibria of our logrolling model, and we compare these equilibrium allocations with other relevant benchmarks derived by different interaction models. Moreover, we calculate the precise equilibrium allocations when the players have specific quasi linear and Stone Geary payoff functions:

\section{1. quasi linear payoff functions:}

$$
\begin{aligned}
& U^{\alpha}(h, x)=\frac{1}{2} h+\sqrt{x}, \\
& U^{\beta}(h, y)=\frac{1}{2} h+\sqrt{y} .
\end{aligned}
$$

\section{Stone Geary payoff functions:}

$$
\begin{gathered}
U^{\alpha}(h, x)=\ln (h+1)+2 \ln (x+1) \\
U^{\beta}(h, y)=\ln (h+1)+2 \ln (y+1) .
\end{gathered}
$$

The reserve utility when no logroll is forged, is assumed to be smaller than the utility when a player get all the available resources. For the quasi linear payoffs we get

$$
U^{D}=\theta<U^{\alpha}(0,1,0)=U^{\beta}(0,0,1)=1 \Leftrightarrow \theta \in[0,1],
$$

and for the Stone Geary functions

$$
U^{D}=\theta<U^{\alpha}(0,1,0)=U^{\beta}(0,0,1)=2 \ln 2 \Leftrightarrow \theta \in[0, \ln 4] .
$$

Since our payoff functions are symmetric by assumption, from now on we will consider just $\alpha$ as the player moving first.

It is easy to show that the quasi linear payoff functions satisfies our assumption that small redistributions from the public good to the group's private good increases that player's utility:

$$
U^{\alpha}(h-\varepsilon, x+\varepsilon, y) \geq U^{\alpha}(h, x, y) \Leftrightarrow \frac{1}{2}(h-\varepsilon)+\sqrt{x+\varepsilon} \geq \frac{1}{2} h+\sqrt{x} \Leftrightarrow
$$




$$
\Leftrightarrow \sqrt{x+\varepsilon} \geq \frac{1}{2} \varepsilon+\sqrt{x} \Leftrightarrow x \leq\left(1-\frac{1}{4} \varepsilon\right)^{2}
$$

which is always satisfied for any $\varepsilon \rightarrow 0^{+}$. Similarly, also our Stone Geary payoff functions would satisfy this condition:

$$
\begin{aligned}
& U^{\alpha}(h-\varepsilon, x+\varepsilon, y) \geq U^{\alpha}(h, x, y) \Leftrightarrow \ln (h+1-\varepsilon)+2 \ln (x+1+\varepsilon) \geq \ln (h+1)+2 \ln (x+1) \Leftrightarrow \\
& \Leftrightarrow(h+1-\varepsilon)(x+1+\varepsilon)^{2} \geq(h+1)(x+1)^{2} \Leftrightarrow \\
& \Leftrightarrow(x-\varepsilon)^{2}-2 h x-2 h-1+\varepsilon(1-h) \leq 0 \Leftrightarrow \\
& \Leftrightarrow h+\varepsilon-\sqrt{2 h-\varepsilon+3 h \varepsilon+h^{2}+1} \leq x \leq h+\varepsilon-\sqrt{2 h-\varepsilon+3 h \varepsilon+h^{2}+1}
\end{aligned}
$$

which is always satisfied for any $\varepsilon \rightarrow 0^{+}$.

Clearly this is a quite strong assumption, since it is global in $(h, x)$. For our qualitative results it would be enough a weaker local assumption, i.e. $\forall(h, x) \in$ $[0,1] \times[0,1] \quad \frac{\partial U^{\alpha}(h, x, y)}{\partial x} \geq \frac{\partial U^{\alpha}(h, x, y)}{\partial h}$, however the previous global conditions make the results more crisp. Of course, both the payoff functions of our examples satisfy this local condition:

$$
\frac{\partial}{\partial x}\left(\frac{1}{2} h+\sqrt{x}\right)=\frac{1}{2 \sqrt{x}} \geq \frac{\partial}{\partial h}\left(\frac{1}{2} h+\sqrt{x}\right)=\frac{1}{2} \Leftrightarrow x \leq 1
$$

which is always satisfied for any $(h, x) \in[0,1] \times[0,1]$;

$$
\begin{gathered}
\frac{\partial}{\partial x}(\log (1+h)+2 \log (x+1))=\frac{2}{x+1} \geq \frac{\partial}{\partial h}(\log (1+h)+2 \log (x+1))=\frac{1}{1+h} \Leftrightarrow \\
\Leftrightarrow x+1 \leq 2+2 h \Leftrightarrow 2 h-x \geq-1
\end{gathered}
$$

which is always satisfied for any $(h, x) \in[0,1] \times[0,1]$.

\subsection{Autocratic Logrolling}

The equilibrium policy outcome is calculated by backward induction.

Step $4 \alpha$ chooses between to accept the policy proposed by $\beta$ and the default policy. Let $H^{3}$ represents the history of the players' choices in step 1 to step 3 and let us distinguish two different sets of third stage subgames $H^{3}$, whether in step $2 \beta$ rejected or accepted $\alpha$ 's proposal. If $N \in H^{3}$, then $\alpha$ 's choice is irrelevant as both players will get the default outcome. Hence $\alpha$ sequential best reply at $H^{3}$ is

$$
\forall H^{3} \text { s.t. } N \in H^{3} \quad S B R^{\alpha}\left(H^{3}\right) \in\{Y, N\} \text {. }
$$


On the other hand, if $Y \in H^{3}$, then

$$
S B R^{\alpha}\left(H^{3}\right)=Y \Leftrightarrow\left\{\begin{array}{cc}
U^{\alpha}\left(1-x-y, y ; Y \mid H^{3}\right) \geq U^{D}=\theta & \text { Incentive constraint } \\
x+y \leq 1 & \text { Resource constraint } \\
x \in[0,1] & \text { Boundary constraint } \\
y \in[0,1] & \text { Boundary constraint }
\end{array} \Leftrightarrow y \in G^{\alpha}\right.
$$

where by construction $y \in G^{\alpha}(x, \theta) \subseteq[0,1]$ is the compact interval that solves the inequalities of IL and it is called $\alpha$ 's "incentive logrolling constraint". Then, we can write the following sequential best reply for $\alpha$ :

$$
S B R^{\alpha}\left(H^{3}\right) \in\left\{\begin{array}{cll}
\{Y, N\} & \text { if } & H^{3} \in\{(x, N, y):(x, y) \in[0,1] \times[0,1]\} \\
\{Y\} & \text { if } H^{3} \in\left\{(x, Y, y): y \in G^{\alpha}(x, \theta)\right\} \\
\{N\} & \text { if } H^{3} \in\left\{(x, Y, y): y \notin G^{\alpha}(x, \theta)\right\}
\end{array}\right.
$$

Two properties of the incentive logrolling correspondence

$$
G^{\alpha}:[0,1] \times\left[0, \Theta^{\max }\right] \rightrightarrows[0,1]
$$

are crucial:

1. non monotonicity in $x$

$$
x^{\prime} \geq x \nRightarrow G^{\alpha}\left(x^{\prime}, \theta\right) \subseteq G^{\alpha}(x, \theta)
$$

2. monotonicity in $\theta$

$$
\theta^{\prime}>\theta \Rightarrow G^{\alpha}\left(x, \theta^{\prime}\right) \subset G^{\alpha}(x, \theta)
$$

as our examples will show.

Suppose that $U^{\alpha}$ is simple enough so that we have what we might call the "functional case" $(\mathrm{FC})^{17}$, i.e.

$$
y \in G^{\alpha}(x, \theta) \Leftrightarrow 0 \leq y \leq F^{\alpha}(x, \theta),
$$

then $F$ as a function of $x$ can be non monotone, while as a function of $\theta$ is surely decreasing, i.e.

$$
\frac{\partial F^{\alpha}(x, \theta)}{\partial x} \lessgtr 0 \text { and } \frac{\partial F^{\alpha}(x, \theta)}{\partial \theta}<0 .
$$

These properties are important because they imply that $x$ can not be trivially used as strategic variable to relax the incentive logrolling constraint, while an increase in the reservation payoff for sure makes more difficult to forge a logrolling deal.

\footnotetext{
${ }^{17}$ In principle $G^{\alpha}(x, \theta)$ may be disconneted, which may complicate the definition the upper boundary.
} 
Step 3 In any second stage subgame $H^{2}, \beta$ chooses $y$ to maximize his utility subject to $S B R^{\alpha}\left(H^{3}\right)$. Again, we distinguish two different set of subgames, i.e. whether in step $2 \beta$ rejected or accepted $\alpha$ 's proposal. If $N \in H^{2}$, then $\beta$ 's choice is irrelevant as both players will anyway get the default outcome. Hence

$$
\forall H^{2} \quad \text { s.t. } N \in H^{2} \quad S B R^{\beta}\left(H^{2}\right) \in[0,1]
$$

otherwise

$$
\forall H^{2} \text { s.t. } Y \in H^{2} \quad S B R^{\beta}\left(H^{2}\right) \in \operatorname{argmax}_{y} U^{\beta}\left(h, y ; S B R^{\alpha}\left(H^{3}\right) \mid H^{2}\right) .
$$

To solve this maximization problem, note that $\beta$ can always guarantee itself the reservation payoff $\theta$ by refusing any proposal. Otherwise, we have a standard maximization problem in $y$ depending on $\theta$ and on the $x$ that characterizes subgame $H^{2} \in[0,1] \times\{Y, N\}$ :

$$
\begin{aligned}
& \max _{y} U^{\beta}(1-x-y, y) \\
\text { s.t. } & y \in G^{\alpha}(x, \theta)
\end{aligned}
$$

Let consider the unconstrained maximization problem: by assumption the FOCs are necessary and sufficient, so that

$$
\frac{d U^{\beta}(1-x-y, y)}{d y}=-\frac{\partial U^{\beta}}{\partial h}+\frac{\partial U^{\beta}}{\partial y} \geq 0 \Leftrightarrow \frac{\partial U^{\beta}}{\partial y} \geq \frac{\partial U^{\beta}}{\partial h}
$$

which by assumption is always satisfied. Hence we have a corner solution:

$$
y^{\max }(x ; \theta)=\max G^{\alpha}(x, \theta)
$$

Note that when FC holds, then

$$
\max G^{\alpha}(x, \theta)=F^{\alpha}(x, \theta) .
$$

From the properties of IL constraint we can conclude that

$$
\frac{\partial y^{\max }(x ; \theta)}{\partial \theta} \leq 0
$$

while unfortunately

$$
\frac{\partial y^{\max }(x ; \theta)}{\partial x} \lessgtr 0 .
$$

Hence the local sequential best response of $\beta$ is

$$
\widetilde{S B R}^{\beta}\left(H^{2}\right) \in\left\{\begin{array}{cl}
\left\{\max G^{\alpha}(x, \theta)\right\} & \text { if } H^{2} \in\{(x, Y): x \in[0,1]\} \\
{[0,1]} & \text { if } H^{2} \in\{(x, N): x \in[0,1]\}
\end{array}\right.
$$


Now, we should check whether the local sequential best response it is also global, i.e. that:

$$
U^{\beta}\left(1-x-\widetilde{S B R}^{\beta}\left(H^{2}\right), \widetilde{S B R}^{\beta}\left(H^{2}\right) ; S B R^{\alpha}\left(H^{3}\right) \mid H^{2}\right) \geq \theta
$$

otherwise

$$
S B R^{\beta}\left(H^{2}\right)=\left\{y \in[0,1] \mid y>\max G^{\alpha}(x, \theta)\right\} .
$$

From the previous properties, we can conclude that

$$
\forall x \in[0,1], \exists \bar{\theta} \quad \text { s.t. } \forall \theta \geq \bar{\theta} S B R^{\beta}\left(H^{2}\right)=\left\{y \in[0,1] \mid y>\max G^{\alpha}(x, \theta)\right\}
$$

however the equivalent property doesn't hold wrt $x$, i.e. there exists no threshold for $\widehat{x}$ such that $\forall x \geq \widehat{x}$ it is better for $\beta$ to reject the logrolling proposal, i.e. the intervals of $x$ such that $\beta$ rejects the logrolling proposal might be disjoint because of the non monotonicity of $G^{\alpha}(x, \theta)$ in $x$. Hence, the only way to write the general global sequential best reply is

$$
S B R^{\beta}\left(H^{2}\right) \in\left\{\begin{array}{cc}
\left\{\max G^{\alpha}(x, \theta)\right\} & \text { if } H^{2} \text { s.t. } \widetilde{U}^{\beta}\left(H^{2}\right) \geq \theta \\
\left\{y \in[0,1] \mid y>\max G^{\alpha}(x, \theta)\right\} & \text { if } H^{2} \text { s.t. } \widetilde{U}^{\beta}\left(H^{2}\right)<\theta \\
{[0,1]} & \text { if } H^{2} \in\{(x, N): x \in[0,1]\}
\end{array}\right.
$$

where

$$
\widetilde{U}^{\beta}\left(H^{2}\right):=U^{\beta}\left(1-x-\widetilde{S B R}^{\beta}\left(H^{2}\right), \widetilde{S B R}^{\beta}\left(H^{2}\right) ; S B R^{\alpha}\left(H^{3}\right) \mid H^{2}\right) .
$$

Step $2 \beta$ chooses whether to support $\alpha$ proposal on $x$, for any $x \in[0,1]$, anticipating $S B R^{\beta}\left(H^{2}\right)$ and $S B R^{\alpha}\left(H^{3}\right)$. In particular, suppose

$$
S B R^{\alpha}(\underbrace{x, S B R^{\beta}(x), S B R^{\beta}(\underbrace{x, S B R^{\beta}(x)}_{H^{2}})}_{H^{3}})=Y,
$$

then $S_{B R} R^{\beta}(x)=Y$ if and only if

$$
\begin{gathered}
U^{\beta}\left(1-x-S B R^{\beta}\left(H^{2}\right), S B R^{\beta}\left(H^{2}\right) ; Y, S B R^{\alpha}\left(H^{3}\right)=Y \mid x\right) \geq \\
\geq U^{\beta}\left(1-x-S B R^{\beta}\left(H^{2}\right), S B R^{\beta}\left(H^{2}\right) ; N, S B R^{\alpha}\left(H^{3}\right)=Y \mid x\right)=U^{D}=\theta \Leftrightarrow x \in G^{\beta}\left(\theta ; G^{\alpha}(x, \theta\right.
\end{gathered}
$$


where by construction $x \in G^{\beta}\left(\theta ; G^{\alpha}(x, \theta)\right)$ is the solution of the inequality ICL-1, that - restricted to $[0,1]$ - is called $\alpha$ 's "incentive logrolling constraint". Then, we can write the following sequential best reply for $\beta$ :

$$
S B R^{\beta}(x) \in \begin{cases}\{Y\} & \text { if } \quad x \in G^{\beta}\left(\theta ; G^{\alpha}(x, \theta)\right) \cap[0,1] \\ \{N\} & \text { if } x \notin G^{\beta}\left(\theta ; G^{\alpha}(x, \theta)\right) \cap[0,1] .\end{cases}
$$

Clearly, the analysis of the behavior of the set

$$
G^{\beta}\left(\theta ; G^{\alpha}(x, \theta)\right) \cap[0,1]
$$

is difficult: it may be disconnected and even empty, the only property that again holds for sure is decreasing monotonicity in $\theta$.

Step $1 \alpha$ chooses $x \in[0,1]$ to maximize

$$
U^{\alpha}\left(1-x-S B R^{\beta}\left(H^{2}\right), x ; S B R^{\beta}(x), S B R^{\alpha}\left(H^{3}\right)\right)
$$

that we will denote by $U^{\alpha}(x \mid \theta)$ to simplify. Again, suppose

$$
S B R^{\alpha}(\underbrace{x, S B R^{\beta}(x), S B R^{\beta}(\underbrace{x, S B R^{\beta}(x)}_{H^{2}}}_{H^{3}})=Y,
$$

then

$$
U^{\alpha}(x \mid \theta)=\left\{\begin{array}{cl}
U^{\alpha}(1-x-\underbrace{S B R^{\beta}\left(H^{2}\right)}_{y \text { funct. of } x}, x ; Y, S B R^{\alpha}\left(H^{3}\right)=Y \mid x) & \text { if } x \in G^{\beta}\left(\theta ; G^{\alpha}(x, \theta)\right) \\
\theta & \text { if } x \notin G^{\beta}\left(\theta ; G^{\alpha}(x, \theta)\right) .
\end{array}\right.
$$

The interesting case is when

$$
x \in G^{\beta}\left(\theta ; G^{\alpha}(x, \theta)\right)
$$

then the FOCs are

$$
\frac{\partial U^{\alpha}}{\partial h} \frac{\partial(1-x-\underbrace{S B R^{\beta}\left(H^{2}\right)}_{y \text { funct. of } x})}{\partial x}+\frac{\partial U^{\alpha}}{\partial x} \geq 0 .
$$

To derive, if possible, some properties from these FOCs, suppose $S B R^{\beta}\left(H^{2}\right) \in$ $\left\{\max G^{\alpha}(x, \theta)\right\}$, otherwise the objective function is constant. Thus there are two possibilities: 
i. if

$$
\max G^{\alpha}(x, \theta)=1-x
$$

then

$$
\frac{\partial U^{\alpha}}{\partial h} \frac{\partial(1-x-\underbrace{S B R^{\beta}\left(H^{2}\right)}_{1-x})}{\partial x}+\frac{\partial U^{\alpha}}{\partial x}=\frac{\partial U^{\alpha}}{\partial x} \geq 0 \quad \forall x \in G^{\beta}\left(\theta ; G^{\alpha}(x, \theta)\right) ;
$$

hence the local maximum is

$$
x^{\max }=\max G^{\beta}\left(\theta ; G^{\alpha}(x, \theta)\right) ;
$$

Note that in this case, the players decide either not to forge a logroll agreement or to provide no public good $h$ using all the available resources for private goods $x$ and $y$;

ii. if

$$
\max G^{\alpha}(x, \theta) \neq 1-x
$$

then

$$
\frac{\partial U^{\alpha}}{\partial h} \frac{\partial(1-x-\underbrace{S B R^{\beta}\left(H^{2}\right)}_{G^{\alpha}(x, \theta) \neq 1-x})}{\partial x}+\frac{\partial U^{\alpha}}{\partial x} \geq 0
$$

might have an interior or a boundary solution. However, under regularity conditions on the payoff functions, $\max \left\{G^{\alpha}(x, \theta) \neq 1-x\right\}=F^{\alpha}(x, \theta)$ so that

$$
\underbrace{\frac{\partial U^{\alpha}}{\partial h}}_{>0}[\underbrace{-1}_{<0}+\underbrace{\frac{\partial F^{\alpha}(x, \theta)}{\partial x}}_{\gtrless 0}]+\underbrace{\frac{\partial U^{\alpha}}{\partial x}}_{>0} \lessgtr 0 .
$$

Therefore, we can't conclude that even in this case the amount of public good provided through logrolling will be zero, however it will be clearly suboptimal because

$$
[\underbrace{-1}_{<0}+\underbrace{\frac{\partial F^{\alpha}(x, \theta)}{\partial x}}_{\gtrless 0}]<1 .
$$

Unfortunately, as explained before, the behavior of $F^{\alpha}(x, \theta)$ is too complex to derive further precise equilibrium properties from the above conditions. For this reason in the following, we consider two examples with specific payoff functions in order to derive closed form solutions and thus the exact equilibrium resource allocation as a guide for the empirical investigation. 


\subsubsection{Example 1: Quasi Linear Payoff Functions}

The equilibrium policy outcome is calculated by backward induction.

Step $4 \alpha$ chooses between to accept the policy proposed by $\beta$ and the default policy. Let distinguish two different sets of third stage subgames $H^{3}$, whether in step $2 \beta$ rejected or accepted $\alpha$ 's proposal. If $N \in H^{3}$, then $\alpha$ 's choice is irrelevant as both players will get the default outcome. Hence $\alpha$ sequential best reply at $H^{3}$ is

$$
\forall H^{3} \text { s.t. } N \in H^{3} \quad S B R^{\alpha}\left(H^{3}\right) \in\{Y, N\} .
$$

On the other hand, if $Y \in H^{3}$, then

$$
S B R^{\alpha}\left(H^{3}\right)=Y \Leftrightarrow U^{\alpha}\left(1-x-y, y ; Y \mid H^{3}\right) \geq U^{D} \Leftrightarrow \frac{1}{2} h+\sqrt{x} \geq \theta \Leftrightarrow
$$

and, using the resource constraint $h=1-x-y$, which is binding since $U^{\alpha}$ is increasing in $(h, x)$.

$$
\Leftrightarrow \frac{1}{2}(1-x-y)+\sqrt{x} \geq \theta \Leftrightarrow \widetilde{y} \leq \min \{1-x+2 \sqrt{x}-2 \theta, 1-x\} .
$$

First, note that, using the variables' boundary constraints

$$
1-x+2 \sqrt{x}-2 \theta \leq 1-x \Leftrightarrow x \in\left[0, \theta^{2}\right]
$$

and that

$$
\begin{gathered}
1-x+2 \sqrt{x}-2 \theta \geq 0 \Leftrightarrow x-2 \sqrt{x}-(1-2 \theta) \leq 0 \Leftrightarrow \\
\Leftrightarrow\left\{\begin{array}{cl}
x \in[0,1] & \theta \in\left[0, \frac{1}{2}\right] \\
x \in[3-2 \theta-2 \sqrt{2(1-\theta)}, 1] & \theta \in\left[\frac{1}{2}, 1\right]
\end{array}\right.
\end{gathered}
$$

finally

$$
\theta \in\left[\frac{1}{2}, 1\right] \Rightarrow 3-2 \theta-2 \sqrt{2(1-\theta)}<\theta^{2}
$$

Hence, we can write $\alpha$ 's "incentive logrolling constraint" (12) for our quasi linear payoffs as follows:

$$
S B R^{\alpha}\left(H^{3}\right)=Y
$$

if and only if

1. when $\theta \in\left[0, \frac{1}{2}\right]$ then

$$
\tilde{y} \in\left\{\begin{array}{cl}
{[0,1-x+2 \sqrt{x}-2 \theta]} & \text { if } x \in\left[0, \theta^{2}\right] \\
{[0,1-x]} & \text { if } x \in\left[\theta^{2}, 1\right]
\end{array}\right.
$$


2. when $\theta \in\left[\frac{1}{2}, 1\right]$ then

$$
\widetilde{y} \in\left\{\begin{array}{ccc}
\{0\} & \text { if } & x \in[0,3-2 \theta-2 \sqrt{2(1-\theta)} \\
{[0,1-x+2 \sqrt{x}-2 \theta]} & \text { if } & x \in\left[3-2 \theta-2 \sqrt{2(1-\theta)}, \theta^{2}\right. \\
{[0,1-x]} & & \text { if } x \in\left[\theta^{2}, 1\right]
\end{array}\right.
$$

Hence, we get the following sequential best reply for $\alpha$ :

1. if $\theta \in\left[0, \frac{1}{2}\right]$

$$
S B R^{\alpha}\left(H^{3}\right) \in\left\{\begin{array}{cr}
\{Y, N\} & \text { if } H^{3} \in\{(x, N, y):(x, y) \in[0,1] \times[0,1-x]\} \\
\{Y\} & \text { if } H^{3} \in\left\{(x, Y, y):(x, y) \in\left[0, \theta^{2}\right] \times[0,1-x+2 \sqrt{x}-2 \theta]\right\} \\
\{N\} & \text { if } H^{3} \in\left\{(x, Y, y):(x, y) \in\left[0, \theta^{2}\right] \times[1-x+2 \sqrt{x}-2 \theta, 1-x]\right\} \\
\{Y\} & \text { if } H^{3} \in\left\{(x, Y, y):(x, y) \in\left[\theta^{2}, 1\right] \times[0,1-x]\right\} \\
\{N\} & \text { if } H^{3} \in\left\{(x, Y, y):(x, y) \in\left[\theta^{2}, 1\right] \times[1-x, 1]\right\}
\end{array}\right.
$$

2. if $\theta \in\left[\frac{1}{2}, 1\right]$

$$
\in\left\{\begin{array}{cc}
\{Y, N\} & H^{3} \in\{(x, N, y):(x, y) \in[0,1] \times[0,1-x]\} \\
\{Y\} & H^{3} \in\{(x, Y, y):(x, y) \in[0,3-2 \theta-2 \sqrt{2(1-\theta)}] \times\{0\}\} \\
\{N\} & H^{3} \in\{(x, Y, y):(x, y) \in[0,3-2 \theta-2 \sqrt{2(1-\theta)}] \times(0,1]\} \\
\{Y\} & H^{3} \in\left\{(x, Y, y):(x, y) \in\left[3-2 \theta-2 \sqrt{2(1-\theta)}, \theta^{2}\right] \times[0,1-x+2 \sqrt{x}-2 \theta]\right\} \\
\{N\} & H^{3} \in\left\{(x, Y, y):(x, y) \in\left[3-2 \theta-2 \sqrt{2(1-\theta)}, \theta^{2}\right] \times[1-x+2 \sqrt{x}-2 \theta, 1-x\right. \\
\{Y\} & H^{3} \in\left\{(x, Y, y):(x, y) \in\left[\theta^{2}, 1\right] \times[0,1-x]\right\} \\
\{N\} & H^{3} \in\left\{(x, Y, y):(x, y) \in\left[\theta^{2}, 1\right] \times[1-x, 1]\right\}
\end{array}\right.
$$

The following picture represents $\alpha$ 's sequential best reply for each possible

subgame at stage 4 when $\theta \in\left[\frac{1}{2}, 1\right]$

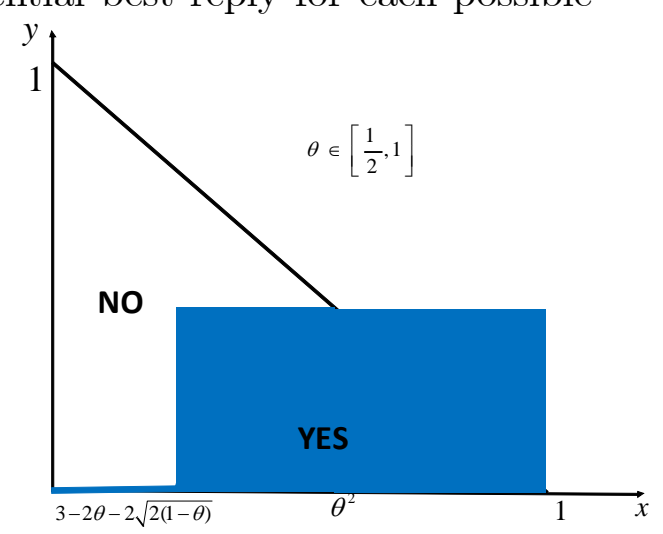


Step 3 In any second stage subgame $H^{2}, \beta$ chooses $y$ to maximize his utility subject to $S B R^{\alpha}\left(H^{3}\right)$. Again, we distinguish two different set of subgames, i.e. whether in step $2 \beta$ rejected or accepted $\alpha$ 's proposal. If $N \in H^{2}$, then $\beta$ 's choice is irrelevant as both players will anyway get the default outcome. Hence

$$
\forall H^{2} \text { s.t. } N \in H^{2} \quad S B R^{\beta}\left(H^{2}\right) \in[0,1],
$$

otherwise

$$
\forall H^{2} \text { s.t. } Y \in H^{2} \quad S B R^{\beta}\left(H^{2}\right) \in \operatorname{argmax}_{y} U^{\beta}\left(h, y ; S B R^{\alpha}\left(H^{3}\right) \mid H^{2}\right) .
$$

To solve this maximization problem, note that $\beta$ can always guarantee itself the reservation payoff $\theta$ by refusing any proposal. Otherwise, we have a maximization problem depending on $\theta$ and on the value of $x$ that characterizes subgame $H^{2}$. Moreover, note that $\beta$ objective function $\left(\frac{1}{2}-\frac{1}{2} x-\frac{1}{2} y+\sqrt{y}\right)$ has an unconstrained maximum in $y=1$ which is

i. greater than $1-x$ for any $x \in[0,1]$ and for any $\theta \in[0,1]$,

ii. greater or smaller than $1-x+2 \sqrt{x}-2 \theta$ depending on $x$ and $\theta$ :

$$
\begin{gathered}
\theta \in\left[0, \frac{1}{2}\right] \Rightarrow 1-x+2 \sqrt{x}-2 \theta \leq 1 \Leftrightarrow x \leq 2(1-\theta-\sqrt{1-2 \theta}) \\
\theta \in\left[\frac{1}{2}, 1\right] \Rightarrow 1-x+2 \sqrt{x}-2 \theta \leq 1 \quad \forall x \in[0,1] .
\end{gathered}
$$

Moreover, note that

$$
\forall \theta \in\left[0, \frac{1}{2}\right] \quad \theta^{2} \leq 2(1-\theta-\sqrt{1-2 \theta}) .
$$

Thus, we might conclude that

$$
\forall \theta \in[0,1] \quad \max \{1-x+2 \sqrt{x}-2 \theta, 1-x\} \leq 1 .
$$

Now, let we consider the different $\theta$ regions:

1. when $\theta \in\left[0, \frac{1}{2}\right]$ then:

(a) if $x \in\left[0, \theta^{2}\right]$, then the maximization problem is

$$
\begin{aligned}
& \max _{y}\left(\frac{1}{2}-\frac{1}{2} x-\frac{1}{2} y+\sqrt{y}\right) \\
& \text { s.t. } y \in[0,1-x+2 \sqrt{x}-2 \theta]
\end{aligned}
$$

which implies

$$
y^{*}=1-x+2 \sqrt{x}-2 \theta
$$


(b) when $x \in\left[\theta^{2}, 1\right]$, then the maximization problem is

$$
\begin{gathered}
\max _{y}\left(\frac{1}{2}-\frac{1}{2} x-\frac{1}{2} y+\sqrt{y}\right) \\
\text { s.t. } y \in[0,1-x]
\end{gathered}
$$

which implies

$$
y^{*}=1-x
$$

2. when $\theta \in\left[\frac{1}{2}, 1\right]$ then:

(a) if $x \in[0,3-2 \theta-2 \sqrt{2(1-\theta)}]$, then the maximization problem is

$$
\begin{gathered}
\max _{y}\left(\frac{1}{2}-\frac{1}{2} x-\frac{1}{2} y+\sqrt{y}\right) \\
\text { s.t. } y \in\{0\}
\end{gathered}
$$

which implies

$$
y^{*}=0
$$

(b) if $x \in\left[3-2 \theta-2 \sqrt{2(1-\theta)}, \theta^{2}\right]$, then the maximization problem is

$$
\begin{aligned}
& \max _{y}\left(\frac{1}{2}-\frac{1}{2} x-\frac{1}{2} y+\sqrt{y}\right) \\
& \text { s.t. } y \in[0,1-x+2 \sqrt{x}-2 \theta]
\end{aligned}
$$

which implies

$$
y^{*}=1-x+2 \sqrt{x}-2 \theta
$$

(c) if $x \in\left[\theta^{2}, 1\right]$, then the maximization problem is

$$
\begin{gathered}
\max _{y}\left(\frac{1}{2}-\frac{1}{2} x-\frac{1}{2} y+\sqrt{y}\right) \\
\text { s.t. } y \in[0,1-x]
\end{gathered}
$$

which implies

$$
y^{*}=1-x .
$$

Hence the local sequential best response of $\beta$ is 
1. if $\theta \in\left[0, \frac{1}{2}\right]$, then

$$
\widetilde{S B R}^{\beta}\left(H^{2}\right) \in\left\{\begin{array}{cl}
\{1-x+2 \sqrt{x}-2 \theta\} & \text { if } H^{2} \in\left\{(x, Y): x \in\left[0, \theta^{2}\right]\right. \\
\{1-x\} & \text { if } H^{2} \in\left\{(x, Y): x \in\left[\theta^{2}, 1\right]\right\} \\
{[0,1]} & \text { if } H^{2} \in\{(x, N): x \in[0,1]\}
\end{array}\right\}
$$

2. if $\theta \in\left[\frac{1}{2}, 1\right]$, then

$$
\widetilde{S B R} \beta\left(H^{2}\right) \in\left\{\begin{array}{cc}
\{0\} & \text { if } H^{2} \in\{(x, Y): x \in[0,3-2 \theta-2 \sqrt{2(1-\theta)}]\} \\
\{1-x+2 \sqrt{x}-2 \theta\} & \text { if } H^{2} \in\left\{(x, Y): x \in\left[3-2 \theta-2 \sqrt{2(1-\theta)}, \theta^{2}\right]\right\} \\
\{1-x\} & \text { if } H^{2} \in\left\{(x, Y): x \in\left[\theta^{2}, 1\right]\right\} \\
{[0,1]} & \text { if } H^{2} \in\{(x, N): x \in[0,1]\}
\end{array}\right.
$$

Now, we should check whether the local sequential best response it is also global, i.e. that:

$$
U^{\beta}\left(1-x-\widetilde{S B R}^{\beta}\left(H^{2}\right), \widetilde{S B R}^{\beta}\left(H^{2}\right) ; S B R^{\alpha}\left(H^{3}\right) \mid H^{2}\right) \geq \theta
$$

1. if $\theta \in\left[0, \frac{1}{2}\right]$, then

$$
\begin{gathered}
U^{\beta}\left(1-x-\widetilde{S B R}^{\beta}\left(H^{2}\right), \widetilde{S B R}^{\beta}\left(H^{2}\right) ; S B R^{\alpha}\left(H^{3}\right) \mid H^{2}\right)= \\
=\left\{\begin{array}{cc}
\theta-\sqrt{x}+\sqrt{1-x+2 \sqrt{x}-2 \theta} & \text { if } H^{2} \in\left\{(x, Y): x \in\left[3-2 \theta-2 \sqrt{2(1-\theta)}, \theta^{2}\right]\right\} \\
\sqrt{1-x} & \text { if } H^{2} \in\left\{(x, Y): x \in\left[\theta^{2}, 1\right]\right\} \\
\theta & \text { if } H^{2} \in\{(x, N): x \in[0,1]\}
\end{array}\right.
\end{gathered}
$$

Thus when $x \in\left[0, \theta^{2}\right]$

$$
\begin{gathered}
U^{\beta}\left(1-x-\widetilde{S B R}^{\beta}\left(H^{2}\right), \widetilde{S B R}^{\beta}\left(H^{2}\right) ; S B R^{\alpha}\left(H^{3}\right) \mid H^{2}\right) \geq \theta \Leftrightarrow \\
\Leftrightarrow \theta-\sqrt{x}+\sqrt{1-x+2 \sqrt{x}-2 \theta} \geq \theta \Leftrightarrow \sqrt{1-x+2 \sqrt{x}-2 \theta} \geq \sqrt{x} \Leftrightarrow \\
\Leftrightarrow 1-\sqrt{3-4 \theta} \leq \sqrt{x} \leq 1+\sqrt{3-4 \theta} \Leftrightarrow 1-\sqrt{3-4 \theta} \leq \sqrt{x} .
\end{gathered}
$$

Note that $\theta \in\left[0, \frac{1}{2}\right] \Leftrightarrow 1-\sqrt{3-4 \theta} \leq 0$, hence $1-\sqrt{3-4 \theta} \leq \sqrt{x}$ is always satisfied and thus

$x \in\left[3-2 \theta-2 \sqrt{2(1-\theta)}, \theta^{2}\right] \Leftrightarrow U^{\beta}\left(1-x-\widetilde{S B R}^{\beta}\left(H^{2}\right), \widetilde{S B R}^{\beta}\left(H^{2}\right) ; S B R^{\alpha}\left(H^{3}\right) \mid H^{2}\right) \geq$ 
when $x \in\left[\theta^{2}, 1\right]$

$$
U^{\beta}\left(\widetilde{S B R}^{\beta}\left(H^{2}\right)\right) \geq \theta \Leftrightarrow \sqrt{1-x} \geq \theta \Leftrightarrow 1-x \geq \theta^{2} \Leftrightarrow x \leq 1-\theta^{2} .
$$

However, note that

$$
\theta \in\left[\frac{1}{\sqrt{2}}, 1\right] \Rightarrow\left[\theta^{2}, 1-\theta^{2}\right]=\varnothing
$$

2. if $\theta \in\left[\frac{1}{2}, 1\right]$, then

$$
\begin{gathered}
U^{\beta}\left(1-x-\widetilde{S B R}^{\beta}\left(H^{2}\right), \widetilde{S B R}^{\beta}\left(H^{2}\right) ; S B R^{\alpha}\left(H^{3}\right) \mid H^{2}\right)= \\
=\left\{\begin{array}{cc}
\frac{1}{2}-\frac{1}{2} x & \text { if } H^{2} \in\{(x, Y): x \in[0,3-2 \theta-2 \sqrt{2(1-\theta)}]\} \\
\theta-\sqrt{x}+\sqrt{1-x+2 \sqrt{x}-2 \theta} & \text { if } H^{2} \in\left\{(x, Y): x \in\left[3-2 \theta-2 \sqrt{2(1-\theta)}, \theta^{2}\right]\right\} \\
\sqrt{1-x} & \text { if } H^{2} \in\left\{(x, Y): x \in\left[\theta^{2}, 1\right]\right\} \\
\theta & \text { if } H^{2} \in\{(x, N): x \in[0,1]\}
\end{array}\right.
\end{gathered}
$$

Thus when $x \in[0,3-2 \theta-2 \sqrt{2(1-\theta)}]$

$$
\begin{gathered}
U^{\beta}\left(1-x-\widetilde{S B R}^{\beta}\left(H^{2}\right), \widetilde{S B R}^{\beta}\left(H^{2}\right) ; S B R^{\alpha}\left(H^{3}\right) \mid H^{2}\right) \geq \theta \Leftrightarrow \\
\Leftrightarrow \frac{1}{2}-\frac{1}{2} x \geq \theta \Leftrightarrow x \leq 1-2 \theta .
\end{gathered}
$$

Note that $\theta \in\left[\frac{1}{2}, 1\right] \Rightarrow 1-2 \theta \leq 0$, hence

$x \in[0,3-2 \theta-2 \sqrt{2(1-\theta)}] \Leftrightarrow U^{\beta}\left(1-x-\widetilde{S B R}^{\beta}\left(H^{2}\right), \widetilde{S B R}^{\beta}\left(H^{2}\right) ; S B R^{\alpha}\left(H^{3}\right) \mid H^{2}\right) \leq \theta$

when $x \in\left[3-2 \theta-2 \sqrt{2(1-\theta)}, \theta^{2}\right]$

$$
\begin{gathered}
U^{\beta}\left(1-x-\widetilde{S B R}^{\beta}\left(H^{2}\right), \widetilde{S B R}^{\beta}\left(H^{2}\right) ; S B R^{\alpha}\left(H^{3}\right) \mid H^{2}\right) \geq \theta \Leftrightarrow \\
\Leftrightarrow \theta-\sqrt{x}+\sqrt{1-x+2 \sqrt{x}-2 \theta} \geq \theta \Leftrightarrow \sqrt{1-x+2 \sqrt{x}-2 \theta} \geq \sqrt{x} \Leftrightarrow \\
\Leftrightarrow 1-\sqrt{3-4 \theta} \leq \sqrt{x} \leq 1+\sqrt{3-4 \theta} \Leftrightarrow 1-\sqrt{3-4 \theta} \leq \sqrt{x} .
\end{gathered}
$$

Note that $\theta \in\left[\frac{1}{2}, \frac{3}{4}\right] \Leftrightarrow 1-\sqrt{3-4 \theta} \leq \sqrt{x} \Leftrightarrow x \geq 4(1-\theta)-2 \sqrt{3-4 \theta}$, hence

$$
x \in[3-2 \theta-2 \sqrt{2(1-\theta)}, 4(1-\theta)-2 \sqrt{3-4 \theta}] \Leftrightarrow
$$




$$
\begin{gathered}
\Leftrightarrow U^{\beta}\left(1-x-\widetilde{S B R}^{\beta}\left(H^{2}\right), \widetilde{S B R}^{\beta}\left(H^{2}\right) ; S B R^{\alpha}\left(H^{3}\right) \mid H^{2}\right) \leq \theta \\
x \in\left[4(1-\theta)-2 \sqrt{3-4 \theta}, \theta^{2}\right] \Leftrightarrow U^{\beta}\left(1-x-\widetilde{S B R}\left(H^{2}\right), \widetilde{S B R}\left(H^{2}\right) ; S B R^{\alpha}\left(H^{3}\right) \mid H^{2}\right) \geq \theta
\end{gathered}
$$

when $\theta \in\left[\frac{3}{4}, 1\right]$ the inequality has no solution, hence

$x \in\left[3-2 \theta-2 \sqrt{2(1-\theta)}, \theta^{2}\right] \Leftrightarrow U^{\beta}\left(1-x-\widetilde{S B R}^{\beta}\left(H^{2}\right), \widetilde{S B R}^{\beta}\left(H^{2}\right) ; S B R^{\alpha}\left(H^{3}\right) \mid H^{2}\right) \leq$

when $x \in\left[\theta^{2}, 1\right]$

$$
U^{\beta}\left(\widetilde{S B R}^{\beta}\left(H^{2}\right)\right) \geq \theta \Leftrightarrow \sqrt{1-x} \geq \theta \Leftrightarrow 1-x \geq \theta^{2} \Leftrightarrow x \leq 1-\theta^{2} .
$$

However, note that

$$
\theta \in\left[\frac{1}{\sqrt{2}}, 1\right] \Rightarrow\left[\theta^{2}, 1-\theta^{2}\right]=\varnothing
$$

Hence when $\theta \in\left[0, \frac{1}{\sqrt{2}}\right]$

$$
\begin{aligned}
& x \in\left[\theta^{2}, 1-\theta^{2}\right] \Leftrightarrow U^{\beta}\left(1-x-\widetilde{S B R}^{\beta}\left(H^{2}\right), \widetilde{S B R}^{\beta}\left(H^{2}\right) ; S B R^{\alpha}\left(H^{3}\right) \mid H^{2}\right) \geq \theta \\
& x \in\left[1-\theta^{2}, 1\right] \Leftrightarrow U^{\beta}\left(1-x-\widetilde{S B R}^{\beta}\left(H^{2}\right), \widetilde{S B R}^{\beta}\left(H^{2}\right) ; S B R^{\alpha}\left(H^{3}\right) \mid H^{2}\right) \leq \theta \\
& \text { when } \theta \in\left[\frac{1}{\sqrt{2}}, 1\right] \\
& x \in\left[\theta^{2}, 1\right] \Leftrightarrow U^{\beta}\left(1-x-\widetilde{S B R}^{\beta}\left(H^{2}\right), \widetilde{S B R}^{\beta}\left(H^{2}\right) ; S B R^{\alpha}\left(H^{3}\right) \mid H^{2}\right) \leq \theta
\end{aligned}
$$

Hence the global sequential best response of $\beta$ is:

1. $\theta \in\left[0, \frac{1}{2}\right]$

$$
S B R^{\beta}\left(H^{2}\right) \in\left\{\begin{array}{cc}
\{1-x+2 \sqrt{x}-2 \theta\} & \text { if } H^{2} \in\left\{(x, Y): x \in\left[0, \theta^{2}\right]\right\} \\
\{1-x\} & \text { if } H^{2} \in\left\{(x, Y): x \in\left[\theta^{2}, 1-\theta^{2}\right]\right\} \\
(1-x, 1] & \text { if } H^{2} \in\left\{(x, Y): x \in\left[1-\theta^{2}, 1\right]\right\} \\
{[0,1]} & \text { if } H^{2} \in\{(x, N): x \in[0,1]\}
\end{array}\right.
$$

2. $\theta \in\left[\frac{1}{2}, \frac{1}{\sqrt{2}}\right]$

$$
S B R^{\beta}\left(H^{2}\right) \in
$$




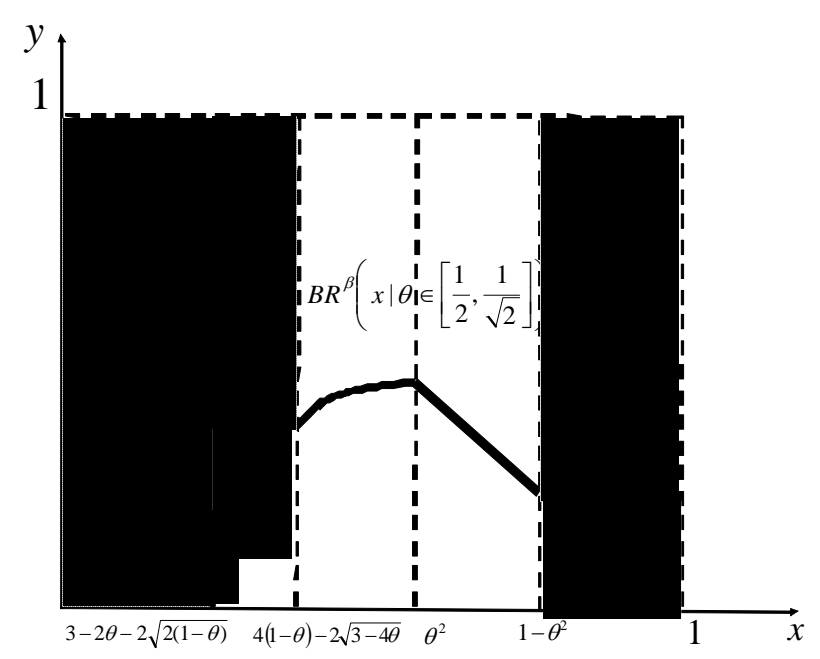

Figure 7:

$$
\in\left\{\begin{array}{cc}
H^{2} \in\{(x, Y): x \in[0,3-2 \theta-2 \sqrt{2(1-\theta)}]\} \\
(1-x+2 \sqrt{x}-2 \theta, 1] & H^{2} \in\{(x, Y): x \in[3-2 \theta-2 \sqrt{2(1-\theta)}, 4(1-\theta)-2 \sqrt{3-4 \theta}] \\
\{1-x+2 \sqrt{x}-2 \theta\} & H^{2} \in\left\{(x, Y): x \in\left[4(1-\theta)-2 \sqrt{3-4 \theta}, \theta^{2}\right]\right\} \\
\{1-x\} & H^{2} \in\left\{(x, Y): x \in\left[\theta^{2}, 1-\theta^{2}\right]\right\} \\
(1-x, 1] & H^{2} \in\left\{(x, Y): x \in\left[1-\theta^{2}, 1\right]\right\} \\
{[0,1]} & H^{2} \in\{(x, N): x \in[0,1]\}
\end{array}\right.
$$

The following picture represents the best reply correspondence for this case, the other situations are similar, hence the pictures are omitted

3. $\theta \in\left[\frac{1}{\sqrt{2}}, \frac{3}{4}\right]$

$$
\in\left\{\begin{aligned}
(0,1] & H^{2} \in\{(x, Y): x \in[0,3-2 \theta-2 \sqrt{2(1-\theta)}]\} \\
(1-x+2 \sqrt{x}-2 \theta, 1] & H^{2} \in\{(x, Y): x \in[3-2 \theta-2 \sqrt{2(1-\theta)}, 4(1-\theta)-2 \sqrt{3-4 \theta} \\
\{1-x+2 \sqrt{x}-2 \theta\} & H^{2} \in\left\{(x, Y): x \in\left[4(1-\theta)-2 \sqrt{3-4 \theta}, \theta^{2}\right]\right\} \\
(1-x, 1] & H^{2} \in\left\{(x, Y): x \in\left[\theta^{2}, 1\right]\right\} \\
{[0,1] } & H^{2} \in\{(x, N): x \in[0,1]\}
\end{aligned}\right.
$$


4. $\theta \in\left[\frac{3}{4}, 1\right]$

$$
S B R^{\beta}\left(H^{2}\right) \in\left\{\begin{array}{cc}
(0,1] & H^{2} \in\{(x, Y): x \in[0,3-2 \theta-2 \sqrt{2(1-\theta)}] \\
(1-x+2 \sqrt{x}-2 \theta, 1] & H^{2} \in\left\{(x, Y): x \in\left[3-2 \theta-2 \sqrt{2(1-\theta)}, \theta^{2}\right]\right. \\
(1-x, 1] & H^{2} \in\left\{(x, Y): x \in\left[\theta^{2}, 1\right]\right\} \\
{[0,1]} & H^{2} \in\{(x, N): x \in[0,1]\}
\end{array}\right.
$$

Step $2 \beta$ chooses whether to support $\alpha$ proposal on $x$, for any $x \in[0,1]$, anticipating $S B R^{\beta}\left(H^{2}\right)$ and $S B R^{\alpha}\left(H^{3}\right)$. In particular, suppose

$$
S B R^{\alpha}(\underbrace{x, S B R^{\beta}(x), S B R^{\beta}(\underbrace{x, S B R^{\beta}(x)}_{H^{2}})}_{H^{3}})=Y,
$$

then $\operatorname{SBR}^{\beta}(x)=Y$ if and only if

$$
\begin{gathered}
U^{\beta}\left(1-x-S B R^{\beta}\left(H^{2}\right), S B R^{\beta}\left(H^{2}\right) ; Y, S B R^{\alpha}\left(H^{3}\right)=Y \mid x\right) \geq \\
\geq U^{\beta}\left(1-x-S B R^{\beta}\left(H^{2}\right), S B R^{\beta}\left(H^{2}\right) ; N, S B R^{\alpha}\left(H^{3}\right)=Y \mid x\right)=U^{D} \Leftrightarrow \\
\Leftrightarrow x \leq 1-S B R^{\beta}\left(H^{2}\right)+2 \sqrt{S B R^{\beta}\left(H^{2}\right)}-2 \theta .
\end{gathered}
$$

Hence:

1. Suppose $\theta \in\left[0, \frac{1}{2}\right]$, then $S B R^{\beta}(x)=Y$ if and only if

$$
\begin{aligned}
& x \leq 1-S B R^{\beta}\left(H^{2}\right)+2 \sqrt{S B R^{\beta}\left(H^{2}\right)}-2 \theta \Leftrightarrow \\
& \Leftrightarrow\left\{\begin{array}{cr}
x \leq 1-(1-x+2 \sqrt{x}-2 \theta)+2 \sqrt{1-x+2 \sqrt{x}-2 \theta}-2 \theta & \text { and } x \in\left[0, \theta^{2}\right] \\
x \leq 1-(1-x)+2 \sqrt{1-x}-2 \theta & \text { and } x \in\left[\theta^{2}, 1-\theta^{2}\right]
\end{array} \Leftrightarrow\right. \\
& \Leftrightarrow\left\{\begin{array}{c}
2 x-2 \sqrt{x}-(1-2 \theta) \leq 0 \quad \text { and } \quad x \in\left[0, \theta^{2}\right] \\
x \leq 1-\theta^{2} \quad \text { and } x \in\left[\theta^{2}, 1-\theta^{2}\right]
\end{array} \Leftrightarrow\right. \\
& \Leftrightarrow\left\{\begin{array}{c}
x \in\left[0, \theta^{2}\right] \\
x \in\left[\theta^{2}, 1-\theta^{2}\right] .
\end{array}\right.
\end{aligned}
$$

Therefore when $\theta \in\left[0, \frac{1}{2}\right]$

$$
\operatorname{SBR}^{\beta}(x)=\left\{\begin{array}{lll}
Y & \text { if } & x \in\left[0,1-\theta^{2}\right] \\
N & \text { if } & x \in\left[1-\theta^{2}, 1\right]
\end{array} .\right.
$$


2. Suppose $\theta \in\left[\frac{1}{2}, \frac{1}{\sqrt{2}}\right]$, then $S B R^{\beta}(x)=Y$ if and only if

$$
\begin{aligned}
& x \leq 1-S B R^{\beta}\left(H^{2}\right)+2 \sqrt{S B R^{\beta}\left(H^{2}\right)}-2 \theta \Leftrightarrow
\end{aligned}
$$

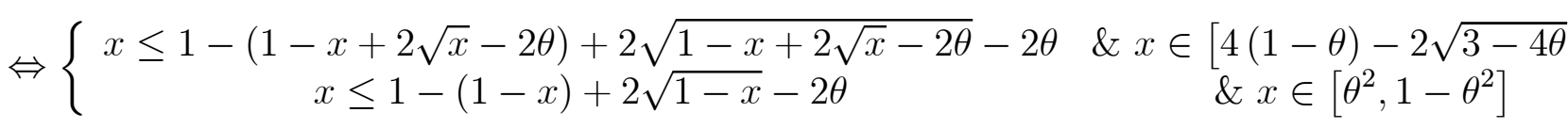

$$
\begin{aligned}
& \Leftrightarrow\left\{\begin{array}{cc}
2 x-2 \sqrt{x}-(1-2 \theta) \leq 0 & \text { and } x \in\left[4(1-\theta)-2 \sqrt{3-4 \theta}, \theta^{2}\right] \\
x \leq 1-\theta^{2} & \text { and } x \in\left[\theta^{2}, 1-\theta^{2}\right]
\end{array} \Leftrightarrow\right. \\
& \Leftrightarrow\left\{\begin{array}{c}
x \in\left[4(1-\theta)-2 \sqrt{3-4 \theta}, \theta^{2}\right] \\
x \in\left[\theta^{2}, 1-\theta^{2}\right] .
\end{array}\right.
\end{aligned}
$$

Therefore when $\theta \in\left[\frac{1}{2}, \frac{1}{\sqrt{2}}\right]$

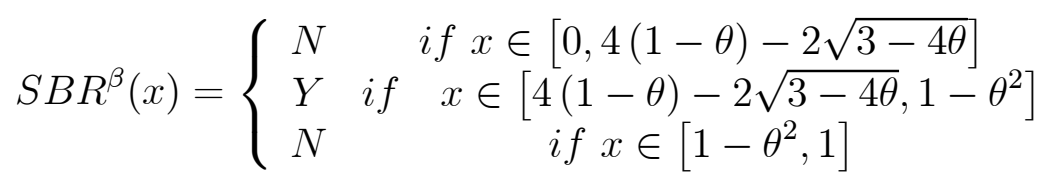

3. suppose $\theta \in\left[\frac{1}{\sqrt{2}}, \frac{3}{4}\right]$, then $S B R^{\beta}(x)=Y$ if and only if

$$
\begin{gathered}
x \leq 1-S B R^{\beta}\left(H^{2}\right)+2 \sqrt{S B R^{\beta}\left(H^{2}\right)}-2 \theta \Leftrightarrow \\
\Leftrightarrow x \leq 1-(1-x+2 \sqrt{x}-2 \theta)+\sqrt{1-x+2 \sqrt{x}-2 \theta}-2 \theta \quad \& x \in\left[4(1-\theta)-2 \sqrt{3-4 \theta}, \theta^{2}\right. \\
\Leftrightarrow 2 x-2 \sqrt{x}-(1-2 \theta) \leq 0 \text { and } x \in\left[4(1-\theta)-2 \sqrt{3-4 \theta}, \theta^{2}\right] \Leftrightarrow
\end{gathered}
$$

hence, using the calculations of step 3

$$
\Leftrightarrow x \in\left[4(1-\theta)-2 \sqrt{3-4 \theta}, \theta^{2}\right]
$$

Therefore when $\theta \in\left[\frac{1}{\sqrt{2}}, \frac{3}{4}\right]$

$$
\operatorname{SBR}^{\beta}(x)=\left\{\begin{array}{ccc}
N & \text { if } & x \in[0,4(1-\theta)-2 \sqrt{3-4 \theta}] \\
Y & \text { if } & x \in\left[4(1-\theta)-2 \sqrt{3-4 \theta}, \theta^{2}\right] \\
N & \text { if } x \in\left[\theta^{2}, 1\right] .
\end{array}\right.
$$

4. suppose $\theta \in\left[\frac{3}{4}, 1\right]$, then

$$
S_{B R} \beta(x) \in\{Y, N\} \forall x \in[0,1]
$$

because if $S B R^{\beta}(x)=Y$ then $S B R^{\beta}\left(H^{2}\right) \in(1-x+4 \sqrt{x}-4 \theta, 1]$ and then $S B R^{\alpha}\left(H^{3}\right)=N$, if $S B R^{\beta}(x)=N$ then $S B R^{\beta}\left(H^{2}\right) \in[0,1]$ and then $S B R^{\alpha}\left(H^{3}\right) \in\{Y, N\}$, however in both case

$$
U^{\beta}\left(1-x-S B R^{\beta}\left(H^{2}\right), S B R^{\beta}\left(H^{2}\right) ; Y, S B R^{\alpha}\left(H^{3}\right) \mid x\right)=U^{D} .
$$




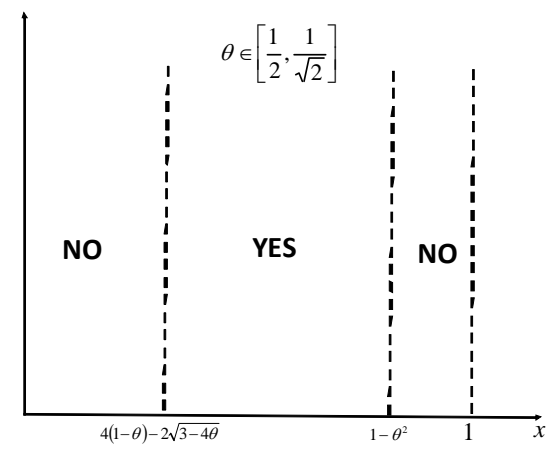

Figure 8:

Then, we might sum $S B R^{\beta}(x)$ as follows:

1. when $\theta \in\left[0, \frac{1}{2}\right]$, then

$$
S B R^{\beta}(x)=\left\{\begin{array}{lll}
Y & \text { if } & x \in\left[0,1-\theta^{2}\right] \\
N & \text { if } & x \in\left[1-\theta^{2}, 1\right] .
\end{array}\right.
$$

2. when $\theta \in\left[\frac{1}{2}, \frac{1}{\sqrt{2}}\right]$, then

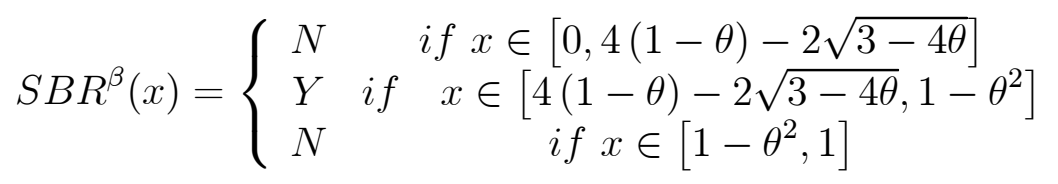

The following picture represents the best reply correspondence for this case, the other situations are similar, hence the pictures are omitted

3. when $\theta \in\left[\frac{1}{\sqrt{2}}, \frac{3}{4}\right]$, then

$$
\operatorname{SBR}^{\beta}(x)=\left\{\begin{array}{ccc}
N & \text { if } & x \in[0,4(1-\theta)-2 \sqrt{3-4 \theta}] \\
Y & \text { if } & x \in\left[4(1-\theta)-2 \sqrt{3-4 \theta}, \theta^{2}\right] \\
N & \text { if } x \in\left[\theta^{2}, 1\right] .
\end{array}\right.
$$

4. when $\theta \in\left[\frac{3}{4}, 1\right]$, then

$$
S B R^{\beta}(x) \in\{Y, N\} \forall x \in[0,1]
$$

Step $1 \alpha$ chooses $x \in[0,1]$ to maximize

$$
U^{\alpha}\left(1-x-S B R^{\beta}\left(H^{2}\right), x ; S B R^{\beta}(x), S B R^{\alpha}\left(H^{3}\right)\right)
$$

that we will denote by $U^{\alpha}(x \mid \theta)$ to simplify 
1. when $\theta \in\left[0, \frac{1}{2}\right]$, then

$$
\begin{aligned}
& U^{\alpha}(x \mid \theta)=\left\{\begin{array}{cl}
\frac{1}{2}[1-x-\underbrace{(1-x+2 \sqrt{x}-2 \theta)}_{S B R^{\beta}\left(H^{2}\right)}]+\sqrt{x} & \text { if } x \in\left[0, \theta^{2}\right] \\
\frac{1}{2}[1-x-\underbrace{(1-x)}_{S B R^{\beta}\left(H^{2}\right)}]+\sqrt{x} & \text { if } x \in\left[\theta^{2}, 1-\theta^{2}\right] \\
\theta & \text { if } x \in\left[1-\theta^{2}, 1\right]
\end{array}=\right. \\
& =\left\{\begin{array}{cl}
-\sqrt{x}+\theta+\sqrt{x}=\theta & \text { if } x \in\left[0, \theta^{2}\right] \\
\sqrt{x} & \text { if } x \in\left[\theta^{2}, 1-\theta^{2}\right] \\
\theta & \text { if } x \in\left[1-\theta^{2}, 1\right] .
\end{array}\right.
\end{aligned}
$$

Hence

$$
\max _{x \in[0,1]} U^{\alpha}(x \mid \theta)=\left\{\begin{array}{cc}
\sqrt{1-\theta^{2}} & \text { if } x=1-\theta^{2} \\
\theta & \text { if } x \in\left[0, \theta^{2}\right] \cup\left[1-\theta^{2}, 1\right]
\end{array}\right.
$$

since

$$
\theta \in\left[0, \frac{1}{2}\right] \Rightarrow \sqrt{1-\theta^{2}} \geq \theta
$$

then

$$
\theta \in\left[0, \frac{1}{2}\right] \Rightarrow \underset{x \in[0,1]}{\arg \max } U^{\alpha}(x \mid \theta)=1-\theta^{2}
$$

Thus when $\theta \in\left[0, \frac{1}{2}\right]$ the subgame perfect equilibrium outcome is

$$
\left(h^{\alpha L}, x^{\alpha L}, y^{\alpha L}\right)=\left(0,1-\theta^{2}, \theta^{2}\right) .
$$

2. when $\theta \in\left[\frac{1}{2}, \frac{1}{\sqrt{2}}\right]$, then

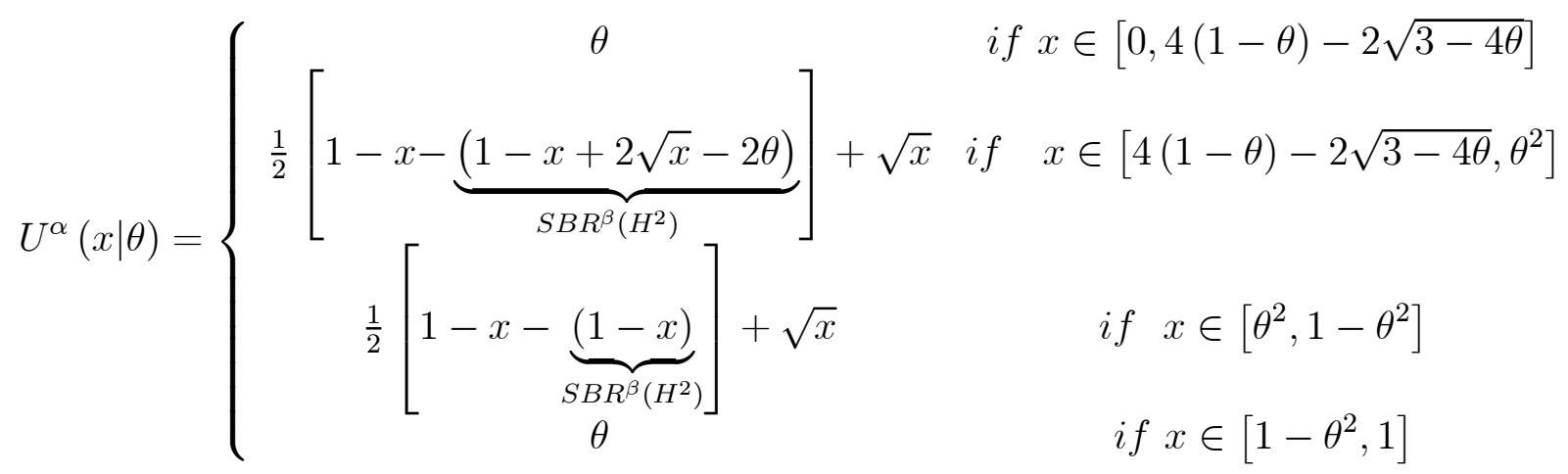




$$
U^{\alpha}(x \mid \theta)=\left\{\begin{array}{cc}
\theta & \text { if } x \in[0,4(1-\theta)-2 \sqrt{3-4 \theta}] \\
-\sqrt{x}+\theta+\sqrt{x}=\theta & \text { if } \quad x \in\left[4(1-\theta)-2 \sqrt{3-4 \theta}, \theta^{2}\right] \\
\sqrt{x} & \text { if } x \in\left[\theta^{2}, 1-\theta^{2}\right] \\
\theta & \text { if } x \in\left[\theta^{2}, 1\right] .
\end{array}\right.
$$

Hence

$$
\max _{x \in[0,1]} U^{\alpha}(x \mid \theta)=\left\{\begin{array}{cc}
\sqrt{1-\theta^{2}} & \text { if } x=1-\theta^{2} \\
\theta & \text { if } x \in\left[0, \theta^{2}\right] \cup\left[1-\theta^{2}, 1\right]
\end{array}\right.
$$

since

$$
\theta \in\left[\frac{1}{2}, \frac{1}{\sqrt{2}}\right] \Rightarrow \sqrt{1-\theta^{2}} \geq \theta
$$

then

$$
\theta \in\left[\frac{1}{2}, \frac{1}{\sqrt{2}}\right] \Rightarrow \underset{x \in[0,1]}{\arg \max } U^{\alpha}(x \mid \theta)=1-\theta^{2}
$$

Thus when $\theta \in\left[\frac{1}{2}, \frac{1}{\sqrt{2}}\right]$ the subgame perfect equilibrium outcome is

$$
\left(h^{\alpha L}, x^{\alpha L}, y^{\alpha L}\right)=\left(0,1-\theta^{2}, \theta^{2}\right) .
$$

3. when $\theta \in\left[\frac{1}{\sqrt{2}}, \frac{3}{4}\right]$, then

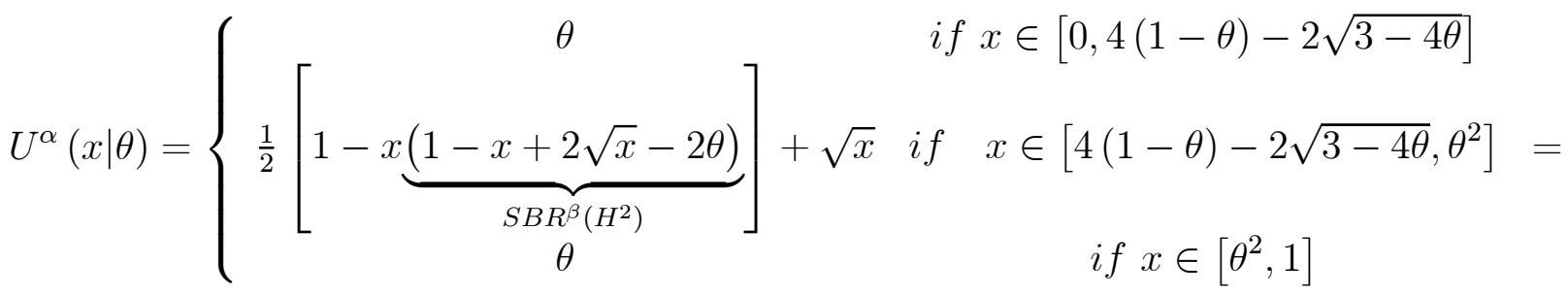

$$
\begin{aligned}
& U^{\alpha}(x \mid \theta)=\left\{\begin{array}{ccc}
\theta & \text { if } x \in[0,4(1-\theta)-2 \sqrt{3-4 \theta}] \\
-\sqrt{x}+\theta+\sqrt{x}=\theta & \text { if } & x \in\left[4(1-\theta)-2 \sqrt{3-4 \theta}, \theta^{2}\right] \\
\theta & \text { if } x \in\left[\theta^{2}, 1\right] .
\end{array}\right.
\end{aligned}
$$

Hence

$$
\max _{x \in[0,1]} U^{\alpha}(x \mid \theta)=\left\{\begin{array}{lll}
\theta & \text { if } & x \in[0,1]
\end{array}\right.
$$

then

$$
\theta \in\left[\frac{1}{\sqrt{2}}, \frac{3}{4}\right] \Rightarrow \underset{x \in[0,1]}{\arg \max } U^{\alpha}(x \mid \theta)=[0,1] .
$$

Thus when $\theta \in\left[\frac{1}{\sqrt{2}}, \frac{3}{4}\right]$ we have a continuum of outcome equivalent subgame perfect equilibria, that we denote as default outcomes:

$$
\left(h^{\alpha L}, x^{\alpha L}, y^{\alpha L}\right)=\left(h^{D}, x^{D}, y^{D}\right)
$$


4. when $\theta \in\left[\frac{3}{4}, 1\right]$, then

$$
U^{\alpha}(x \mid \theta)= \begin{cases}\theta & \text { if } x \in[0,1]=\end{cases}
$$

Hence

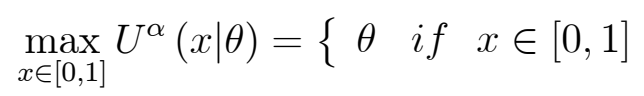

then

$$
\theta \in\left[\frac{3}{4}, 1\right] \Rightarrow \underset{x \in[0,1]}{\arg \max } U^{\alpha}(x \mid \theta)=[0,1] .
$$

In, this case either $\alpha$ or $\beta$ would refuse the logroll and the unique subgame perfect equilibrium outcome is

$$
\left(h^{\alpha L}, x^{\alpha L}, y^{\alpha L}\right)=\left(h^{D}, x^{D}, y^{D}\right) .
$$

To conclude, the subgame perfect equilibrium outcomes of the logrolling game are

$$
\left(h^{\alpha L}, x^{\alpha L}, y^{\alpha L}\right)=\left\{\begin{array}{cl}
\left(0,1-\theta^{2}, \theta^{2}\right) & \text { if } \theta \in\left[0, \frac{1}{\sqrt{2}}\right] \\
\left(h^{D}, x^{D}, y^{D}\right) & \text { if } \theta \in\left[\frac{1}{\sqrt{2}}, 1\right] .
\end{array}\right.
$$

\subsubsection{Example 2: Stone Geary Payoff Functions}

The equilibrium policy outcome is calculated by backward induction.

Step $4 \alpha$ chooses between to accept the policy proposed by $\beta$ and the default policy. Let distinguish two different sets of third stage subgames $H^{3}$, whether in step $2 \beta$ rejected or accepted $\alpha$ 's proposal. If $N \in H^{3}$, then $\alpha$ 's choice is irrelevant as both players will get the default outcome. Hence $\alpha$ sequential best reply at $H^{3}$ is

$$
\forall H^{3} \text { s.t. } N \in H^{3} \quad S B R^{\alpha}\left(H^{3}\right) \in\{Y, N\} .
$$

On the other hand, if $Y \in H^{3}$, then

$$
S B R^{\alpha}\left(H^{3}\right)=Y \Leftrightarrow U^{\alpha}\left(1-x-y, y ; Y \mid H^{3}\right) \geq U^{D}
$$

and, using the resource constraint, which is binding since $U^{\alpha}$ is increasing in $(h, x)$

$$
h+x+y=1,
$$

then

$\Leftrightarrow \log (1-x-y+1)+2 \log (x+1) \geq \theta \Leftrightarrow(1-x-y+1)(x+1)^{2} \geq e^{\theta} \Leftrightarrow$ 


$$
\Leftrightarrow(2-x-y) \geq \frac{e^{\theta}}{(x+1)^{2}} \Leftrightarrow 0 \leq \widetilde{y} \leq \min \left\{2-x-\frac{e^{\theta}}{(x+1)^{2}}, 1-x\right\} .
$$

Note that

$$
2-x-\frac{e^{\theta}}{(x+1)^{2}} \leq 1-x \Leftrightarrow x \in\left[0, \sqrt{e^{\theta}}-1\right]
$$

and that

$$
\forall \theta \in[0, \ln 2] \quad\left\{2-x-\frac{e^{\theta}}{(x+1)^{2}}, 1-x\right\} \geq 0
$$

while

$$
\forall \theta \in[\ln 2, \ln 4] \quad 2-x-\frac{e^{\theta}}{(x+1)^{2}} \geq 0 \Leftrightarrow x \in\left[a(\theta), \sqrt{e^{\theta}}-1\right]
$$

where $a(\theta)$ is the solution of $2-x-\frac{e^{\theta}}{(x+1)^{2}}=0$ in $x \in\left[0, \sqrt{e^{\theta}}-1\right]$ when $\theta \in[\ln 2, \ln 4]$, which is possible to show that exists ${ }^{18}$. Hence, we can write $\alpha$ 's "incentive logrolling constraint" (IL SG-1) for our Stone Geary payoffs as follows

$$
\begin{aligned}
& S B R^{\alpha}\left(H^{3}\right)=Y \Leftrightarrow U^{\alpha}\left(1-x-y, y ; Y \mid H^{3}\right) \geq U^{D} \Leftrightarrow \\
& \theta \in[0, \ln 2] \Rightarrow y \in\left\{\begin{array}{ccc}
{\left[0,2-x-\frac{e^{\theta}}{(x+1)^{2}}\right]} & \text { if } x \in\left[0, \sqrt{e^{\theta}}-1\right. \\
{[0,1-x]} & \text { if } x \in\left[\sqrt{e^{\theta}}-1,1\right.
\end{array}\right] \\
& \theta \in[\ln 2, \ln 4] \Rightarrow y \in\left\{\begin{array}{cc}
\{0\} & \text { if } x \in[0, a(\theta)] \\
{\left[0,2-x-\frac{e^{\theta}}{(x+1)^{2}}\right]} & \text { if } x \in\left[a(\theta), \sqrt{e^{\theta}}-1\right] \\
{[0,1-x]} & \text { if } x \in\left[\sqrt{e^{\theta}}-1,1\right]
\end{array}\right.
\end{aligned}
$$

Hence, we get the following sequential best reply for $\alpha$ :

$$
\begin{gathered}
\theta \in[0, \ln 2] \Rightarrow S B R^{\alpha}\left(H^{3}\right) \in \\
\in\left\{\begin{array}{cc}
\{Y, N\} & H^{3} \in\{(x, N, y):(x, y) \in[0,1] \times[0,1]\} \\
\{Y\} & H^{3} \in\left\{(x, Y, y):(x, y) \in\left[0, \sqrt{e^{\theta}}-1\right] \times\left[0,2-x-\frac{e^{\theta}}{(x+1)^{2}}\right]\right\} \\
\{N\} & H^{3} \in\left\{(x, Y, y):(x, y) \in\left[0, \sqrt{e^{\theta}}-1\right] \times\left(2-x-\frac{e^{\theta}}{(x+1)^{2}}, 1\right]\right\} \\
\{Y\} & H^{3} \in\left\{(x, Y, y):(x, y) \in\left[\sqrt{e^{\theta}}-1,1\right] \times[0,1-x]\right\} .
\end{array}\right.
\end{gathered}
$$

${ }^{18}$ The graphical analysis shows that there exists an intersection of with the $x$ axis, while Maple gives $\frac{1}{\sqrt[3]{-\frac{1}{2} e^{\theta}+\sqrt{-e^{\theta}+\frac{1}{4} e^{2 \theta}}+1}}+\sqrt[3]{-\frac{1}{2} e^{\theta}+\sqrt{-e^{\theta}+\frac{1}{4} e^{2 \theta}}+1}$ as solution. 


$$
\begin{gathered}
\theta \in[\ln 2, \ln 4] \Rightarrow S B R^{\alpha}\left(H^{3}\right) \in \\
\in\left\{\begin{array}{cc}
\{Y, N\} & H^{3} \in\{(x, N, y):(x, y) \in[0,1] \times[0,1]\} \\
\{Y\} & H^{3} \in\{(x, Y, y):(x, y) \in[0, a(\theta)] \times\{0\}\} \\
\{N\} & H^{3} \in\{(x, Y, y):(x, y) \in[0, a(\theta)] \times(0,1]\} \\
\{Y\} & H^{3} \in\left\{(x, Y, y):(x, y) \in\left[a(\theta), \sqrt{e^{\theta}}-1\right] \times\left[0,2-x-\frac{e^{\theta}}{(x+1)^{2}}\right]\right\} \\
\{N\} & H^{3} \in\left\{(x, Y, y):(x, y) \in\left[a(\theta), \sqrt{e^{\theta}}-1\right] \times\left(2-x-\frac{e^{\theta}}{(x+1)^{2}}, 1-x\right]\right\} \\
\{Y\} & H^{3} \in\left\{(x, Y, y):(x, y) \in\left[\sqrt{e^{\theta}}-1,1\right] \times[0,1-x]\right\} .
\end{array}\right.
\end{gathered}
$$

Step 3 In any second stage subgame $H^{2}, \beta$ chooses $y$ to maximize his utility subject to $S B R^{\alpha}\left(H^{3}\right)$. Again, we distinguish two different set of subgames, i.e. whether in step $2 \beta$ rejected or accepted $\alpha$ 's proposal. If $N \in H^{2}$, then $\beta$ 's choice is irrelevant as both players will anyway get the default outcome. Hence

$$
\forall H^{2} \quad \text { s.t. } N \in H^{2} \quad S B R^{\beta}\left(H^{2}\right) \in[0,1],
$$

otherwise

$$
\forall H^{2} \quad \text { s.t. } Y \in H^{2} \quad S B R^{\beta}\left(H^{2}\right) \in \operatorname{argmax}_{y} U^{\beta}\left(h, y ; S B R^{\alpha}\left(H^{3}\right) \mid H^{2}\right) .
$$

To solve this maximization problem, note that $\beta$ can always guarantee itself the reservation payoff $\theta$ by refusing any proposal. Otherwise, we have a maximization problem depending on $\theta$ and on the value of $x$ that characterizes subgame $H^{2}$. Moreover, note that $\beta$ objective function has an unconstrained maximum in $y=1-\frac{2}{3} x$ which is greater than $1-x$ for any $x \in[0,1]$ and for any $\theta \in[0, \ln 4]$, hence is also greater than $\min \left\{2-x-\frac{e^{\theta}}{(x+1)^{2}}, 1-x\right\}$. Then $\beta$ maximization problem depends on the range of $\theta$ and of $x$ :

1. when $\theta \in[0, \ln 2]$

(a) if $x \in\left[0, \sqrt{e^{\theta}}-1\right]$, then the maximization problem is

$$
\begin{gathered}
\max _{y}(\ln (1+1-x-y)+2 \ln (1+y)) \\
\text { s.t. } y \in\left[0,2-x-\frac{e^{\theta}}{(x+1)^{2}}\right]
\end{gathered}
$$

which implies

$$
y^{*}=2-x-\frac{e^{\theta}}{(x+1)^{2}}
$$


(b) If $x \in\left[\sqrt{e^{\theta}}-1,1\right]$, then the maximization problem is

$$
\begin{gathered}
\max _{y}(\ln (1+1-x-y)+2 \ln (1+y)) \\
\text { s.t. } y \in[0,1-x]
\end{gathered}
$$

which implies

$$
y^{*}=1-x
$$

2. when $\theta \in[\ln 2, \ln 4]$

(a) If $x \in[0, a(\theta)]$, then the maximization problem is

$$
\begin{gathered}
\max _{y}(\ln (1+1-x-y)+2 \ln (1+y)) \\
\text { s.t. } y \in\{0\}
\end{gathered}
$$

which implies

$$
y^{*}=0
$$

(b) If $x \in\left[a(\theta), \sqrt{e^{\theta}}-1\right]$, then the maximization problem is

$$
\begin{gathered}
\max _{y}(\ln (1+1-x-y)+2 \ln (1+y)) \\
\text { s.t. } y \in\left[a(\theta), 2-x-\frac{e^{\theta}}{(x+1)^{2}}\right]
\end{gathered}
$$

which implies

$$
y^{*}=2-x-\frac{e^{\theta}}{(x+1)^{2}}
$$

(c) If $x \in\left[\sqrt{e^{\theta}}-1,1\right]$, then the maximization problem is

$$
\begin{gathered}
\max _{y}(\ln (1+1-x-y)+2 \ln (1+y)) \\
\text { s.t. } y \in[0,1-x]
\end{gathered}
$$

which implies

$$
y^{*}=1-x .
$$

Hence the local sequential best response of $\beta$ is 
1. when $\theta \in[0, \ln 2]$

$$
\widetilde{S B R}^{\beta}\left(H^{2}\right) \in\left\{\begin{array}{cc}
\left\{2-x-\frac{e^{\theta}}{(x+1)^{2}}\right\} & \text { if } H^{2} \in\left\{(x, Y): x \in\left[0, \sqrt{e^{\theta}}-1\right]\right. \\
\{1-x\} & \text { if } H^{2} \in\left\{(x, Y): x \in\left[\sqrt{e^{\theta}}-1,1\right]\right. \\
{[0,1]} & \text { if } H^{2} \in\{(x, N): x \in[0,1]\}
\end{array}\right\}
$$

2. when $\theta \in[\ln 2, \ln 4]$

$$
\widetilde{S B R} \beta\left(H^{2}\right) \in\left\{\begin{array}{cc}
\{0\} & \text { if } H^{2} \in\{(x, Y): x \in[0, a(\theta)]\} \\
\left\{2-x-\frac{e^{\theta}}{(x+1)^{2}}\right\} & \text { if } H^{2} \in\left\{(x, Y): x \in\left[a(\theta), \sqrt{e^{\theta}}-1\right]\right. \\
\{1-x\} & \text { if } H^{2} \in\left\{(x, Y): x \in\left[\sqrt{e^{\theta}}-1,1\right]\right\} \\
{[0,1]} & \text { if } H^{2} \in\{(x, N): x \in[0,1]\}
\end{array}\right\}
$$

Now, we should check whether the local sequential best response it is also global, i.e. that:

$$
\begin{aligned}
& \theta \in[0, \ln 2] \Rightarrow U^{\beta}\left(1-x-\widetilde{S B R}^{\beta}\left(H^{2}\right), \widetilde{S B R}^{\beta}\left(H^{2}\right) ; S B R^{\alpha}\left(H^{3}\right) \mid H^{2}\right) \geq \theta \Leftrightarrow \\
& \Leftrightarrow\left\{\begin{array}{cc}
\ln \left(2-x-\left(2-x-\frac{e^{\theta}}{(x+1)^{2}}\right)\right)+ & \text { if } H^{2} \in\left\{(x, Y): x \in\left[0, \sqrt{e^{\theta}}-1\right]\right\} \\
+2 \ln \left(1+\left(2-x-\frac{e^{\theta}}{(x+1)^{2}}\right)\right) \geq \theta & \text { if } H^{2} \in\left\{(x, Y): x \in\left[\sqrt{e^{\theta}}-1,1\right]\right\} \\
2 \ln (2-x) \geq \theta & \text { if } H^{2} \in\{(x, N): x \in[0,1]\} \\
\theta \geq \theta & \Leftrightarrow
\end{array} \Leftrightarrow\right. \\
& \Leftrightarrow\left\{\begin{array}{cc}
2-2 x \geq \frac{e^{\theta}}{(x+1)^{2}} \quad \text { if } H^{2} \in\left\{(x, Y): x \in\left[0, \sqrt{e^{\theta}}-1\right]\right. \\
x \leq 2-\sqrt{e^{\theta}} \quad \text { if } H^{2} \in\left\{(x, Y): x \in\left[\sqrt{e^{\theta}}-1,1\right]\right. \\
\theta \geq \theta & \text { if } H^{2} \in\{(x, N): x \in[0,1]\}
\end{array}\right\}
\end{aligned}
$$

since

$$
\theta \in[0, \ln 2] \Leftrightarrow 2\left(1-x^{2}\right)(1+x) \geq e^{\theta} \quad \forall x \in\left[0, \sqrt{e^{\theta}}-1\right]
$$

and

$$
\theta \in[0, \ln 2] \Leftrightarrow \sqrt{e^{\theta}}-1 \leq 2-\sqrt{e^{\theta}}
$$

the global sequential best response of $\beta$ when $\theta \in[0, \ln 2]$ is:

$$
S B R^{\beta}\left(H^{2}\right) \in\left\{\begin{array}{cc}
\left\{2-x-\frac{e^{\theta}}{(x+1)^{2}}\right\} & \text { if } H^{2} \in\left\{(x, Y): x \in\left[0, \sqrt{e^{\theta}}-1\right]\right\} \\
\{1-x\} & \text { if } H^{2} \in\left\{(x, Y): x \in\left[\sqrt{e^{\theta}}-1,2-\sqrt{e^{\theta}}\right]\right\} \\
\left(2-x-\frac{e^{\theta}}{(x+1)^{2}}, 1\right] & \text { if } H^{2} \in\left\{(x, Y): x \in\left[2-\sqrt{e^{\theta}}, 1\right]\right\} \\
{[0,1]} & \text { if } H^{2} \in\{(x, N): x \in[0,1]\} .
\end{array}\right.
$$


On the other hand, suppose $\theta \in[\ln 2, \ln 4]$ then

$$
\begin{gathered}
U^{\beta}\left(1-x-\widetilde{S B R}^{\beta}\left(H^{2}\right), \widetilde{S B R}^{\beta}\left(H^{2}\right) ; S B R^{\alpha}\left(H^{3}\right) \mid H^{2}\right) \geq \theta \Leftrightarrow \\
\Leftrightarrow\left\{\begin{array}{cc}
\ln (2-x-0)+2 \ln (1+0) \geq \theta & \text { if } H^{2} \in\{(x, Y): x \in[0, a(\theta)]\} \\
\ln \left(2-x-\left(2-x-\frac{e^{\theta}}{(x+1)^{2}}\right)\right)+ & \text { if } H^{2} \in\left\{(x, Y): x \in\left[a(\theta), \sqrt{e^{\theta}}-1\right]\right\} \\
+2 \ln \left(1+\left(2-x-\frac{e^{\theta}}{(x+1)^{2}}\right)\right) \geq \theta & \text { if } H^{2} \in\left\{(x, Y): x \in\left[\sqrt{e^{\theta}}-1,1\right]\right\} \\
2 \ln (2-x) \geq \theta & \text { if } H^{2} \in\{(x, N): x \in[0,1]\} \\
\theta \geq \theta & \text { if } H^{2} \in\{(x, Y): x \in[0, a(\theta)]\} \\
\Leftrightarrow & \left\{\begin{array}{cc}
x \leq 2-e^{\theta} & \text { if } H^{2} \in\left\{(x, Y): x \in\left[a(\theta), \sqrt{e^{\theta}}-1\right]\right\} \\
2(1-x)(x+1)^{2} \geq e^{\theta} & \text { if } H^{2} \in\left\{(x, Y): x \in\left[\sqrt{e^{\theta}}-1,1\right]\right.
\end{array}\right\} \\
x \leq 2-\sqrt{e^{\theta}} & \text { if } H^{2} \in\{(x, N): x \in[0,1]\}
\end{array}\right]
\end{gathered}
$$

Since

$$
\begin{gathered}
\theta \in[\ln 2, \ln 4] \Rightarrow 2-e^{\theta} \leq 0 \\
\theta \in[\ln 2, \ln 4] \Rightarrow 2-\sqrt{e^{\theta}} \leq \sqrt{e^{\theta}}-1
\end{gathered}
$$

$\theta \in\left[\ln 2, \ln \frac{9}{4}\right] \Rightarrow 2(1-x)(x+1)^{2} \geq e^{\theta} \Leftrightarrow x \in\left[b(\theta), \sqrt{e^{\theta}}-1\right]$ where $b(\theta) \geq a(\theta)$ $\theta \in\left[\ln \frac{9}{4}, \simeq \ln 2.37\right] \Rightarrow 2(1-x)(x+1)^{2} \geq e^{\theta} \Leftrightarrow x \in[b(\theta), c(\theta)] \subset\left[a(\theta), \sqrt{e^{\theta}}-1\right]$

$$
\theta \in[\simeq \ln 2.37, \ln 4] \Rightarrow 2(1-x)(x+1)^{2} \geq e^{\theta} \Leftrightarrow x \in \varnothing
$$

where $\ln 2.37$ is the approximate solution of the equation $b(\theta)=c(\theta)$. Then we might conclude that the global sequential best response of $\beta$ is:

1. $\theta \in[0, \ln 2]$

$$
S B R^{\beta}\left(H^{2}\right) \in\left\{\begin{array}{cc}
\left\{2-x-\frac{e^{\theta}}{(x+1)^{2}}\right\} & \text { if } H^{2} \in\left\{(x, Y): x \in\left[0, \sqrt{e^{\theta}}-1\right]\right\} \\
\{1-x\} & \text { if } H^{2} \in\left\{(x, Y): x \in\left[\sqrt{e^{\theta}}-1,2-\sqrt{e^{\theta}}\right]\right\} \\
(1-x, 1] & \text { if } H^{2} \in\left\{(x, Y): x \in\left[2-\sqrt{e^{\theta}}, 1\right]\right\} \\
{[0,1]} & \text { if } H^{2} \in\{(x, N): x \in[0,1]\} .
\end{array}\right.
$$


2. $\theta \in\left[\ln 2, \ln \frac{9}{4}\right]$

$$
S B R^{\beta}\left(H^{2}\right) \in\left\{\begin{array}{cc}
\left(\max \left\{0,2-x-\frac{e^{\theta}}{(x+1)^{2}}\right\}, 1\right] & H^{2} \in\{(x, Y): x \in[0, b(\theta)]\} \\
\left.2-x-\frac{e^{\theta}}{(x+1)^{2}}\right\} & H^{2} \in\left\{(x, Y): x \in\left[b(\theta), \sqrt{e^{\theta}}-1\right]\right\} \\
(1-x, 1] & H^{2} \in\left\{(x, Y): x \in\left[\sqrt{e^{\theta}}-1,1\right]\right\} \\
{[0,1]} & H^{2} \in\{(x, N): x \in[0,1]\} .
\end{array}\right.
$$

3. $\theta \in\left[\ln \frac{9}{4}, \simeq \ln 2.37\right]$

$$
S B R^{\beta}\left(H^{2}\right) \in\left\{\begin{array}{cc}
\left(\max \left\{0,2-x-\frac{e^{\theta}}{(x+1)^{2}}\right\}, 1\right] & \text { if } H^{2} \in\{(x, Y): x \in[0, b(\theta)]\} \\
\left\{2-x-\frac{e^{\theta}}{(x+1)^{2}}\right\} & \text { if } H^{2} \in\{(x, Y): x \in[b(\theta), c(\theta)]\} \\
\left(\min \left\{2-x-\frac{e^{\theta}}{(x+1)^{2}}, 1-x\right\}, 1\right] & \text { if } H^{2} \in\{(x, Y): x \in[c(\theta), 1]\} \\
{[0,1]} & \text { if } H^{2} \in\{(x, N): x \in[0,1]\} .
\end{array}\right.
$$

4. $\theta \in[\simeq \ln 2.37, \ln 4]$

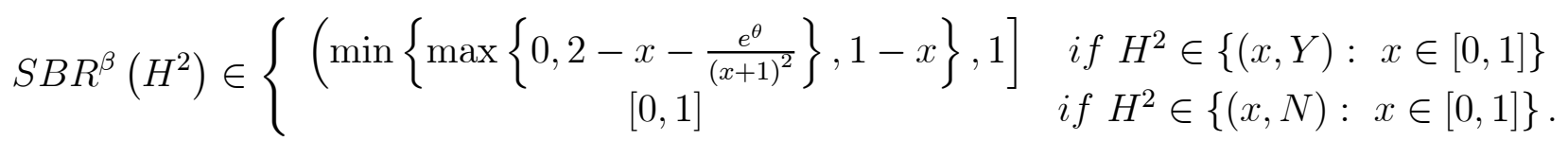

Step $2 \beta$ chooses whether to support $\alpha$ proposal on $x$, for any $x \in[0,1]$, anticipating $S B R^{\beta}\left(H^{2}\right)$ and $S B R^{\alpha}\left(H^{3}\right)$. In particular, suppose

$$
S B R^{\alpha}(\underbrace{x, S B R^{\beta}(x), S B R^{\beta}(\underbrace{x, S B R^{\beta}(x)}_{H^{2}})}_{H^{3}})=Y,
$$

then $\operatorname{SBR}^{\beta}(x)=Y$ if and only if

$$
\begin{gathered}
U^{\beta}\left(1-x-S B R^{\beta}\left(H^{2}\right), S B R^{\beta}\left(H^{2}\right) ; Y, S B R^{\alpha}\left(H^{3}\right)=Y \mid x\right) \geq \\
\geq U^{\beta}\left(1-x-S B R^{\beta}\left(H^{2}\right), S B R^{\beta}\left(H^{2}\right) ; N, S B R^{\alpha}\left(H^{3}\right)=Y \mid x\right)=U^{D} \Leftrightarrow \\
\Leftrightarrow\left(2-x-S B R^{\beta}\left(H^{2}\right)\right) \geq \frac{e^{\theta}}{\left(1+S B R^{\beta}\left(H^{2}\right)\right)^{2}} \Leftrightarrow \\
\Leftrightarrow x \leq 2-S B R^{\beta}\left(H^{2}\right)-\frac{e^{\theta}}{\left(1+S B R^{\beta}\left(H^{2}\right)\right)^{2}} .
\end{gathered}
$$

Hence 
1. when $\theta \in[0, \ln 2]$, then $\operatorname{SBR}^{\beta}(x)=Y$ if and only if

$$
\begin{gathered}
x \leq 2-S B R^{\beta}\left(H^{2}\right)-\frac{e^{\theta}}{\left(1+S B R^{\beta}\left(H^{2}\right)\right)^{2}} \Leftrightarrow \\
\Leftrightarrow\left\{\begin{array}{cc}
x \leq 2-\left(2-x-\frac{e^{\theta}}{(x+1)^{2}}\right)-\frac{e^{\theta}}{\left(\left[1+\left(2-x-\frac{e^{\theta}}{(x+1)^{2}}\right)\right]\right)^{2}} & x \in\left[0, \sqrt{e^{\theta}}-1\right] \\
x \leq 2-(1-x)-\frac{e^{\theta}}{([1+(1-x)])^{2}} & x \in\left[\sqrt{e^{\theta}}-1,2-\sqrt{e^{\theta}}\right]
\end{array} \Leftrightarrow\right. \\
\Leftrightarrow\left\{\begin{array}{cc}
(x+1)^{3} \leq(3-x)(x+1)^{2}-e^{\theta} & \text { if } x \in\left[0, \sqrt{e^{\theta}}-1\right] \\
x \leq 2-\sqrt{e^{\theta}} & \text { if } x \in\left[\sqrt{e^{\theta}}-1,2-\sqrt{e^{\theta}}\right] .
\end{array}\right.
\end{gathered}
$$

The inequality $(x+1)^{3} \leq(3-x)(x+1)^{2}-e^{\theta}$ can't be solved for a closed form, however the graphical analysis shows that it is always satisfied when $x \in\left[0, \sqrt{e^{\theta}}-1\right]$. Therefore

$$
\operatorname{SBR}^{\beta}(x) \in \begin{cases}\{Y\} & \text { if } x \in\left[0, \sqrt{e^{\theta}}-1\right] \\ \{Y\} & \text { if } x \in\left[\sqrt{e^{\theta}}-1,2-\sqrt{e^{\theta}}\right] \\ \{N\} & \text { if } x \in\left[2-\sqrt{e^{\theta}}, 1\right] .\end{cases}
$$

2. when $\theta \in\left[\ln 2, \ln \frac{9}{4}\right]$, then $S B R^{\beta}(x)=Y$ if and only if

$$
\begin{gathered}
x \leq 2-S B R^{\beta}\left(H^{2}\right)-\frac{e^{\theta}}{\left(1+S B R^{\beta}\left(H^{2}\right)\right)^{2}} \Leftrightarrow \\
\Leftrightarrow x \leq 2-\left(2-x-\frac{e^{\theta}}{(x+1)^{2}}\right)-\frac{e^{\theta}}{\left(\left[1+\left(2-x-\frac{e^{\theta}}{(x+1)^{2}}\right)\right]\right)^{2}} \quad \text { if } x \in\left[b(\theta), \sqrt{e^{\theta}}-1\right] \Leftrightarrow \\
\Leftrightarrow(x+1)^{3} \leq(3-x)(x+1)^{2}-e^{\theta} \quad \text { if } x \in\left[b(\theta), \sqrt{e^{\theta}}-1\right]
\end{gathered}
$$

The inequality $(x+1)^{3} \leq(3-x)(x+1)^{2}-e^{\theta}$ can't be solved for a closed form, however the graphical analysis shows that it is always satisfied when $x \in\left[b(\theta), \sqrt{e^{\theta}}-1\right]$. Therefore

$$
S B R^{\beta}(x) \in \begin{cases}\{Y\} & \text { if } x \in\left[b(\theta), \sqrt{e^{\theta}}-1\right] \\ \{N\} & \text { if } x \in[0, b(\theta)] \cup\left[\sqrt{e^{\theta}}-1,1\right] .\end{cases}
$$


3. when $\theta \in\left[\ln \frac{9}{4}, \simeq \ln 2.37\right]$, then $S B R^{\beta}(x)=Y$ if and only if

$$
\begin{gathered}
x \leq 2-S B R^{\beta}\left(H^{2}\right)-\frac{e^{\theta}}{\left(1+S B R^{\beta}\left(H^{2}\right)\right)^{2}} \Leftrightarrow \\
\Leftrightarrow x \leq 2-\left(2-x-\frac{e^{\theta}}{(x+1)^{2}}\right)-\frac{e^{\theta}}{\left(\left[1+\left(2-x-\frac{e^{\theta}}{(x+1)^{2}}\right)\right]\right)^{2}} \quad \text { if } x \in[b(\theta), c(\theta)] \Leftrightarrow \\
\Leftrightarrow(x+1)^{3} \leq(3-x)(x+1)^{2}-e^{\theta} \quad \text { if } x \in[b(\theta), c(\theta)]
\end{gathered}
$$

The inequality $(x+1)^{3} \leq(3-x)(x+1)^{2}-e^{\theta}$ can't be solved for a closed form, however the graphical analysis shows that it is always satisfied when $x \in[b(\theta), c(\theta)]$. Therefore

$$
\operatorname{SBR}^{\beta}(x) \in \begin{cases}\{Y\} & \text { if } x \in[b(\theta), c(\theta)] \\ \{N\} & \text { if } x \in[0, b(\theta)] \cup[c(\theta), 1] .\end{cases}
$$

4. when $\theta \in[\simeq \ln 2.37, \ln 4]$, then

$$
\operatorname{SBR}^{\beta}(x) \in\{N\} \forall x \in[0,1]
$$

because if $S B R^{\beta}(x)=Y$ then $S B R^{\beta}\left(H^{2}\right) \in\left(\min \left\{\max \left\{0,2-x-\frac{e^{\theta}}{(x+1)^{2}}\right\}, 1-x\right\}, 1\right]$ and then $S B R^{\alpha}\left(H^{3}\right)=N$, if $S B R^{\beta}(x)=N$ then $S B R^{\beta}\left(H^{2}\right) \in[0,1]$ and then $S B R^{\alpha}\left(H^{3}\right) \in\{Y, N\}$, however in both case

$$
U^{\beta}\left(1-x-S B R^{\beta}\left(H^{2}\right), S B R^{\beta}\left(H^{2}\right) ; Y, S B R^{\alpha}\left(H^{3}\right) \mid x\right)=U^{D} .
$$

Then, we might sum $S B R^{\beta}(x)$ as follows:

1. when $\theta \in[0, \ln 2]$, then

$$
\operatorname{SBR}^{\beta}(x) \in \begin{cases}\{Y\} & \text { if } x \in\left[0,2-\sqrt{e^{\theta}}\right] \\ \{N\} & \text { if } x \in\left[2-\sqrt{e^{\theta}}, 1\right] .\end{cases}
$$

2. when $\theta \in\left[\ln 2, \ln \frac{9}{4}\right]$, then

$$
\operatorname{SBR}^{\beta}(x) \in \begin{cases}\{N\} & \text { if } x \in[0, b(\theta)] \\ \{Y\} & \text { if } x \in\left[b(\theta), \sqrt{e^{\theta}}-1\right] \\ \{N\} & \text { if } x \in\left[\sqrt{e^{\theta}}-1,1\right]\end{cases}
$$


3. when $\theta \in\left[\ln \frac{9}{4}, \simeq \ln 2.37\right]$, then

$$
\operatorname{SBR}^{\beta}(x)=\left\{\begin{array}{cc}
N & \text { if } x \in[0, b(\theta)] \\
Y & \text { if } x \in[b(\theta), c(\theta)] \\
N & \text { if } x \in[c(\theta), 1] .
\end{array}\right.
$$

4. when $\theta \in[\simeq \ln 2.37, \ln 4]$, then

$$
S B R^{\beta}(x) \in\{Y, N\} \forall x \in[0,1]
$$

Step $1 \alpha$ chooses $x \in[0,1]$ to maximize

$$
U^{\alpha}\left(1-x-S B R^{\beta}\left(H^{2}\right), x ; S B R^{\beta}(x), S B R^{\alpha}\left(H^{3}\right)\right)
$$

that we will denote by $U^{\alpha}(x \mid \theta)$ to simplify. Again, suppose

$$
S B R^{\alpha}(\underbrace{x, S B R^{\beta}(x), S B R^{\beta}(\underbrace{x, S B R^{\beta}(x)}_{H^{2}})}_{H^{3}})=Y,
$$

then

1. when $\theta \in[0, \ln 2]$

$$
\begin{aligned}
& U^{\alpha}(x \mid \theta)=\left\{\begin{array}{cc}
\ln [1+1-x-\underbrace{\left(2-x-\frac{e^{\theta}}{(x+1)^{2}}\right)}_{S B R^{\beta}\left(H^{2}\right)}]+2 \ln (1+x) & x \in\left[0, \sqrt{e^{\theta}}-1\right] \\
\ln [1-x-\underbrace{(1-x)}_{S B R^{\beta}\left(H^{2}\right)}]+2 \ln (1+x) & x \in\left[\sqrt{e^{\theta}}-1,2-\sqrt{\theta}\right. \\
\theta & x \in\left[2-\sqrt{e^{\theta}}, 1\right]
\end{array}\right. \\
& =\left\{\begin{array}{cc}
\theta-2 \ln (1+x)+2 \ln (1+x)=\theta & \text { if } x \in\left[0, \sqrt{e^{\theta}}-1\right] \\
2 \ln (1+x) & \text { if } x \in\left[\sqrt{e^{\theta}}-1,2-\sqrt{e^{\theta}}\right] \\
\theta & \text { if } x \in\left[2-\sqrt{e^{\theta}}, 1\right] .
\end{array}\right.
\end{aligned}
$$


Hence

$$
\max _{x \in[0,1]} U^{\alpha}(x \mid \theta)=\left\{\begin{array}{cc}
2 \ln \left(3-\sqrt{e^{\theta}}\right) & \text { if } x=2-\sqrt{e^{\theta}} \\
\theta & \text { if } x \in[0, \sqrt{\theta}-1] \cup\left[2-\sqrt{e^{\theta}}, 1\right]
\end{array}\right.
$$

and, since

$2 \ln \left(3-\sqrt{e^{\theta}}\right) \geq \theta \Leftrightarrow\left(3-\sqrt{e^{\theta}}\right)^{2} \geq \theta \Leftrightarrow 3-\sqrt{e^{\theta}} \geq \sqrt{\theta} \Leftrightarrow \sqrt{\theta}+\sqrt{e^{\theta}} \leq 3$

which is clearly satisfied when $\theta \in[0, \ln 2]$. Then

$$
\theta \in[0, \ln 2] \Rightarrow \underset{x \in[0,1]}{\arg \max } U^{\alpha}(x \mid \theta)=2-\sqrt{e^{\theta}} .
$$

Thus

$$
\theta \in[0, \ln 2] \Rightarrow x \in\left\{2-\sqrt{e^{\theta}}\right\} \Rightarrow S B R^{\beta}(x) \in\{Y\}
$$

which in turns implies

$S B R^{\beta}\left(2-\sqrt{e^{\theta}}, Y\right) \in\{1-x\}=\left\{\sqrt{e^{\theta}}-1\right\}$ and $S B R^{\alpha}\left(2-\sqrt{e^{\theta}}, Y, \sqrt{e^{\theta}}-1\right) \in\{Y\}$.

Thus when $\theta \in[0, \ln 2]$ the subgame perfect equilibrium outcome is

$$
\left(h^{\alpha L}, x^{\alpha L}, y^{\alpha L}\right)=\left(0,2-\sqrt{e^{\theta}}, \sqrt{e^{\theta}}-1\right) .
$$

2. when $\theta \in\left[\ln 2, \ln \frac{9}{4}\right]$, then

$$
\begin{gathered}
U^{\alpha}(x \mid \theta)=\left\{\begin{array}{cc}
\theta & x \in[0, b(\theta)] \\
\ln [1+1-x-\underbrace{\left(2-x-\frac{e^{\theta}}{(x+1)^{2}}\right)}_{S B R^{\beta}\left(H^{2}\right)} & \\
\theta & x \in\left[\sqrt{e^{\theta}}-1,1\right]
\end{array}\right] \\
=\left\{\begin{array}{cr}
\theta & x \in\left[b(\theta), \sqrt{e^{\theta}}-1\right] \\
\theta-2 \ln (1+x)+2 \ln (1+x)=\theta & \text { if } x \in\left[b(\theta), \sqrt{e^{\theta}}-1\right] \\
\theta & \text { if } x \in\left[\sqrt{e^{\theta}}-1,1\right]
\end{array}\right.
\end{gathered}
$$

Hence

$$
\max _{x \in[0,1]} U^{\alpha}(x \mid \theta)=\theta
$$


Then

$$
\theta \in\left[\ln 2, \ln \frac{9}{4}\right] \Rightarrow \underset{x \in[0,1]}{\arg \max } U^{\alpha}(x \mid \theta)=[0,1] .
$$

Thus the equilibrium outcome is

$\theta \in\left[\ln 2, \ln \frac{9}{4}\right] \Rightarrow x \in[0,1] \Rightarrow S B R^{\beta}(x) \in \begin{cases}\{N\} & \text { if } x \in[0, b(\theta)] \\ \{Y\} & \text { if } x \in\left[b(\theta), \sqrt{e^{\theta}}-1\right] \\ \{N\} & \text { if } x \in\left[\sqrt{e^{\theta}}-1,1\right]\end{cases}$

which in turns implies

$\operatorname{SBR}^{\beta}(x, \cdot) \in\left\{\begin{array}{cc}{[0,1]} & \text { if }(x, N): x \in[0, b(\theta)] \cup\left[\sqrt{e^{\theta}}-1,1\right] \\ \left\{2-x-\frac{e^{\theta}}{(x+1)^{2}}\right\} & \text { if }(x, Y): x \in\left[b(\theta), \sqrt{e^{\theta}}-1\right]\end{array}\right.$

$S B R^{\alpha}\left(H^{3}\right)(x, \cdot, y) \in\left\{\begin{array}{cc}\{Y, N\} & (x, N, y):(x, y) \in[0, b(\theta)] \cup\left[\sqrt{e^{\theta}}-1,1\right] \times[0,1] \\ \{Y\} & (x, Y, y):(x, y) \in\left[b(\theta), \sqrt{e^{\theta}}-1\right] \times\left\{2-x-\frac{e^{\theta}}{(x+1)^{2}}\right\}\end{array}\right.$

Note that in this case we have a continuum of payoff equivalent subgame equilibria. To simplify and for robustness reasons, we consider the default equilibrium outcome only.

3. when $\theta \in\left[\ln \frac{9}{4}, \simeq \ln 2.37\right]$, then

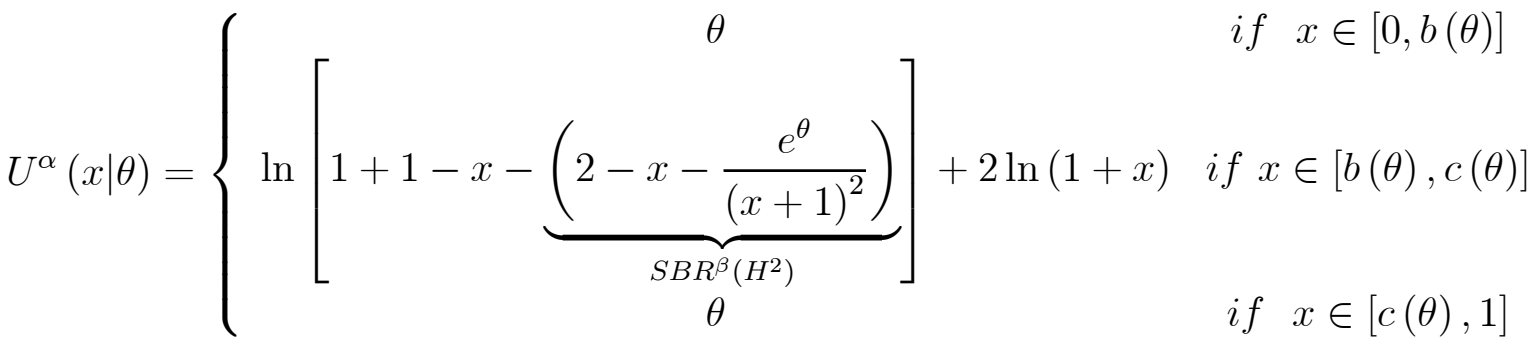

$$
\begin{aligned}
& =\left\{\begin{array}{cc}
\theta & \text { if } x \in[0, b(\theta)] \\
\theta-2 \ln (1+x)+2 \ln (1+x)=\theta & \text { if } x \in[b(\theta), c(\theta)] \\
\theta & \text { if } x \in[c(\theta), 1]
\end{array}\right.
\end{aligned}
$$

Hence

$$
\max _{x \in[0,1]} U^{\alpha}(x \mid \theta)=\theta
$$

Then

$$
\theta \in\left[\ln \frac{9}{4}, \simeq \ln 2.37\right] \Rightarrow \underset{x \in[0,1]}{\arg \max } U^{\alpha}(x \mid \theta)=[0,1]
$$


Thus the equilibrium outcome is

$\theta \in\left[\ln \frac{9}{4}, \simeq \ln 2.37\right] \Rightarrow x \in[0,1] \Rightarrow S B R^{\beta}(x) \in\left\{\begin{array}{l}\{N\} \text { if } x \in[0, b(\theta)] \\ \{Y\} \text { if } x \in[b(\theta), c(\theta)] \\ \{N\} \text { if } x \in[c(\theta), 1]\end{array}\right.$

which in turns implies

$$
\begin{aligned}
& S B R^{\beta}(x, \cdot) \in\left\{\begin{array}{cc}
{[0,1]} & \text { if }(x, N): x \in[0, b(\theta)] \cup[c(\theta), 1] \\
\left\{2-x-\frac{e^{\theta}}{(x+1)^{2}}\right\} & \text { if }(x, Y): x \in[b(\theta), c(\theta)]
\end{array}\right. \\
& S B R^{\alpha}\left(H^{3}\right)(x, \cdot, y) \in\left\{\begin{array}{c}
\{Y, N\} \quad \text { if }(x, N, y):(x, y) \in[0, b(\theta)] \cup[c(\theta), 1] \times[0,1] \\
\{Y\} \quad \text { if }(x, Y, y):(x, y) \in[b(\theta), c(\theta)] \times\left\{2-x-\frac{e^{\theta}}{(x+1)^{2}}\right\}
\end{array}\right.
\end{aligned}
$$

Again, also in this case we have a continuum of payoff equivalent subgame equilibria and we consider the default equilibrium outcome only.

4. when $\theta \in[\simeq \ln 2.37, \ln 4]$, then

$$
U^{\alpha}(x \mid \theta)= \begin{cases}\theta & \text { if } x \in[0,1]\end{cases}
$$

Hence

$$
\max _{x \in[0,1]} U^{\alpha}(x \mid \theta)= \begin{cases}\theta & \text { if } \quad x \in[0,1]\end{cases}
$$

then

$$
\theta \in[\simeq \ln 2.37, \ln 4] \Rightarrow \underset{x \in[0,1]}{\arg \max } U^{\alpha}(x \mid \theta)=[0,1] .
$$

In, this case either $\alpha$ or $\beta$ would refuse the logroll and the unique subgame perfect equilibrium outcome is

$$
\left(h^{\alpha L}, x^{\alpha L}, y^{\alpha L}\right)=\left(h^{D}, x^{D}, y^{D}\right) .
$$

To conclude, the subgame perfect equilibrium outcomes of the logrolling game are

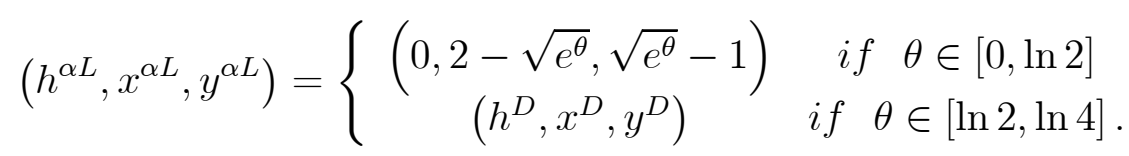

\subsection{The Efficient Benchmark}

Suppose the allocation decision is made by a utilitarian social planner whose goal is to maximize the social welfare $W$, which is the sum of groups' payoffs. Then the efficient allocation $(h, x, y)$ is derived by the following problem:

$$
\max _{(h, x, y)} W(h, x, y)=U^{\alpha}(h, x)+U^{\beta}(h, y)
$$




$$
\text { s.t. } x+y+h \leq 1 ; x \geq 0 ; y \geq 0 ; h \geq 0 .
$$

Since both payoff functions are strictly increasing in $x, y$ and $h$, the public budget constraint is binding which implies

$$
h=1-x-y .
$$

Hence, the social welfare problem is

$$
\max _{(h, x, y)} U^{\alpha}(h, x)+U^{\beta}(h, y) \quad \text { s.t. } h+x+y=1 ; \quad h \geq 0 ; \quad x \geq 0 ; \quad y \geq 0 .
$$

The objective function is concave and the constraint is linear, therefore the KuhnTucker conditions are both necessary and sufficient. The Lagrangian is

$$
L(x, y, h)=U^{\alpha}(h, x)+U^{\beta}(h, y)+\lambda(1-x-y-h)
$$

and the Kuhn-Tucker conditions are:

$$
\begin{gathered}
\frac{\partial L}{\partial h}=\frac{\partial U^{\alpha}(h, x)}{\partial h}+\frac{\partial U^{\beta}(h, y)}{\partial h}-\lambda \leq 0, h \geq 0, h\left(\frac{\partial U^{\alpha}(h, x)}{\partial h}+\frac{\partial U^{\beta}(h, x)}{\partial h}-\lambda\right)=0 \\
\frac{\partial L}{\partial x}=\frac{\partial U^{\alpha}(h, x)}{\partial x}-\lambda \leq 0, \quad x \geq 0, \text { and } x\left(\frac{\partial U^{\alpha}(h, x)}{\partial x}-\lambda\right)=0 \\
\frac{\partial L}{\partial y}=\frac{\partial U^{\beta}(h, y)}{\partial y}-\lambda \leq 0, \quad y \geq 0, \text { and } y\left(\frac{\partial U^{\beta}(h, y)}{\partial y}-\lambda\right)=0 \\
\lambda \geq 0, \quad x+y+h-1=0, \quad \text { and } \lambda(x+y+h-1)=0 .
\end{gathered}
$$

Since $L$ is strictly increasing in $(h, x, y)$, the constrain is binding. Then

$$
\begin{aligned}
\lambda>0 & \Rightarrow \frac{\partial L}{\partial x}=\frac{\partial U^{\alpha}(h, x)}{\partial x}-\lambda=0 \text { and } \frac{\partial U^{\beta}(h, y)}{\partial y}-\lambda=0 \Rightarrow \\
& \Rightarrow \frac{\partial U^{\alpha}(h, x)}{\partial x}=\frac{\partial U^{\beta}(h, y)}{\partial y}=\lambda=0 \Rightarrow x^{E}=y^{E}
\end{aligned}
$$

and

$$
\lambda>0 \Rightarrow \frac{\partial U^{\alpha}\left(h, x^{E}\right)}{\partial h}+\frac{\partial U^{\beta}\left(h, y^{E}\right)}{\partial h}=\lambda \Rightarrow \frac{\partial U^{\alpha}\left(h,{ }^{E}\right)}{\partial h}=\frac{1}{2} \lambda .
$$

Therefore we might conclude that the efficient allocation of the public and private goods satisfies the following inequality

$$
h^{E} \geq x^{E}=y^{E} .
$$

Moreover, comparing these FOCs with the maximizations of the logrolling process it is immediate that

$$
h^{E}>h^{L}
$$

because the logrolling incentives compatibility constraints implicitly restrain the set of possible $h$ : a player internalizes the effect of $h$ on the other's payoff as far as the incentive compatibility constraint is satisfied, which by definition is strictly less than the full effect. To exemplify, let consider two simple cases. 


\subsubsection{Example 1: Quasi Linear Payoff Functions}

The utilitarian maximization is:

$$
\begin{gathered}
\max _{(h, x, y)}\left(U^{\alpha}(h, x)+U^{\beta}(h, y)\right)=\max _{(h, x, y)}(h+\sqrt{x}+\sqrt{y}) \\
\text { s.t. } x+y+h \leq 1 ; x \in[0,1] ; y \in[0,1] ; h \in[0,1] .
\end{gathered}
$$

Since the objective function is strictly increasing in $(h, x, y)$, then

$$
h=1-x-y
$$

the Lagrangian can be written as

$$
L(x, y)=h+\sqrt{x}+\sqrt{y}+\lambda(1-x-y-h)+\mu(1-x)+\nu(1-y)+\xi(1-h)
$$

and the Kuhn-Tucker conditions for this Lagrangian are:

$$
\begin{gathered}
\frac{\partial L}{\partial h}=1-\lambda-\xi \leq 0, \quad h \geq 0, \text { and } h(1-\lambda-\xi)=0 \\
\frac{\partial L}{\partial x}=\frac{1}{2 \sqrt{x}}-\lambda-\mu \leq 0, \quad x \geq 0, \text { and } x\left(\frac{1}{2 \sqrt{x}}-\lambda-\mu\right)=0 \\
\frac{\partial L}{\partial y}=\frac{1}{2 \sqrt{y}}-\lambda-\nu \leq 0, \quad x \geq 0, \text { and } y\left(\frac{1}{2 \sqrt{y}}-\lambda-\nu\right)=0 \\
\lambda, \xi, \mu, \nu \geq 0, \quad x+y+h-1 \leq 0, h \leq 1, x \leq 1, y \leq 1, \\
\lambda(x+y+h-1)=\mu(1-x)=\nu(1-y)=\xi(1-h)=0 .
\end{gathered}
$$

Then

$$
\left\{\begin{array}{c}
\lambda=1 \\
\frac{1}{2 \sqrt{x}}=\lambda \\
\frac{1}{2 \sqrt{y}}=\lambda \\
x+y+h-1=0
\end{array}=\left\{\begin{array}{c}
\lambda=1 \\
\sqrt{x}=\frac{1}{2} \\
\sqrt{x}=\frac{1}{2} \\
h=1-x-y
\end{array}=\left\{\begin{array}{c}
\lambda=1 \\
x=\frac{1}{4} \\
x=\frac{1}{4} \\
h=\frac{1}{2} .
\end{array}\right.\right.\right.
$$

and the efficient utilitarian solution is

$$
\left(h^{E}, x^{E}, y^{E}\right)=\left(\frac{1}{2}, \frac{1}{4}, \frac{1}{4}\right) .
$$




\subsubsection{Example 2: Stone Geary Payoff Functions}

The utilitarian maximization is:

$$
\begin{gathered}
\max _{(h, x, y)}\left(U^{\alpha}(h, x)+U^{\beta}(h, y)\right)=\max _{(h, x, y)}(2 \ln (1+h)+2 \ln (1+x)+2 \ln (1+y)) \\
\text { s.t. } x+y+h \leq 1 ; x \in[0,1] ; y \in[0,1] ; h \in[0,1] .
\end{gathered}
$$

Since the objective function is strictly increasing in $(h, x, y)$, then

$$
h=1-x-y
$$

the Lagrangian can be written as

$L(x, y)=2 \ln (1+h)+2 \ln (1+x)+2 \ln (1+y)+\lambda(1-x-y-h)+\mu(1-x)+\nu(1-y)+\xi(1-h)$

and the Kuhn-Tucker conditions for this Lagrangian are:

$$
\begin{gathered}
\frac{\partial L}{\partial h}=\frac{2}{1+h}-\lambda-\xi \leq 0, \quad h \geq 0, \text { and } h\left(\frac{2}{1+h}-\lambda-\xi\right)=0 \\
\frac{\partial L}{\partial x}=\frac{2}{1+x}-\lambda-\mu \leq 0, \quad x \geq 0, \text { and } x\left(\frac{2}{1+x}-\lambda-\mu\right)=0 \\
\frac{\partial L}{\partial y}=\frac{2}{1+y}-\lambda-\nu \leq 0, \quad x \geq 0, \text { and } y\left(\frac{2}{1+y}-\lambda-\nu\right)=0 \\
\lambda, \xi, \mu, \nu \geq 0, \quad x+y+h-1 \leq 0, h \leq 1, x \leq 1, y \leq 1, \\
\lambda(x+y+h-1)=\mu(1-x)=\nu(1-y)=\xi(1-h)=0 .
\end{gathered}
$$

Then

$$
\left\{\begin{array}{c}
\frac{2}{1+h}=\lambda \\
\frac{2}{1+x}=\lambda \\
\frac{2}{1+y}=\lambda \\
x+y+h-1=0
\end{array}=\left\{\begin{array}{c}
h=x \\
x=y \\
y=x \\
x=1-x-x
\end{array}=\left\{\begin{array}{c}
\lambda=\frac{3}{2} \\
x=\frac{1}{3} \\
y=\frac{1}{3} \\
h=\frac{1}{3}
\end{array}\right.\right.\right.
$$

and the efficient utilitarian solution is

$$
\left(h^{E}, x^{E}, y^{E}\right)=\left(\frac{1}{3}, \frac{1}{3}, \frac{1}{3}\right)
$$




\subsection{An Interest Group as Dictator}

If group $\alpha$ has the decision making power, then

$$
\begin{gathered}
\max _{(h, x, y)} U^{\alpha}(h, x) \\
\text { s.t. } h+x+y \leq 1 ; h \geq 0, y \geq 0, x \geq 0 .
\end{gathered}
$$

As $\alpha$ 's objective function does not depend on $y$, then

$$
y^{\alpha}=0
$$

so that

$$
h=1-x .
$$

The objective function is concave and the constraint is linear, therefore the KuhnTucker conditions are necessary and sufficient:

$$
\begin{gathered}
\frac{d L}{d x}=-\frac{\partial U^{\alpha}(1-x, x)}{\partial h}+\frac{\partial U^{\alpha}(1-x, x)}{\partial x}-\lambda \leq 0, x \geq 0, \\
x\left(-\frac{\partial U^{\alpha}(1-x, x)}{\partial h}+\frac{\partial U^{\alpha}(1-x, x)}{\partial x}-\lambda\right)=0 \\
\lambda \geq 0, \quad x-1 \leq 0, \text { and } \lambda(x-1)=0
\end{gathered}
$$

Since $L$ is strictly increasing in $x$ then

$$
\begin{gathered}
\frac{d L}{d x}=-\frac{\partial U^{\alpha}(1-x, x)}{\partial h}+\frac{\partial U^{\alpha}(1-x, x)}{\partial x}-\lambda=0 \\
\lambda \geq 0, \quad x-1 \leq 0, \text { and } \lambda(x-1)=0 .
\end{gathered}
$$

Then

$$
\frac{\partial U^{\alpha}(1-x, x)}{\partial x}>\frac{\partial U^{\alpha}(1-x, x)}{\partial h} \Rightarrow \lambda>0 \Rightarrow x^{\alpha}=1 \text { and } h^{\alpha}=0 .
$$

Hence

$$
x^{\alpha}=1>x^{E} ; \quad h^{\alpha}=0<h^{E} ; y^{\alpha}=0<y^{E} .
$$

\subsubsection{Example 1: Quasi Linear Payoff Functions}

Group $\alpha$ will maximize its group welfare

$$
\begin{aligned}
& \max _{(h, x, y)} U^{\alpha}=\max _{(h, x, y)}\left(\frac{1}{2} h+\sqrt{x}\right) \\
& \text { s.t. } h+x+y \leq 1 ; h \geq 0, y \geq 0, x \geq 0 .
\end{aligned}
$$


Since the objective function doesn't depend on $y$,

$$
y^{\alpha}=0
$$

and

$$
h=1-x .
$$

Hence the Lagrangian can be written as

$$
L(x, y)=\frac{1}{2} h+\sqrt{x}+\lambda(1-x-h)
$$

and the Kuhn-Tucker conditions for this Lagrangian are:

$$
\begin{gathered}
\frac{\partial L}{\partial h}=\frac{1}{2}-\lambda \leq 0, \quad h \geq 0, \text { and } h\left(\frac{1}{2}-\lambda\right)=0 \\
\frac{\partial L}{\partial x}=\frac{1}{2 \sqrt{x}}-\lambda \leq 0, \quad x \geq 0, \text { and } x\left(\frac{1}{2 \sqrt{x}}-\lambda\right)=0 \\
\lambda \geq 0, \quad x+h-1 \leq 0, h \in[0,1], x \in[0,1], \lambda(x+h-1)=0 .
\end{gathered}
$$

Then

$$
\left\{\begin{array}{c}
\lambda=\frac{1}{2} \\
\frac{1}{2 \sqrt{x}}=\lambda \\
x+h-1=0
\end{array}=\left\{\begin{array}{c}
\lambda=\frac{1}{2} \\
\frac{1}{2 \sqrt{x}}=\frac{1}{2} \\
h=1-x
\end{array}=\left\{\begin{array}{c}
\lambda=\frac{1}{2} \\
x=1 \\
h=0 .
\end{array}\right.\right.\right.
$$

Thus the solution of the problem is

$$
\left(h^{\alpha}, x^{\alpha}, y^{\alpha}\right)=(0,1,0) .
$$

\subsubsection{Example 2: Stone Geary Payoff Functions}

Group $\alpha$ will maximize its group welfare

$$
\begin{gathered}
\max _{(h, x, y)} U^{\alpha}=\max _{(h, x, y)}(\ln (1+h)+2 \ln (1+x)) \\
\text { s.t. } h+x+y \leq 1 ; h \geq 0, y \geq 0, x \geq 0 .
\end{gathered}
$$

Since the objective function doesn't depend on $y$,

$$
y^{\alpha}=0
$$

and

$$
h=1-x .
$$

Hence the Lagrangian can be written as

$$
L(x, y)=\ln (1+h)+2 \ln (1+x)+\lambda(1-x-h)
$$


and the Kuhn-Tucker conditions for this Lagrangian are:

$$
\begin{gathered}
\frac{\partial L}{\partial h}=\frac{1}{1+h}-\lambda \leq 0, \quad h \geq 0, \text { and } h\left(\frac{1}{1+h}-\lambda\right)=0 \\
\frac{\partial L}{\partial x}=\frac{2}{1+x}-\lambda \leq 0, \quad x \geq 0, \text { and } x\left(\frac{2}{1+x}-\lambda\right)=0 \\
\lambda \geq 0, \quad x+h-1 \leq 0, h \in[0,1], x \in[0,1], \lambda(x+h-1)=0 .
\end{gathered}
$$

Then

$$
\left\{\begin{array}{c}
\frac{1}{1+h}=\lambda \\
\frac{2}{1+x}=\lambda \\
x+h-1=0
\end{array}=\left\{\begin{array}{c}
\frac{1}{1+h}=\frac{2}{1+x} \\
\frac{2}{1+x}=\lambda \\
h=1-x
\end{array}=\left\{\begin{array}{c}
1+x=2(1+1-x) \\
\frac{2}{1+x}=\lambda \\
h=1-x
\end{array}=\left\{\begin{array}{l}
x=1 \\
1=\lambda \\
h=0
\end{array}\right.\right.\right.\right.
$$

Thus the solution of the problem is

$$
\left(h^{\alpha}, x^{\alpha}, y^{\alpha}\right)=(0,1,0) .
$$

\subsection{Legislative Bargaining}

Consider the following legislative bargaining procedure:

1. one of the interest groups, say $\alpha$, makes a policy proposal $(h, x, y)$;

2. the other group $\beta$ chooses whether to accept or to reject the proposal;

3. if accepted, the proposal is implemented; if not, a default outcome $\left(h^{D}, x^{D}, y^{D}\right)$ gets implemented, such that both players will get a reservation utility

$$
U^{D}=\theta<U^{\alpha}(0,1,0)=U^{\beta}(0,0,1) .
$$

$>$ From a formal point of view, this is equivalent to an ultimatum game, where the object of interaction is a bundle of public and private goods $(h, x, y)$.

We can find the subgame perfect equilibrium of this simple game by backward induction.

Step 2 Group $\beta$ chooses between to accept the policy proposed by $\alpha$ and the default policy. $\beta$ will reject the proposal by the agenda setter $\alpha$ if it is not getting at least as high a payoff from the policy proposal as from the default policy: ${ }^{19} \beta$ will accept the proposal $\left(h^{\alpha}, x^{\alpha}, y^{\alpha}\right)$ if and only if

$$
S B R^{\beta}\left(h^{\alpha}, x^{\alpha}, y^{\alpha}\right)=Y \Leftrightarrow U^{\beta}\left(h^{\alpha}, y^{\alpha}\right) \geq U^{D}=\theta \Leftrightarrow y^{\alpha} \in B^{\alpha}\left(h^{\alpha}, \theta\right)
$$

\footnotetext{
${ }^{19}$ As usual it is assumed that a group will accept a proposal when indifferent between it and the alternative.
} 
where by construction $y^{\alpha} \in B^{\alpha}\left(h^{\alpha}, \theta\right)$ is the solution of the inequality IC $\mathrm{LB}$, that - restricted to $[0,1]$ - is called $\beta$ 's "incentive compatibility legislative bargaining constraint". Then, we can write the following sequential best reply for $\alpha$ :

$$
\operatorname{SBR}^{\beta}\left(h^{\alpha}, x^{\alpha}, y^{\alpha}\right) \in\left\{\begin{array}{lll}
\{Y\} & \text { if } & y^{\alpha} \in B^{\alpha}\left(h^{\alpha}, \theta\right) \cap[0,1] \\
\{N\} & \text { if } & y^{\alpha} \notin B^{\alpha}\left(h^{\alpha}, \theta\right) \cap[0,1]
\end{array}\right.
$$

Note that the fact that $U^{\beta}$ is increasing in both arguments implies that

$$
\begin{gathered}
h^{\alpha \prime} \geq h^{\alpha} \Rightarrow B^{\alpha}\left(h^{\alpha \prime}, \theta\right) \subseteq B^{\alpha}\left(h^{\alpha}, \theta\right) \\
\theta^{\prime}>\theta \Rightarrow B^{\alpha}\left(h^{\alpha}, \theta^{\prime}\right) \subset B^{\alpha}\left(h^{\alpha}, \theta\right)
\end{gathered}
$$

that is the properties of the incentive compatibility legislative bargaining constraint are different from the properties of the incentive compatibility logrolling constraint because in this game $h$ and $x$ are strategic substitute. Moreover, because of our assumptions $h$ is less costly but also less effective than $y$, however this strategic substitutability implies a smaller distortion in the public good provision w.r.t. the logrolling game, as our examples will show. In particular the marginal rate of substitution of $h$ and $y$ is

$$
\forall(h, y) \in[0,1] \times[0,1] \quad \frac{d y}{d h}=-\frac{\frac{\partial U^{\beta}}{\partial h}}{\frac{\partial U^{\beta}}{\partial y}} \in(-1,0)
$$

however the complication for a full general analysis is that we might have to deal with corner solutions and with the possibility that $\theta$ is such that $\beta$ prefers to reject any proposal suitable to $\alpha$, who, in turn, may similarly prefer a default outcome, i.e. a big $\theta$ make refusal choices strategic complements.

Step 1 knowing this, $\alpha$ will maximize its own utility (??) subject to the "incentive constraint" (IC LB) and to the feasibility constraint:

$\max _{(h, x, y)} U^{\alpha}(h, x) \quad$ s.t. $\quad \theta-U^{\beta}\left(h^{\alpha}, y^{\alpha}\right) \leq 0 ; \quad h+x+y-1 \leq 0 ; \quad h \geq 0 ; \quad x \geq 0 ; \quad y \geq 0$.

The objective function is concave and the constraints are convex, therefore the Kuhn-Tucker conditions are both necessary and sufficient. The Lagrangian is

$$
L(h, x, y)=U^{\alpha}(h, x)-\zeta\left[\theta-U^{\beta}(h, y)\right]-\lambda(h+x+y-1)
$$

so that

$\frac{d L}{d h}=\frac{\partial U^{\alpha}(h, x)}{\partial h}+\zeta \frac{\partial U^{\beta}(h, y)}{\partial h}-\lambda \leq 0, h \geq 0, h\left[\frac{\partial U^{\alpha}(h, x)}{\partial h}+\zeta \frac{\partial U^{\beta}(h, y)}{\partial h}-\lambda\right]=0$ 


$$
\begin{gathered}
\frac{d L}{d x}=\frac{\partial U^{\alpha}(h, x)}{\partial x}-\lambda \leq 0, x \geq 0, x\left[\frac{\partial U^{\alpha}(h, x)}{\partial x}-\lambda\right]=0 \\
\frac{d L}{d y}=\zeta \frac{\partial U^{\beta}(h, y)}{\partial y}-\lambda \leq 0, y \geq 0, y\left[\zeta \frac{\partial U^{\beta}(h, y)}{\partial y}-\lambda\right]=0
\end{gathered}
$$

Comparing these FOCs with the conditions for an efficient allocations, it is clear that the public good choice in a legislative bargaining setting depends on the value of the Lagrange multiplier of the IC bargaining constraint $\zeta$. As well known, the value of the Lagrange multiplier at the solution of the problem is equal to the rate of change in the maximal value of the objective function as the constraint is relaxed, hence $\zeta=1$ if and only if group $\alpha$ aims fully reflect group $\beta$ aims because of the "incentive constraint", which in turn depends on the default utility level. Unfortunately, we can say more about the public good allocation in this setting without specifying the groups' payoffs. However, comparing the Kuhn-Tucker conditions for the efficient allocation with this legislative bargaining case, it is immediate that

$$
h^{\alpha L B} \leq h^{E}, \quad x^{\alpha L B} \geq x^{E}, \quad y^{\alpha L B} \leq y^{E} .
$$

\subsubsection{Example 1: Quasi Linear Payoff Functions}

We solve this simple game by backward induction.

Step 2 Group $\beta$ will reject the proposal by the agenda setter $\alpha$ if it is not getting at least as high a payoff from the policy proposal as from the default policy:

$$
U^{\beta}(h, y) \geq U^{D} \Leftrightarrow \frac{1}{2} h+\sqrt{y} \geq \theta \Leftrightarrow h \geq 2(\theta-\sqrt{y}) \Leftrightarrow \begin{cases}y \geq\left(\theta-\frac{1}{2} h\right)^{2} & \text { if } h \leq 2 \theta \\ y \geq 0 & \text { if } h \geq 2 \theta .\end{cases}
$$

Step 1 Knowing this, the agenda setter $\alpha$ will maximize its own utility (??) subject to the previous "incentive constraint". Thus:

1. if $h \geq 2 \theta$, then

$$
\begin{gathered}
1 \geq h \geq 2 \theta \Rightarrow \theta \leq \frac{1}{2} . \\
\max _{(h, x . y)}\left(\frac{1}{2} h+\sqrt{x}\right) \\
\text { s.t. } h+x+y-1 \leq 0 ; x \geq 0 ; y \geq 0 ;
\end{gathered}
$$

hence

$$
y^{\alpha B}=0, \quad h=1-x \Rightarrow \max _{x}\left(\frac{1}{2}-\frac{1}{2} x+\sqrt{x}\right)
$$


The objective function is concave, therefore the FOCs are both necessary and sufficient

$$
-\frac{1}{2}+\frac{1}{2 \sqrt{x}} \geq 0 \Leftrightarrow \frac{1}{2 \sqrt{x}} \geq \frac{1}{2} \Leftrightarrow \sqrt{x} \leq 1 \Rightarrow x^{\alpha B}=1-2 \theta \Leftrightarrow h^{\alpha B}=2 \theta .
$$

Now, we should check that this is a global maximum, that is that

$$
U^{\alpha}(2 \theta, 1-2 \theta) \geq \theta \Leftrightarrow \frac{1}{2} 2 \theta+\sqrt{1-2 \theta} \geq \theta \Leftrightarrow \sqrt{1-2 \theta} \geq 0
$$

which is always satisfied;

2. if $h \leq 2 \theta$, then $\theta \in[0,1]$ and the maximization problem is

$$
\begin{gathered}
\max _{(h, x)}\left(\frac{1}{2} h+\sqrt{x}\right) \\
\text { s.t. } y \geq\left(\theta-\frac{1}{2} h\right)^{2} ; h+x+y-1 \leq 0 ; \quad h \geq 0 ; x \geq 0 ; y \geq 0 .
\end{gathered}
$$

Since the payoff is increasing in both $h, x$, then $\alpha$ will chose the smallest possible $y$ so that both constraints are binding. Hence

$$
\begin{gathered}
h=2(\theta-\sqrt{y}) \Rightarrow 2(\theta-\sqrt{y})+y+x=1 \Leftrightarrow x=1-y-2 \theta+2 \sqrt{y} \Leftrightarrow \\
\Leftrightarrow \max _{y}(\theta-\sqrt{y}+\sqrt{1-y-2 \theta+2 \sqrt{y}}) .
\end{gathered}
$$

The objective function is concave, therefore the FOCs are both necessary and sufficient

$$
\begin{gathered}
\Leftrightarrow-\frac{1}{2 \sqrt{y}}+\frac{-1+2 \frac{1}{2 \sqrt{y}}}{2 \sqrt{1-y-2 \theta+2 \sqrt{y}}} \geq 0 \Leftrightarrow \frac{-1+\frac{1}{\sqrt{y}}}{\sqrt{1-y-2 \theta+2 \sqrt{y}}} \geq \frac{1}{\sqrt{y}} \Leftrightarrow \\
\Leftrightarrow y \leq 1-2 \sqrt{1-\theta}+1-\theta=(2-\theta-2 \sqrt{1-\theta})
\end{gathered}
$$

Let us check that

$$
\begin{gathered}
(2-\theta-2 \sqrt{1-\theta}) \in[0,1] \Leftrightarrow\left\{\begin{array} { l } 
{ 2 - \theta - 2 \sqrt { 1 - \theta } \geq 0 } \\
{ 2 - \theta - 2 \sqrt { 1 - \theta } \leq 1 }
\end{array} \Leftrightarrow \left\{\begin{array}{c}
\sqrt{1-\theta} \leq 1-\frac{\theta}{2} \\
2 \sqrt{1-\theta} \geq 1-\theta
\end{array} \Leftrightarrow\right.\right. \\
\Leftrightarrow 1-\theta \leq 1-\theta+\frac{1}{4} \theta^{2} \Leftrightarrow \frac{1}{4} \theta^{2} \geq 0
\end{gathered}
$$

which is always satisfied. Therefore, the local solution is

$y=2-\theta-2 \sqrt{1-\theta} \Rightarrow h=2[\theta-(1-\sqrt{1-\theta})]=2[\sqrt{1-\theta}-(1-\theta)] \Rightarrow$ 


$$
\Rightarrow x=1-2+\theta+2 \sqrt{1-\theta}-2 \theta+2-2 \sqrt{1-\theta}=1-\theta
$$

Now, let we check that

$$
\begin{gathered}
2[\sqrt{1-\theta}-(1-\theta)] \in[0,1] \Leftrightarrow\left\{\begin{array} { c } 
{ \sqrt { 1 - \theta } - ( 1 - \theta ) \geq 0 } \\
{ 2 \sqrt { 1 - \theta } - 2 + 2 \theta \leq 1 }
\end{array} \Leftrightarrow \left\{\begin{array}{c}
\sqrt{1-\theta} \geq 1-\theta \\
2 \sqrt{1-\theta} \leq 3-2 \theta
\end{array} \Leftrightarrow \Rightarrow\right.\right. \\
\Leftrightarrow 4-4 \theta \leq 9-12 \theta+4 \theta^{2} \Leftrightarrow 4 \theta^{2}-8 \theta+5 \geq 0
\end{gathered}
$$

which is always satisfied. Now compare this local maximum with $\theta$ :

$$
\begin{gathered}
U^{\alpha}(2[\sqrt{1-\theta}-(1-\theta)], 1-\theta) \geq \theta \Leftrightarrow \sqrt{1-\theta}-(1-\theta)+\sqrt{1-\theta} \geq \theta \Leftrightarrow \\
\Leftrightarrow-1+\theta+2 \sqrt{1-\theta} \geq \theta \Leftrightarrow \\
\Leftrightarrow \sqrt{1-\theta} \geq \frac{1}{2} \Leftrightarrow 1-\theta \geq \frac{1}{4} \Leftrightarrow \theta \leq \frac{3}{4} .
\end{gathered}
$$

Finally, for a global maximum we need to compare $U^{\alpha}(2 \theta, 1-2 \theta)$ and $U^{\alpha}(2[\sqrt{1-\theta}-(1-\theta)], 1$ when $\theta \in\left[0, \frac{1}{2}\right]$ :

$$
\begin{gathered}
U^{\alpha}(2 \theta, 1-2 \theta) \geq U^{\alpha}(2[\sqrt{1-\theta}-(1-\theta)], 1-\theta) \Leftrightarrow \\
\Leftrightarrow \frac{1}{2} 2 \theta+\sqrt{1-2 \theta} \geq \sqrt{1-\theta}-(1-\theta)+\sqrt{1-\theta} \Leftrightarrow \\
\Leftrightarrow 1+\sqrt{1-2 \theta} \geq 2 \sqrt{1-\theta} \Leftrightarrow 1+2 \sqrt{1-2 \theta}+1-2 \theta \geq 4-4 \theta \Leftrightarrow \\
\Leftrightarrow \sqrt{1-2 \theta} \geq 1-\theta \Leftrightarrow 1-2 \theta \geq 1-2 \theta+\theta^{2}
\end{gathered}
$$

which is impossible.

To conclude, we get the following solutions:

$$
\begin{gathered}
\theta \in\left[0, \frac{3}{4}\right] \Rightarrow h^{\alpha L B}=2[\sqrt{1-\theta}-(1-\theta)], \quad x^{\alpha L B}=1-\theta, \quad y^{\alpha L B}=2-\theta-2 \sqrt{1-\theta} \\
\theta \in\left[\frac{3}{4}, 1\right] \Rightarrow h^{\alpha L B}=h^{D}, \quad x^{\alpha L B}=x^{D}, \quad y^{\alpha L B}=y^{D} .
\end{gathered}
$$




\subsubsection{Example 2: Stone Geary Payoff Functions}

We solve this simple game by backward induction.

Step 2 Group $\beta$ will reject the proposal by the agenda setter $\alpha$ if it is not getting at least as high a payoff from the policy proposal as from the default policy:

$$
\begin{aligned}
& U^{\beta}(h, y) \geq U^{D} \Leftrightarrow \ln (1+h)+2 \ln (1+y) \geq \theta \Leftrightarrow(1+h)(1+y)^{2} \geq e^{\theta} \Leftrightarrow \\
& \Leftrightarrow(1+y)^{2} \geq \frac{e^{\theta}}{(1+h)} \Leftrightarrow y \geq \sqrt{\frac{e^{\theta}}{(1+h)}}-1 .
\end{aligned}
$$

Let now check whether

$$
\begin{aligned}
\sqrt{\frac{e^{\theta}}{(1+h)}-1} & \in[0,1] \Leftrightarrow\left\{\begin{array} { c } 
{ \sqrt { \frac { e ^ { \theta } } { ( 1 + h ) } } - 1 \geq 0 } \\
{ \sqrt { \frac { e ^ { \theta } } { ( 1 + h ) } } - 1 \leq 1 }
\end{array} \Leftrightarrow \left\{\begin{array}{c}
\frac{e^{\theta}}{(1+h)} \geq 1 \\
\frac{e^{\theta}}{(1+h)} \leq 4
\end{array} \Leftrightarrow\right.\right. \\
& \Leftrightarrow\left\{\begin{array} { c } 
{ e ^ { \theta } \geq ( 1 + h ) } \\
{ e ^ { \theta } \leq 4 ( 1 + h ) }
\end{array} \Leftrightarrow \left\{\begin{array}{c}
h \leq e^{\theta}-1 \\
h \geq \frac{1}{4} e^{\theta}-1 .
\end{array}\right.\right.
\end{aligned}
$$

The second condition is satisfied $\forall \theta \in[0, \ln 4]$, while the first inequality is always satisfied $\forall \theta \in[\ln 2, \ln 4]$ only, restricting the set of possible $h$ when $\theta \in$ $[0, \ln 2]$. Then, $\beta$ sequential best reply at $H^{1} \in\{(h, y): h \in[0,1], y \in[0,1]\}$ is

$$
\begin{aligned}
& \theta \in[0, \ln 2] \Rightarrow S B R^{\beta}(h, y) \in \begin{cases}\{Y\} & H^{2} \in\left\{(h, y): h \in\left[0, e^{\theta}-1\right], y \in\left[\sqrt{\frac{e^{\theta}}{(+h)}}-1,1\right]\right\} \\
\{Y\} & H^{2} \in\left\{(h, y): h \in\left[e^{\theta}-1,1\right], y \in[0,1]\right\} . \\
\{N\} & H^{2} \in\left\{(h, y): h \in\left[0, e^{\theta}-1\right], y \in\left[0, \sqrt{\frac{e^{\theta}}{(1+h)}}-1\right]\right\}\end{cases} \\
& \theta \in[\ln 2, \ln 4] \Rightarrow S B R^{\beta}(h, y) \in \begin{cases}\{Y\} & \text { if } H^{2} \in\left\{(h, y): h \in[0,1], y \in\left[\sqrt{\frac{e^{\theta}}{(1+h)}}-1,1\right]\right\} \\
\{N\} & \text { if } H^{2} \in\left\{(h, y): h \in[0,1], y \in\left[0, \sqrt{\frac{e^{\theta}}{(1+h)}}-1\right]\right\}\end{cases}
\end{aligned}
$$

Step 1 Knowing this, the agenda setter $\alpha$ will maximize its own utility (??) subject to the previous "incentive constraint". Thus:

1. when $\theta \in[0, \ln 2]$ then

(a) if $h \in\left[0, e^{\theta}-1\right]$, the maximization problem is

$$
\begin{aligned}
& \max _{(h, x)}(\ln (h+1)+2 \ln (1+x))
\end{aligned}
$$

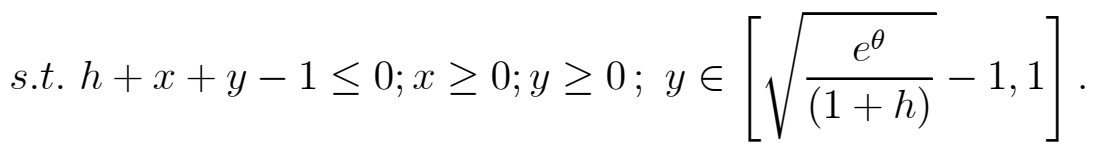


hence

$$
y^{\alpha B}=\sqrt{\frac{e^{\theta}}{(1+h)}}-1, \quad x=1-h-\left(\sqrt{\frac{e^{\theta}}{(1+h)}}-1\right) \Rightarrow x=2-h-\sqrt{\frac{e^{\theta}}{(1+h)}}
$$

and the maximization problem now is

$$
\max _{h}\left(\ln (h+1)+2 \ln \left(3-h-\sqrt{\frac{e^{\theta}}{(1+h)}}\right)\right)
$$

The objective function is concave, therefore the FOCs are both necessary and sufficient

$$
\begin{gathered}
\frac{1}{h+1}+\frac{2}{3-h-\sqrt{\frac{e^{\theta}}{(1+h)}}}\left(-1-\frac{1}{2 \sqrt{\frac{e^{\theta}}{(1+h)}}}\left(-\frac{e^{\theta}}{(1+h)^{2}}\right)\right) \geq 0 \Leftrightarrow \\
\Leftrightarrow \frac{1}{h+1}-\frac{2}{3-h-\sqrt{\frac{e^{\theta}}{(1+h)}}}+\frac{\sqrt{\frac{e^{\theta}}{(1+h)^{3}}}}{3-h-\sqrt{\frac{e^{\theta}}{(1+h)}}} \geq 0 \Leftrightarrow \\
\Leftrightarrow \frac{3-h-\sqrt{\frac{e^{\theta}}{(1+h)}}-2(h+1)+(h+1) \sqrt{\frac{e^{\theta}}{(1+h)^{3}}}}{(h+1)}\left(3-h-\sqrt{\frac{e^{\theta}}{(1+h)}}\right) \\
\Leftrightarrow 3-h-\sqrt{\frac{e^{\theta}}{(1+h)}}-2 h-2+\sqrt{\frac{e^{\theta}}{(1+h)}} \geq 0 \Leftrightarrow h \leq \frac{1}{3} .
\end{gathered}
$$

Now, we need to compare such solution with the condition $h \in$ $\left[0, e^{\theta}-1\right]$ :

$$
\frac{1}{3} \leq e^{\theta}-1 \Leftrightarrow e^{\theta} \geq \frac{4}{3} \Leftrightarrow \theta \geq \ln \left(\frac{4}{3}\right)
$$

Hence, the local solution is

$$
\begin{gathered}
\theta \in\left[0, \ln \left(\frac{4}{3}\right)\right] \Rightarrow h^{\alpha B}=\frac{1}{3}, y^{\alpha B}=\frac{1}{2} \sqrt{3 e^{\theta}}-1, \quad x^{\alpha B}=\frac{2}{3}-\frac{1}{2} \sqrt{3 e^{\theta}} \\
\theta \in\left[\ln \left(\frac{4}{3}\right), \ln 2\right] \Rightarrow h^{\alpha B}=e^{\theta}-1, y^{\alpha B}=0, \quad x^{\alpha B}=2-e^{\theta} .
\end{gathered}
$$


Now, we should check that these are global maxima, that is that

$\theta \in\left[0, \ln \left(\frac{4}{3}\right)\right] \Rightarrow U^{\alpha}\left(\frac{1}{3}, \frac{2}{3}-\frac{1}{2} \sqrt{3 e^{\theta}}\right) \geq \theta \Leftrightarrow \ln \left(\frac{4}{3}\right)+2 \ln \left(\frac{5}{3}-\frac{1}{2} \sqrt{3 e^{\theta}}\right) \geq \theta$

which is never satisfied; moreover

$\theta \in\left[\ln \left(\frac{4}{3}\right), \ln 2\right] \Rightarrow U^{\alpha}\left(e^{\theta}-1,2-e^{\theta}\right) \geq \theta \Leftrightarrow \ln \left(e^{\theta}\right)+2 \ln \left(3-e^{\theta}\right) \geq \theta$

which is always satisfied. Hence the global solution outcome is

$$
\theta \in[0, \ln 2] \Rightarrow h^{\alpha B}=e^{\theta}-1, y^{\alpha B}=0, \quad x^{\alpha B}=2-e^{\theta} .
$$

(b) if $h \in\left[e^{\theta}-1,1\right]$, the maximization problem is

$$
\begin{aligned}
& \max _{(h, x)}(\ln (h+1)+2 \ln (1+x)) \\
& \text { s.t. } h+x+y-1 \leq 0 ; x \geq 0 ; y \geq 0 ;
\end{aligned}
$$

hence

$$
y^{\alpha B}=0, \quad x=1-h
$$

and the maximization problem now is

$$
\max _{x}(\ln (h+1)+2 \ln (2-h))
$$

The objective function is concave, therefore the FOCs are both necessary and sufficient

$$
\frac{1}{h+1}-\frac{2}{2-h} \geq 0 \Leftrightarrow 2-h \geq 2 h+2 \Leftrightarrow 2-h \geq 2 h+2 \Leftrightarrow h \leq 0
$$

which is impossible. It means that we have a corner solution, i.e.

$$
h^{\alpha B}=e^{\theta}-1, y^{\alpha B}=0, \quad x^{\alpha B}=2-e^{\theta} .
$$

Now, we should check that these are global maxima, that is that

$\theta \in\left[0, \ln \left(\frac{4}{3}\right)\right] \Rightarrow U^{\alpha}\left(\frac{1}{3}, \frac{2}{3}-\frac{1}{2} \sqrt{3 e^{\theta}}\right) \geq \theta \Leftrightarrow \ln \left(\frac{4}{3}\right)+2 \ln \left(\frac{5}{3}-\frac{1}{2} \sqrt{3 e^{\theta}}\right) \geq \theta$

which is never satisfied; moreover

$$
\begin{aligned}
& \theta \in\left[\ln \left(\frac{4}{3}\right), \ln 2\right] \Rightarrow U^{\alpha}\left(e^{\theta}-1,2-e^{\theta}\right) \geq \theta \Leftrightarrow \ln \left(e^{\theta}\right)+2 \ln \left(3-e^{\theta}\right) \geq \theta \Leftrightarrow \\
& \Leftrightarrow 2 \ln \left(3-e^{\theta}\right) \geq 0 \Leftrightarrow\left(3-e^{\theta}\right)^{2} \geq 1 \Leftrightarrow\left(3-e^{\theta}\right) \geq 1 \text { or }\left(3-e^{\theta}\right) \leq-1 \Leftrightarrow e^{\theta} \leq 2
\end{aligned}
$$

which is always satisfied. Hence the global solution outcome is

$$
\theta \in[0, \ln 2] \Rightarrow h^{\alpha B}=e^{\theta}-1, y^{\alpha B}=0, \quad x^{\alpha B}=2-e^{\theta} .
$$


2. when $\theta \in[\ln 2, \ln 4]$ then $\alpha$ maximization problem is

$$
\begin{aligned}
& \max _{(h, x)}(\ln (h+1)+2 \ln (1+x)) \\
& \text { s.t. } h+x+y-1 \leq 0 ; x \geq 0 ; y \geq 0 ; y \in\left[\sqrt{\frac{e^{\theta}}{(1+h)}}-1,1\right] \text {. }
\end{aligned}
$$

hence

$y^{\alpha B}=\sqrt{\frac{e^{\theta}}{(1+h)}}-1, \quad x=1-h-\left(\sqrt{\frac{e^{\theta}}{(1+h)}}-1\right) \Rightarrow x=2-h-\sqrt{\frac{e^{\theta}}{(1+h)}}$

and the maximization problem now is

$$
\max _{h}\left(\ln (h+1)+2 \ln \left(3-h-\sqrt{\frac{e^{\theta}}{(1+h)}}\right)\right)
$$

The objective function is concave, therefore the FOCs are both necessary and sufficient

$$
\begin{gathered}
\frac{1}{h+1}+\frac{2}{3-h-\sqrt{\frac{e^{\theta}}{(1+h)}}}\left(-1-\frac{1}{2 \sqrt{\frac{e^{\theta}}{(1+h)}}}\left(-\frac{e^{\theta}}{(1+h)^{2}}\right)\right) \geq 0 \Leftrightarrow \\
\Leftrightarrow \frac{1}{h+1}-\frac{2}{3-h-\sqrt{\frac{e^{\theta}}{(1+h)}}}+\frac{\sqrt{\frac{e^{\theta}}{(1+h)^{3}}}}{3-h-\sqrt{\frac{e^{\theta}}{(1+h)}}} \geq 0 \Leftrightarrow \\
\Leftrightarrow \frac{3-h-\sqrt{\frac{e^{\theta}}{(1+h)}}-2(h+1)+(h+1) \sqrt{\frac{e^{\theta}}{(1+h)^{3}}}}{(h+1)\left(3-h-\sqrt{\frac{e^{\theta}}{(1+h)}}\right)} \geq 0 \Leftrightarrow \\
\Leftrightarrow 3-h-\sqrt{\frac{e^{\theta}}{(1+h)}}-2 h-2+\sqrt{\frac{e^{\theta}}{(1+h)}} \geq 0 \Leftrightarrow h \leq \frac{1}{3} .
\end{gathered}
$$

Hence, the local solution is

$$
h^{\alpha B}=\frac{1}{3}, y^{\alpha B}=\frac{1}{2} \sqrt{3 e^{\theta}}-1, \quad x^{\alpha B}=\frac{2}{3}-\frac{1}{2} \sqrt{3 e^{\theta}} .
$$

Now, we should check that these are global maxima, that is that $\theta \in[\ln 2, \ln 4] \Rightarrow U^{\alpha}\left(\frac{1}{3}, \frac{2}{3}-\frac{1}{2} \sqrt{3 e^{\theta}}\right) \geq \theta \Leftrightarrow \ln \left(\frac{4}{3}\right)+2 \ln \left(\frac{5}{3}-\frac{1}{2} \sqrt{3 e^{\theta}}\right) \geq \theta$

which is never satisfied. Hence the global solution outcome is

$$
\theta \in[\ln 2, \ln 4] \Rightarrow h^{\alpha B}=h^{D}, y^{\alpha B}=y^{D}, \quad x^{\alpha B}=x^{D} .
$$


To conclude, we get the following subgame perfect outcomes:

$$
\begin{gathered}
\theta \in[0, \ln 2] \Rightarrow h^{\alpha L B}=e^{\theta}-1, \quad x^{\alpha L B}=2-e^{\theta}, \quad y^{\alpha L B}=0 \\
\theta \in[\ln 2, \ln 4] \Rightarrow h^{\alpha L B}=h^{D}, \quad x^{\alpha L B}=x^{D}, \quad y^{\alpha L B}=y^{D} .
\end{gathered}
$$

\section{Variables and descriptive statistics for empir- ical testing}

\subsection{Variables and Descriptive statistics for the test of logrolling among ministries}

\subsubsection{Variables}

Itemized budget data for government health expenditure is not available. In this case, for the variable MOH support for Dibao, the amount of budget allocated from the medical assistance fund to directly reimburse health care services as a share of government health expenditure is used as the proxy to indicate the MOH's support for Dibao. The rationale to use this proxy is that the larger the amount of direct reimbursement of health services for Dibao recipients, the more subsidized health services are provided for Dibao recipients. In other words, the larger the amount of direct reimbursement of health services for Dibao recipients, the larger the input from $\mathrm{MOH}$ for dibao recipients. Fiscal input for mental healthcare is measured by the number of beds for mental healthcare as a share of total hospital beds. The medical assistance fund is supposed to be used to help the poor, and Dibao recipients are automatically qualified to claim from the medical assistance fund. MCA support for rural health insurance is measured by the amount of the medical assistance fund to cover the premium of rural health insurance for poor households. The descriptive statistics are shown in table 10.

\subsection{Variables and descriptive statistics for the test of inef- ficiency associated with Dibao}

\subsubsection{Variables}

The dependent variable is the ratio of the number of enrollees of unemployment insurance and total number of employees in the city. Figure ?? shows the number of unemployed and flexible employed who are under the Dibao program, which accounts for about $60 \%$ of beneficiaries who are under Dibao program.

Independent and control variables are listed as follows: 
Table 9: Descriptions of variables

\begin{tabular}{|c|c|}
\hline Variable name & Variable description \\
\hline $\begin{array}{l}\text { MOH support for } \\
\text { Dibao, } Y_{D, i, t}\end{array}$ & $\begin{array}{l}\text { share of medical assistance in government } \\
\text { health expenditure }(\%)\end{array}$ \\
\hline $\begin{array}{l}\text { MCA support for } \\
\text { rural health insur- } \\
\text { ance, } Y_{R, i, t}\end{array}$ & $\begin{array}{l}\text { share of subsidy for rural health insurance } \\
\text { in MCA budget expenditure }(\%)\end{array}$ \\
\hline $\begin{array}{ll}\text { Fiscal input for } \\
\text { mental } & \text { healthcare, } \\
X_{M, i, t-1} & \end{array}$ & $\begin{array}{l}\text { the number of beds for mental healthcare } \\
\text { as a share of total hospital beds }\end{array}$ \\
\hline $\begin{array}{l}\text { Rural health insur- } \\
\text { ance }\end{array}$ & $\begin{array}{l}\text { ratio of rural health insurance premium } \\
\text { and government health expenditure }\end{array}$ \\
\hline Dibao & $\begin{array}{l}\text { ratio of government expenditure on Dibao } \\
\text { and government civil affairs expenditure }\end{array}$ \\
\hline $\begin{array}{l}\text { Hospital doctors per } \\
\text { capita }\end{array}$ & $\begin{array}{l}\text { the number of hospital doctors per } 1000 \\
\text { people }\end{array}$ \\
\hline Urbanization & urbanization rate \\
\hline $\begin{array}{l}\text { Fiscal expenditure } \\
\text { per capita }\end{array}$ & Fiscal expenditure per capita (,000 RMB) \\
\hline $\begin{array}{l}\text { Disaster Relief ex- } \\
\text { pendtiure }\end{array}$ & $\begin{array}{l}\text { ratio of government expenditure on disas- } \\
\text { ter relief and government civil affairs ex- } \\
\text { penditure }\end{array}$ \\
\hline $\begin{array}{l}\text { Urban community } \\
\text { healthcare expendi- } \\
\text { ture }\end{array}$ & $\begin{array}{l}\text { ratio of government expenditure on urban } \\
\text { community healthcare expenditure and } \\
\text { government health expenditure }\end{array}$ \\
\hline Economic losses & $\begin{array}{l}\text { economic losses of natural disasters as a } \\
\text { share of fiscal revenue in a province }\end{array}$ \\
\hline
\end{tabular}


Table 10: Descriptive statistics of Dibao and unemployment insurance

\begin{tabular}{|l|c|c|c|c|c|}
\hline & count & mean & sd & min & max \\
\hline MOH support for Dibao & 155 & 3.268 & 3.323 & .0531 & 19.132 \\
\hline MCA support for rural health insurance & 155 & .556 & .323 & .0120 & 1.686 \\
\hline Fiscal input for mental healthcare (MOH) & 155 & 4.083 & 1.956 & .611 & 12.045 \\
\hline Fiscal input for mental healthcare (MCA) & 155 & 0.632 & 0.46 & .0159 & 2.568 \\
\hline Rural health insurance & 155 & 41.74 & 43.454 & 5.355 & 284.077 \\
\hline Hospital doctors per capita & 155 & 1.569 & 0.520 & 0.792 & 3.594 \\
\hline Fiscal expenditure per capita & 155 & 6.599 & 3.527 & 2.265 & 21.247 \\
\hline Dibao & 155 & .210 & .105 & .0280 & .455 \\
\hline Urbanization & 155 & .510 & .125 & .291 & .896 \\
\hline disaster relief & 155 & 0.065 & 0.097 & 0.0016 & 0.806 \\
\hline urban community & 155 & 3.157 & 1.998 & 0.366 & 15.745 \\
\hline economic losses of disaster & 155 & 0.204 & 0.561 & 0.0009 & 7.55 \\
\hline
\end{tabular}

Direct benefit of Dibao is defined as the among of cash benefit a Dibao recipient will get in a year (in 1,000 RMB)

$\mathrm{MOH}$ supported social assistance denotes the city level government health spending per resident (in 1,000 RMB), which indicates the level of medical assistance.

Fiscal expenditure of a prefecture city per resident (in 1,000 RMB). Fiscal expenditure measures the scale of local fiscal policies.

Gross product denotes the gross product in a prefecture city per resident (in 1,000 RMB), which measures the development stage of the local economy.

Average income: this variable refers to the average annual wage level in the city district of a city (in 1,000 RMB). The average wage is calculated on the basis of the wage level in state-owned, privately-owned as well as foreign-owned enterprises. This variable measures local conditions of economic development.

Informal sector size: This variable refers to the number of employees working in private-owned enterprises and the self employed as a share of the total labor force of a city district. This variable is relevant since we expect a larger size of the informal sector may imply that there are more people who are not willing to register as urban unemployed (Park and Cai 2011).

Year dummies are also included.

\subsubsection{Descriptive statistics}

Table 11 shows that there are 789 observations for 268 prefecture level cities in three years. The variable "employees" denotes the total number of employees in a city. "bps" denotes the number of enrollees for the urban basic pension scheme 
Table 11: Descriptive statistics of Dibao and unemployment insurance

\begin{tabular}{|l|c|c|c|c|c|}
\hline & count & mean & sd & min & $\max$ \\
\hline ui & 789 & 45.754 & 25.527 & 4.165 & 289.579 \\
\hline Direct Benefit of Dibao & 789 & 2.963 & 0.758 & 1.421 & 6.453 \\
\hline MOH supported social assistance & 789 & 0.483 & 0.161 & 0.155 & 2.344 \\
\hline education assistance & 789 & 1.144 & 0.449 & 0.0603 & 5.727 \\
\hline fiscal expenditure & 789 & 8.915 & 5.805 & 1.185 & 74.648 \\
\hline gross product & 789 & 63.820 & 50.240 & 8.406 & 466.996 \\
\hline average income & 789 & 42.732 & 9.256 & 19.267 & 92.357 \\
\hline informal sector size & 789 & .436 & .160 & .018 & 1.949 \\
\hline unemployment & 789 & 0.029 & 0.020 & 0.002 & 0.291 \\
\hline bps & 789 & 82.148 & 46.361 & 5.169 & 497.273 \\
\hline
\end{tabular}

(in 1,000). "education assistance" refers to government education expenditure per person. In some cities, the ratio of enrollees under unemployment insurance and employees is over 1. Similarly, in some cities, the ratio of workers in the size of the informal sector and the number of employees is larger than 1. The reason is that in some cities the size of the informal sector is very big and the number of workers may be much more than the number of workers hired by the registered companies (i.e. the variable "employees" and in some cases, the number of workers may also be higher than the number of local residents (e.g. the variable "people"), which is defined as people who have registered their residential status with the city and reside more than 6 months in that city. The variations across cities are large. In the richest city, the annual average income is more than RMB 90 thousands while the annual average income is RMB 19 thousands in the poorest city. The fiscal expenditure of the richest city reached RMB 74,600 per person and fiscal expenditure in the poorest city only reached RMB 1,185 per person.

\subsection{Variables and descriptive statistics for the test of inef- ficiency associated with mental healthcare}

\subsubsection{Variables}

The dependent variable is defined as the volume of inpatient services in two types of mental hospitals: $\mathrm{MOH}$ and MCA mental hospitals in a province.

The independent variables include the number of beds in two types of hospitals in a province.

The control variables such as income, education, manufacturing sector as well as insurance measure the demand for mental health care. People residing in a more economically developed region, in a better educated region, in a region with 
Table 12: Descriptive statistics

\begin{tabular}{|l|c|c|c|c|c|}
\hline & count & mean & sd & min & max \\
\hline cms & 149 & 56.921 & 18.307 & 0.051 & 82.503 \\
\hline urban disposable income & 149 & 19.3591 & 5.630 & 11.9298 & 40.1883 \\
\hline inpatients volume & 149 & 82.673 & 22.271 & 35.928 & 159.732 \\
\hline outpatients in hospitals & 149 & 4430.867 & 1496.305 & 2511.529 & 9583.209 \\
\hline outpatients in clinics & 149 & 2602.269 & 812.257 & 895.786 & 4687.936 \\
\hline doctors & 149 & 1.757 & 0.619 & .868 & 5.850 \\
\hline rural insurance (assisted) & 149 & 3.715 & 2.782 & 0.031 & 14.472 \\
\hline urban insurance (assisted) & 149 & 1.329 & 1.211 & 0.010 & 5.808 \\
\hline age65 & 149 & .089 & .0174 & .0482 & .132 \\
\hline beds & 149 & 3.979 & .736 & 2.568 & 6.06 \\
\hline bhi & 149 & 19.062 & 12.001 & 7.359 & 64.057 \\
\hline urban disposable income & 149 & 20.040 & 6.145 & 11.929 & 43.851 \\
\hline rural disposable income & 149 & 7.259 & 3.216 & 2.980 & 19.595 \\
\hline urbmi & 149 & 15.598 & 12.220 & 2.864 & 89.970 \\
\hline
\end{tabular}

a larger manufacturing sector and more generous insurance coverage are likely to demand more mental health care according to the literature.

People residing in a more economically developed and better educated region are more likely to be informed about mental diseases. A province with a larger manufacturing sector is more likely to have more workers working in a mass production process and suffer mental diseases (i.e. migrant workers). People residing in a province with better insurance plans will be covered for the fees charged by the mental hospitals.

All variables are weighted by population and the description of variables is shown in the following table 13. Note that inpatient volume for MCA hospitals is calculated by the average number of occupied beds per day ${ }^{*} 365$. We use the average length of stay of 45 days for all mental health providers (including MCA and MOH hospitals) in 2010 to calculate the inpatient volume in MCA hospitals (Ma, et al, 2014). The descriptive statistics are shown in table 14. 
Table 13: Descriptions of variables

\begin{tabular}{|l|l|}
\hline Variable name & Variable description \\
\hline moh inpatients & $\begin{array}{l}\text { number of inpatient visits in MOH hospital (per million } \\
\text { people) }\end{array}$ \\
\hline mca inpatient & $\begin{array}{l}\text { number of inpatient visits in MCA hospital (per million } \\
\text { people) }\end{array}$ \\
\hline moh beds & $\begin{array}{l}\text { Bed for patients with mental diseases in MOH hospitals } \\
\text { (per million people) }\end{array}$ \\
\hline mca beds & $\begin{array}{l}\text { Bed for patients with mental diseases in MOH hospitals } \\
\text { (per million people) }\end{array}$ \\
\hline education ratio & $\begin{array}{l}\text { Education(share of people with tertiary education or } \\
\text { above) }\end{array}$ \\
\hline industry ratio & $\begin{array}{l}\text { Industry (The share of manufacturing sector in total em- } \\
\text { ployment) }\end{array}$ \\
\hline disposable income & Average Disposable income in the province (1,000 RMB) \\
\hline urban insurance & coverage for urban social health insurance(\%) \\
\hline moh doctors & no. of doctors in a 1,000 people \\
\hline mca staffs & $\begin{array}{l}\text { the number of staffs working in MCA mental hospital } \\
\text { per million residents }\end{array}$ \\
\hline
\end{tabular}

Table 14: Summary of variables

\begin{tabular}{|l|c|c|c|c|c|}
\hline & count & mean & sd & min & $\max$ \\
\hline mental inpatients & 212 & 818.568 & 419.157 & 119.354 & 2029.798 \\
\hline mental beds & 212 & 161.715 & 113.132 & 7.372 & 596.239 \\
\hline mca inpatients & 200 & 276.238 & 190.537 & 0.0603 & 1213.583 \\
\hline mca mental beds & 200 & 50.240 & 34.047 & 6.639 & 260.996 \\
\hline education ratio & 212 & 8.036 & 6.259 & 0.024 & 37.350 \\
\hline urban insurance & 212 & 63.220 & 23.582 & 20.915 & 191.835 \\
\hline industry ratio & 212 & 20.835 & 12.706 & 0.0355 & 50.549 \\
\hline urban disposable income & 212 & 18.909 & 6.468 & 10.012 & 43.851 \\
\hline mca staff & 200 & 18.511 & 9.702 & 1.625 & 51.399 \\
\hline moh doctors & 212 & 1.662 & 0.523 & 0.792 & 3.646 \\
\hline
\end{tabular}

